Supporting Information for

\title{
Electronic Nature of Ketone Directing group as a Key to Control C-2 vs C-4 Alkenylation of Indoles
}

Veeranjaneyulu Lanke, Kiran R. Bettadapur, and Kandikere Ramaiah Prabhu*

Department of Organic Chemistry, Indian Institute of Science, Bangalore 560 012,

Karnataka, India 


\section{Contents}

1. General experimental SI-3

2. NMR Yield Calculation SI-3

3. Synthesis of Indole Derivatives SI-4

4. Optimization Studies Table SI-6

5. Typical experimental procedure SI-7

6. Deuterium Incorporation studies SI-8

7. Characterization data SI-9

8. Computational method SI-23

9. Table of Energies SI-25

10. Coordinates for structures SI-25

11. ${ }^{1} \mathrm{H}$ and ${ }^{13} \mathrm{C}$ NMR Spectra SI-30

12. Crystal Structure SI-88 


\section{General experimental}

NMR spectra were recorded on a 400 and $800 \mathrm{MHz}$ spectrometer in $\mathrm{CDCl}_{3}$, Tetramethylsilane (TMS; $\delta=0.00 \mathrm{ppm}$ ) served as an internal standard for $1 \mathrm{H}$ NMR. The corresponding residual nondeuterated solvent signal $(\mathrm{CDCl} ; \delta=77.00 \mathrm{ppm}$ ) was used as internal standard for 13C NMR. IR spectra were measured using a FT-IR spectrometer. Mass spectra were obtained with a Q-TOF Mass Spectrometer (ESI-HRMS). Flash column chromatography was carried out by packing glass columns with commercial silica gel 230-400 mesh (commercial suppliers) and thin-layer chromatography was carried out using silica gel GF-254. All catalysts, reagents and reactants were procured from commercial suppliers. Dichloroethane solvent was distilled over calcium hydride and stored over molecular sieves and used for all procedures. Other solvents, used for work up and chromatographic procedures were purchased from commercial suppliers and used without any further purification.

Note: In order to obtain the quartet for $\mathrm{COCF}_{3}$ in ${ }^{13} \mathrm{C}$ NMR, we have recorded the NMR a majority of the fluorinated compounds in an $800 \mathrm{MHz}$ spectrometer.

NMR Yield calculation - After work up, 0.5 equiv of terephthaldehyde was added into the round bottom flask containing the combined organic layers from the work up. Either an aliquot from the round bottom flask was taken, evaporated and then submitted for NMR, or the organic layer was completely evaporated and the residue was dissolved in an appropriate amount of solvent to submit for NMR. 


\section{Synthesis of Indole Derivatives}

i) Procedure for $\mathbf{N}$-methyl indole derivatives: ${ }^{1 \mathrm{a}}$

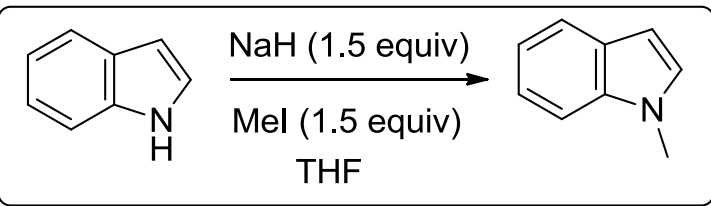

To a well-stirred solution of indole derivative $\left(10 \mathrm{mmol}, 1\right.$ equiv) in THF $(15 \mathrm{~mL})$ at $0{ }^{\circ} \mathrm{C}$ was added sodium hydride (60\% in mineral oil, $15 \mathrm{mmol}, 1.5$ equiv). The reaction was warmed to room temperature and allowed to stir for $30 \mathrm{~min}$. After $30 \mathrm{~min}$, the reaction flask was cooled to $0{ }^{\circ} \mathrm{C}$ again and methyl iodide (12 mmol, 1.2 equiv) was added drop-wise. The reaction mixture was warmed to room temperature and allowed to stir until the reaction completed (monitored by TLC) and then cooled to $0{ }^{\circ} \mathrm{C}$ and quenched with saturated $\mathrm{NH}_{4} \mathrm{Cl}$. The product was extracted with ether $(3 \times 20 \mathrm{~mL})$ and dried over $\mathrm{Na}_{2} \mathrm{SO}_{4}$. The organic phase was concentrated in vacuum to obtain the pure compound as a brown oil.

ii) 2,2,2-Trifluoro-1-(1-methyl-1H-indol-3-yl)ethan-1-one derivatives:

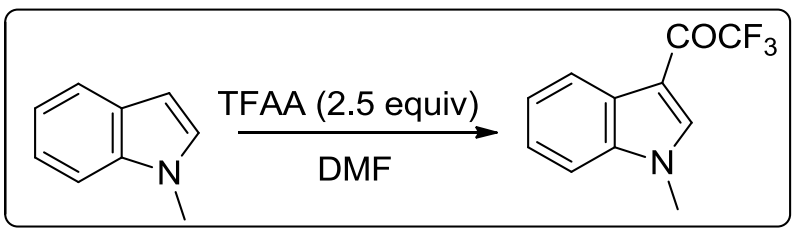

All the starting materials were prepared by following the reported method. ${ }^{1 \mathrm{~b}} \mathrm{~N}$-Methyl indole derivative (5 mmol, 1 equiv) was dissolved in a $10 \mathrm{~mL} \mathrm{DMF}$ and trifluoroacetic anhydride (TFAA, 25 mmol, 2.5 equiv) were added drop-wise. The reaction was monitored by TLC, after completion of the reaction the product was extracted by diethyl ether and water work-up to furnish the pure compound as a pale pink solid in good yields. 
iii) Procedure for 3-acetyl indole derivatives: ${ }^{2}$

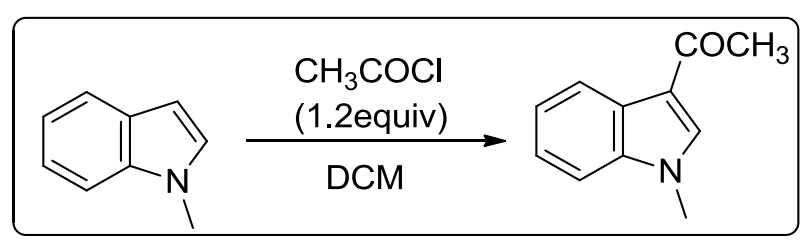

To a solution of indole derivative $(10 \mathrm{mmol}, 1$ equiv) in DCM $(10 \mathrm{~mL})$, was added $(15 \mathrm{mmol}$, 1.5 equiv) of $\mathrm{Et}_{2} \mathrm{AlCl}\left(0.98 \mathrm{~mol} / \mathrm{L}\right.$ in hexane) at $0{ }^{\circ} \mathrm{C}$. The mixture was stirred at $0{ }^{\circ} \mathrm{C}$ for 30 min. To this solution was added drop-wise a DCM solution $(10 \mathrm{~mL})$ of acetyl chloride (12 mmol, 1.2 equiv) at $0{ }^{\circ} \mathrm{C}$. The resulting solution was stirred at r.t until completion of the reaction (monitored by TLC), and aq. $\mathrm{NH}_{4} \mathrm{Cl}$ solution was added to quench the reaction. After the usual workup, the crude product was purified by column chromatography to obtain a white solid.

\section{Or}

To a cold solution $\left(0{ }^{\circ} \mathrm{C}\right)$ of indole derivative $(5 \mathrm{mmol}$, 1equiv) in anhyd DCE $(10 \mathrm{~mL})$ in a round-bottom flask, under nitrogen, was added a solution of acyl chloride (6 mmol, 1.2 equiv) in dry DCE $(10 \mathrm{~mL})$ via a syringe. Zirconium tetrachloride $(7.5 \mathrm{mmol}, 1.5$ equiv) was added under a flow of nitrogen. The reaction temperature was then gradually increased to $30{ }^{\circ} \mathrm{C}$. After completion of the reaction as indicated by TLC, the resultant mixture was quenched with water $(5 \mathrm{~mL})$ and extracted with EtOAc $(2 \times 15 \mathrm{~mL})$. The combined organic layer was washed with water $(10 \mathrm{~mL})$, dried with anhyd $\mathrm{Na}_{2} \mathrm{SO}_{4}$, and concentrated under vacuum. The crude mass was column purified on silica gel, with EtOAc/petroleum ether to provide the white solid product. 


\section{Optimization studies for C4-alkenylation of indole:}

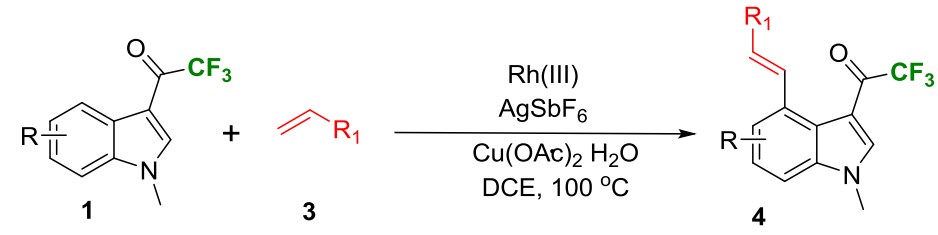

\begin{tabular}{|c|c|c|c|c|c|c|c|}
\hline $\mathrm{CF}_{3}$ - Ketone & $\begin{array}{l}\text { Methyl } \\
\text { Acrylate }\end{array}$ & $\begin{array}{l}\mathrm{Ru} \\
(\mathrm{mol} \%)\end{array}$ & $\begin{array}{l}\mathrm{Ag} \\
(\mathrm{mol} \%)\end{array}$ & $\begin{array}{l}\text { Oxidant } \\
\text { (equiv) }\end{array}$ & $\begin{array}{l}\text { Additive } \\
\text { (equiv) }\end{array}$ & $\begin{array}{l}\text { Product } \\
(\%)\end{array}$ & $\begin{array}{l}\text { SM } \\
(\%)\end{array}$ \\
\hline \multirow[t]{20}{*}{ lequiv } & 4 & 5 & 20 & 1 & - & 40 & 38 \\
\hline & 4 & 10 & 20 & 1 & - & 66 & 23 \\
\hline & 2 & 5 & 20 & 1 & - & 35 & 30 \\
\hline & 6 & 5 & 20 & 1 & - & 64 & 21 \\
\hline & 4 & 5 & 20 & 2 & - & 51 & 17 \\
\hline & 4 & 5 & 20 & 0.5 & & 45 & 39 \\
\hline & 6 & 10 & 20 & 1 & - & 65 & 21 \\
\hline & 4 & 5 & 20 & 1 & $\mathrm{AcOH} / 10$ & 31 & 36 \\
\hline & 4 & 5 & 20 & 1 & $\mathrm{MesCO}_{2} \mathrm{H} / 0.3$ & 20 & 68 \\
\hline & 4 & 5 & 20 & 1 & $\mathrm{AdmCO}_{2} \mathrm{H} / 0.3$ & 54 & 23 \\
\hline & 4 & 10 & 20 & 1 & $\mathrm{O}_{2}$ & 67 & 25 \\
\hline & 4 & 10 & 20 & 2 & $\mathrm{MesCO}_{2} \mathrm{H} / 0.3$ & 66 & 18 \\
\hline & 4 & 10 & 20 & 2 & $\mathrm{AdmCO}_{2} \mathrm{H} / 0.3$ & 64 & 23 \\
\hline & 4 & 10 & 40 & 1.5 & - & 54 & 17 \\
\hline & 4 & 10 & 20 & 1 & argon & 53 & 27 \\
\hline & 4 & $\operatorname{Rh}(10)$ & 20 & 1 & & 83 & 00 \\
\hline & 4 & $\operatorname{Rh}(5)$ & 10 & 1 & & 87 & 00 \\
\hline & 4 & $\operatorname{Rh}(2.5)$ & 7.5 & 1 & & 71 & 23 \\
\hline & 4 & $\operatorname{Rh}(2.5)$ & 10 & 1 & & $90(85)$ & $\mathbf{0 0}$ \\
\hline & 2 & $\operatorname{Rh}(2.5)$ & 10 & 1 & & 85 & 00 \\
\hline N-Bn CF CF$_{3}$ ketone & 4 & 10 & 20 & 1 & - & 60 & 32 \\
\hline
\end{tabular}

\section{Optimization studies for C2-alkenylation of indole:}<smiles>[R]C=C</smiles>
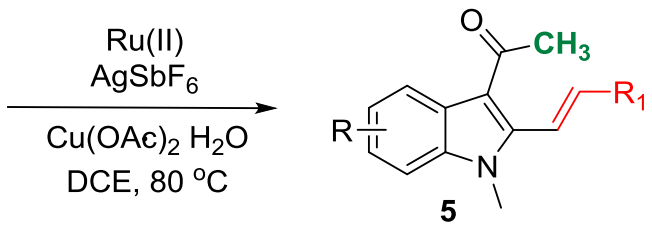

\begin{tabular}{|l|l|l|l|l|l|l|l|}
\hline $\begin{array}{l}\mathrm{CH}_{3^{-}} \\
\text {Ketone }\end{array}$ & $\begin{array}{l}\text { Methyl crylate } \\
\text { (equiv) }\end{array}$ & $\begin{array}{l}\mathrm{Ru} \\
(\mathrm{mol} \%)\end{array}$ & $\begin{array}{l}\mathrm{Ag} \\
(\mathrm{mol} \%)\end{array}$ & $\begin{array}{l}\text { Oxidant } \\
\text { (equiv) }\end{array}$ & $\begin{array}{l}\text { Additive } \\
\text { (equiv) }\end{array}$ & $\begin{array}{l}\text { Product } \\
(\%)\end{array}$ & $\begin{array}{l}\text { SM } \\
(\%)\end{array}$ \\
\hline 1equiv & 2 & 5 & 20 & 1 & - & 52 & 0 \\
\hline & 4 & 5 & 20 & 1 & - & 50 & 0 \\
\hline & 2 & 10 & 20 & 1 & - & 46 & 0 \\
\hline & 2 & 10 & 20 & 2 & - & 48 & 0 \\
\hline & 2 & 5 & 20 & 1 & $\mathrm{AcOH} / 10$ & 72 & 0 \\
\hline & $\mathbf{2}$ & $\mathbf{5}$ & $\mathbf{2 0}$ & $\mathbf{1}$ & $\mathbf{A c O H}(\mathbf{0 . 5 m l})$ & $\mathbf{8 2}$ & $\mathbf{0}$ \\
\hline & 2 & 5 & 20 & 1 & $\mathrm{AcOH}(1 \mathrm{ml})$ & 76 & 0 \\
\hline & 2 & 10 & 20 & 1 & $\mathrm{AcOH}(\mathrm{solvent})$ & 62 & 0 \\
\hline & 2 & $\mathrm{Rh}$ & 20 & 1 & - & 15 & 0 \\
\hline & 4 & $\mathrm{Rh}$ & 20 & 2 & $\mathrm{AcOH}(0.5 \mathrm{ml})$ & 20 & 0 \\
\hline
\end{tabular}




\section{Typical experimental procedure for the $\mathbf{C 4}$-alkenylation of indoles}

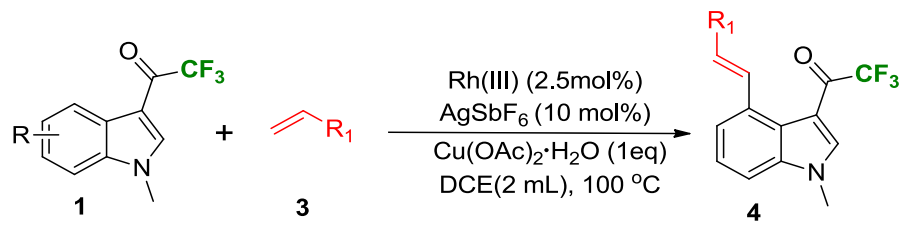

To a pre-dried $8 \mathrm{~mL}$ screw cap vial was added 1-methyl-3-(trifluoroacetyl)-1H-indole derivative (0.3 mmol, 1 equiv), $\left[\mathrm{RhCp}^{*} \mathrm{Cl}_{2}\right]_{2}(2.5 \mathrm{~mol} \%), \mathrm{AgSbF}_{6}(10 \mathrm{~mol} \%)$, acrylate derivative (1.2 mmol, 4 equiv) and $\mathrm{Cu}(\mathrm{OAc})_{2} \cdot \mathrm{H}_{2} \mathrm{O}(0.3 \mathrm{mmol}$, 1equiv) and dichloroethane (2 $\mathrm{mL})$. The vial was placed in a pre-heated $\left(100^{\circ} \mathrm{C}\right)$ metal block. After $6-8 \mathrm{~h}$ (completion of reaction monitored by TLC), the reaction mixture was cooled to room temperature, and diluted with diethyl ether and passed through a short silica gel (100-200 mesh size) bed, and repeatedly washed with diethyl ether (20 mL x 3 times). The combined organic layers were concentrated under reduced pressure and the crude product was purified on a silica gel column using EtOAc/hexane mixture (as indicated by the $R_{f}$ value for the corresponding products).

\section{Typical experimental procedure for the $\mathbf{C} 2$-alkenylation of indoles}
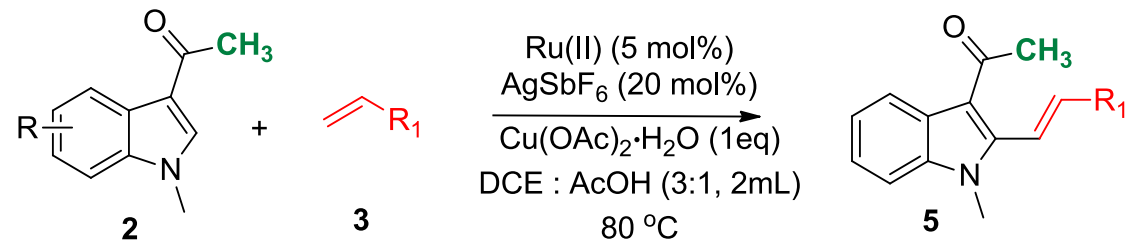

To a pre-dried $8 \mathrm{~mL}$ screw cap vial was added 3 -acetyl indole derivative ( $0.3 \mathrm{mmol}, 1$ equiv), $\left[\mathrm{Ru}(\text { p-cymene }) \mathrm{Cl}_{2}\right]_{2}(5 \mathrm{~mol} \%), \mathrm{AgSbF}_{6}(20 \mathrm{~mol} \%)$, acrylate derivative (0.6 mmol, 2 equiv) and $\mathrm{Cu}(\mathrm{OAc})_{2} \cdot \mathrm{H}_{2} \mathrm{O}(0.3 \mathrm{mmol}$, lequiv). To this reaction mixture, acetic acid $(0.5 \mathrm{~mL})$ and dichloroethane $(1.5 \mathrm{~mL})$ was added and the vial was placed in a pre-heated $\left(80^{\circ} \mathrm{C}\right)$ metal block. After 2-5h (completion of reaction monitored by TLC), the reaction mixture was cooled to room temperature, and diluted with diethyl ether and passed through a short silica gel (100200 mesh size) bed, and repeatedly washed with diethyl ether ( $20 \mathrm{~mL}$ x 3 times). The combined organic layers were concentrated under reduced pressure and the crude product was purified on a silica gel column using EtOAc/hexane mixture (as indicated by the $R_{f}$ value for the corresponding products). 


\section{Deuterium incorporation studies:}

i) To a pre-dried $8 \mathrm{~mL}$ screw cap vial was added 1methyl-3-(trifluoroacetyl)-1H-indole derivative (0.2mmol, 1 equiv), $\left[\mathrm{RhCp}^{*} \mathrm{Cl}_{2}\right]_{2}(2.5 \mathrm{~mol} \%), \mathrm{AgSbF}_{6}$ (10mol \%), and $\mathrm{Cu}(\mathrm{OAc})_{2} \cdot \mathrm{H}_{2} \mathrm{O}$ (0.2mmol, 1equiv), $\mathrm{CD}_{3} \mathrm{CO}_{2} \mathrm{D}$ (2 mmol,10 equiv) and dichloroethane (2 $\mathrm{mL})$. The vial was placed in a pre-heated $\left(100{ }^{\circ} \mathrm{C}\right)$ metal block. After $6 \mathrm{~h}$, the reaction mixture was cooled to room temperature, and diluted with diethyl ether and passed through a short silica gel (100-200 mesh size)

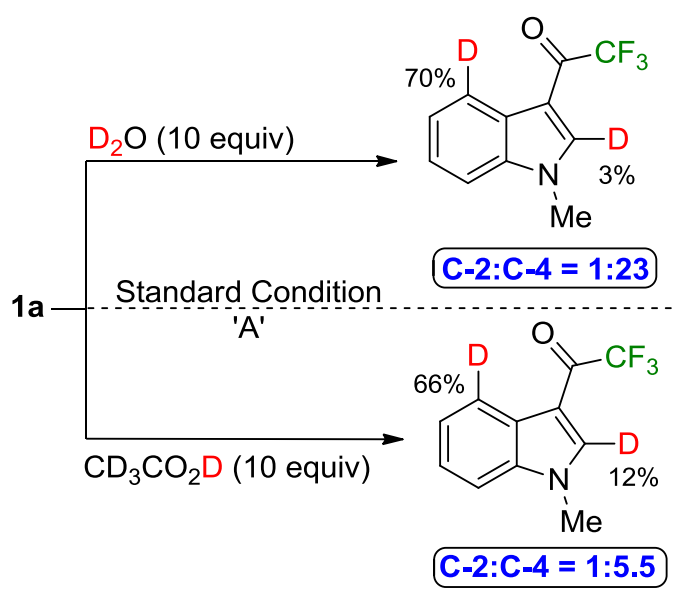
bed, and repeatedly washed with diethyl ether (20 mL x 3 times). The combined organic layers were concentrated under reduced pressure and the crude product was submitted for NMR.

\section{Note: For labeling with deuterium from $\mathrm{D}_{2} \mathrm{O}:-\mathrm{D}_{2} \mathrm{O}$ was used instead of $\mathrm{CD}_{3} \mathrm{COOD}$, and}

\section{$0.1 \mathrm{mmol}$ scale reaction was performed.}

ii) Deuterium incorporation experiment with 2a:

To a pre-dried $8 \mathrm{~mL}$ screw cap vial was added $N$ methyl-3-acetylindole $(0.2 \mathrm{mmol}, 1$ equiv), $[\mathrm{Ru}(p$ cymene $\left.\mathrm{Cl}_{2}\right]_{2}$ (5mol \%), $\mathrm{AgSbF}_{6}(20 \mathrm{~mol} \%)$, and $\mathrm{Cu}(\mathrm{OAc})_{2} \cdot \mathrm{H}_{2} \mathrm{O}(0.2 \mathrm{mmol}$, 1equiv). To this reaction mixture, deuterated acetic acid $(0.5 \mathrm{~mL})$ and dichloroethane $(1.5 \mathrm{~mL})$ was added and the vial was placed in a pre-heated $\left(80^{\circ} \mathrm{C}\right)$ metal block. After $6 \mathrm{~h}$, the reaction mixture was cooled to room temperature, and diluted with diethyl ether and passed through a

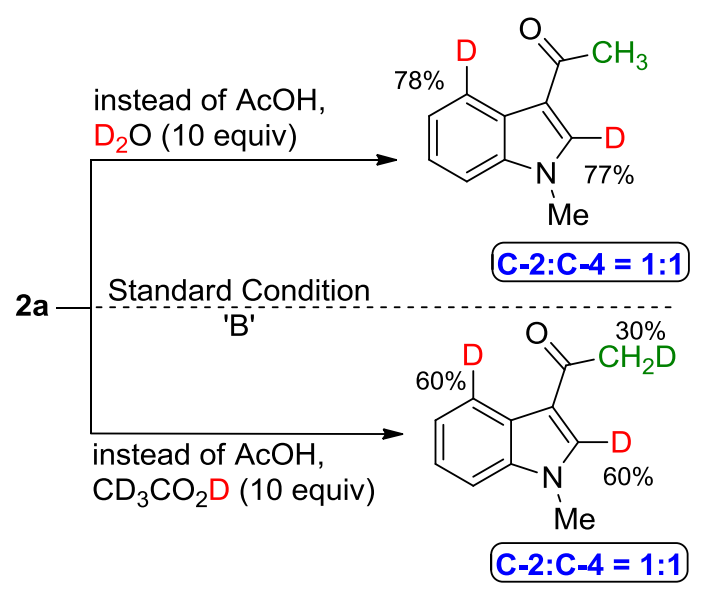
short silica gel (100-200 mesh size) bed, and repeatedly washed with diethyl ether (20 mL x 3 times). The combined organic layers were concentrated under reduced pressure and the crude product was submitted for NMR.

\section{Note: For labelling with deuterium from $\mathrm{D}_{2} \mathrm{O}: \mathrm{D}_{2} \mathrm{O}$ was used instead of $\mathrm{CD}_{3} \mathrm{COOD}$, and} $0.1 \mathrm{mmol}$ scale reaction was performed. 


\section{Characterization data for the products:}

1. Methyl (E)-3-(1-methyl-3-(2,2,2-trifluoroacetyl)-1H-indol-4-yl)acrylate(4a):

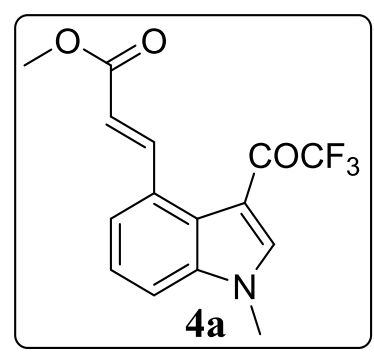

Prepared as shown in general procedure. Crude reaction mixture was purified on a flash column to obtain the product as yellow solid; Yield - $80 \mathrm{mg},(86 \%) ; \mathbf{m p}: 158-160{ }^{\circ} \mathrm{C} ; R f$ (20\% EtOAc/Hexane) 0.2; IR (KBr, cm $\left.{ }^{-1}\right): 1712,1528,{ }^{1} \mathbf{H ~ N M R ~}\left(800 \mathrm{MHz}, \mathrm{CDCl}_{3}\right): \delta 3.86$ $(\mathrm{s}, 3 \mathrm{H}) 3.94(\mathrm{~s}, 3 \mathrm{H}) 6.35(\mathrm{~d}, J=15.81 \mathrm{~Hz}, 1 \mathrm{H}) 7.40-7.44(\mathrm{~m}, 2 \mathrm{H}) 7.60(\mathrm{dd}, J=6.33,2.09$ $\mathrm{Hz}, 1 \mathrm{H}) 8.04(\mathrm{~S}, 1 \mathrm{H}) 9.18(\mathrm{~d}, J=15.81 \mathrm{~Hz}, 1 \mathrm{H}) ;{ }^{13} \mathbf{C ~ N M R}\left(200 \mathrm{MHz}, \mathrm{CDCl}_{3}\right): \delta 34.29$, $51.73,110.18,111.59,117.61(\mathrm{q}, J=293.4 \mathrm{~Hz}$,

$\left.\mathrm{CF}_{3}\right), 118.93,122.79,124.80,125.02,130.47,138.47,140.88(\mathrm{~d}, J=5.4$

$\mathrm{Hz}), 145.79,167.45,173.76\left(\mathrm{q}, J=34.8 \mathrm{~Hz}, \mathrm{COCF}_{3}\right)$; HRESI-MS $(\mathrm{m} / \mathrm{z})$ : Calculated for $\mathrm{C}_{15} \mathrm{H}_{12} \mathrm{~F}_{3} \mathrm{NO}_{3}(\mathrm{M}+\mathrm{Na}): 334.0667$, found $(\mathrm{M}+\mathrm{Na}): 334.0663$.

2. Methyl (E)-3-(6-bromo-1-methyl-3-(2,2,2-trifluoroacetyl)-1H-indol-4-yl)acrylate (4b):

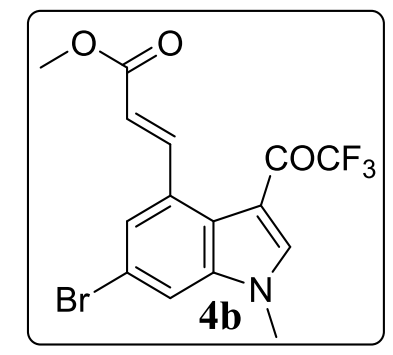

Prepared as shown in general procedure. Crude reaction mixture was purified on a flash column to obtain the product as Yellow solid; Yield - $82 \mathrm{mg}$, (72\%); $\mathbf{m p}: 168-171{ }^{\circ} \mathrm{C} ; R f(20 \%$ EtOAc/Hexane) 0.2; IR ( $\left.\mathrm{KBr}, \mathrm{cm}^{-1}\right): 1713,1669,1526,{ }^{1} \mathbf{H} \mathbf{~ N M R}\left(800 \mathrm{MHz}, \mathrm{CDCl}_{3}\right): \delta 3.85$ $(\mathrm{s}, 3 \mathrm{H}) 3.91(\mathrm{~s}, 3 \mathrm{H}) 6.32(\mathrm{~d}, J=15.78 \mathrm{~Hz}, 1 \mathrm{H}) 7.56(\mathrm{~d}, J=1.65 \mathrm{~Hz}, 1 \mathrm{H}) 7.66$ (d, J=1.30 Hz, 1 H) $8.00(\mathrm{~s}, 1 \mathrm{H}) 9.03(\mathrm{~d}, J=15.78 \mathrm{~Hz}, 1 \mathrm{H}) ;{ }^{13} \mathbf{C ~ N M R}\left(200 \mathrm{MHz}, \mathrm{CDCl}_{3}\right): \delta 34.49,51.96$, 110.20, 114.49, 117.42(q, $\left.J=293 \mathrm{~Hz}, \mathbf{C F}_{3}\right), 118.36,120.08,123.86,125.59,131.79,139.19$, 141.35, 144.41, 167.15, 173.74(q, $\left.J=35 \mathrm{~Hz}, \mathbf{C O C F}_{3}\right)$; HRESI-MS $(\mathrm{m} / \mathrm{z})$ : Calculated for $\mathrm{C}_{15} \mathrm{H}_{11} \mathrm{BrF}_{3} \mathrm{NO}_{3}(\mathrm{M}+\mathrm{Na}): 411.9772$, found $(\mathrm{M}+\mathrm{Na}): 411.9772$ 
3. Methyl (E)-3-(6-chloro-1-methyl-3-(2,2,2-trifluoroacetyl)-1H-indol-4-yl)acrylate (4c):

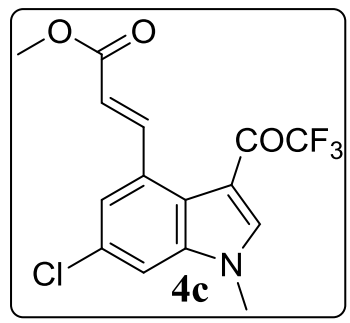

Prepared as shown in general procedure. Crude reaction mixture was purified on a flash column to obtain the product as Yellow solid; Yield - $76 \mathrm{mg},(74 \%) ; \mathbf{m p}: 142-145{ }^{\circ} \mathrm{C} ; R f(20 \%$ EtOAc/Hexane) 0.2; IR ( $\left.\mathrm{KBr}, \mathrm{cm}^{-1}\right): 1710,1652,1543,{ }^{1} \mathbf{H} \mathbf{N M R}\left(800 \mathrm{MHz}, \mathrm{CDCl}_{3}\right): \delta 3.82$ $(\mathrm{s}, 3 \mathrm{H}) 3.88(\mathrm{~s}, 3 \mathrm{H}) 6.26(\mathrm{~d}, J=15.77 \mathrm{~Hz}, 1 \mathrm{H}) 7.34(\mathrm{~d}, J=1.71 \mathrm{~Hz}, 1 \mathrm{H}) 7.41(\mathrm{~d}, J=1.59 \mathrm{~Hz}, 1$ H) $7.98(\mathrm{~s}, 1 \mathrm{H}) 8.96(\mathrm{~d}, J=15.77 \mathrm{~Hz}, 1 \mathrm{H}) ;{ }^{13} \mathbf{C} \mathbf{N M R}\left(200 \mathrm{MHz}, \mathrm{CDCl}_{3}\right)$ : $\delta$ 34.46, 51.92, 110.05, 115.56, 117.41 (q, $J=291.5 \mathrm{~Hz}, \mathrm{CF}_{3}$ ), 119.87, 122.76, 123.42, 130.79, 131.3, 138.95, $141.61(\mathrm{~d}, J=4.7 \mathrm{~Hz}), 144.43,167.17,173.69$ (q, J=34Hz, COCF $)$; HRESI-MS (m/z): Calculated for $\mathrm{C}_{15} \mathrm{H}_{11} \mathrm{ClF}_{3} \mathrm{NO}_{3}(\mathrm{M}+\mathrm{Na}): 368.0277$, found $(\mathrm{M}+\mathrm{Na}): 368.0275$

4. Methyl (E)-3-(5-methoxy-1-methyl-3-(2,2,2-trifluoroacetyl)-1H-indol-4-yl)acrylate (4d):

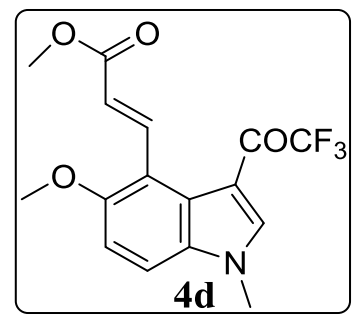

Prepared as shown in general procedure. Crude reaction mixture was purified on a flash column to obtain the product as Yellow solid; Yield - $72 \mathrm{mg}$, (70\%); mp: $149-152{ }^{\circ} \mathrm{C} ; R f(30 \%$ EtOAc/Hexane) 0.2; IR (KBr, cm $\left.{ }^{-1}\right): 1711,1532,{ }^{1} \mathbf{H} \mathbf{N M R}\left(400 \mathrm{MHz}, \mathrm{CDCl}_{3}\right): \delta 3.84(\mathrm{~s}, 3 \mathrm{H})$ $3.87(\mathrm{~s}, 3 \mathrm{H}) 3.91(\mathrm{~s}, 3 \mathrm{H}) 6.65(\mathrm{~d}, J=15.89 \mathrm{~Hz}, 1 \mathrm{H}) 7.06(\mathrm{~d}, J=9.05 \mathrm{~Hz}, 1 \mathrm{H}) 7.31$ (d, J=9.05 $\mathrm{Hz}, 1 \mathrm{H}) 7.94(\mathrm{~s}, 1 \mathrm{H}) 8.84(\mathrm{~d}, J=16.14 \mathrm{~Hz}, 1 \mathrm{H}) ;{ }^{13} \mathrm{C} \mathrm{NMR}\left(200 \mathrm{MHz}, \mathrm{CDCl}_{3}\right): \delta 34.15,51.46$, 56.4, 109.82, 109.98, 111.65, 117.28, 121.43, 127.02, 133.12, 140.73, 141.14, 141.19, 156.38, $168.51,173.27$

; HRESI-MS (m/z): Calculated for $\mathrm{C}_{16} \mathrm{H}_{14} \mathrm{~F}_{3} \mathrm{NO}_{4}(\mathrm{M}+\mathrm{Na})$ : 364.0773 , found $(\mathrm{M}+\mathrm{Na})$ : 364.0774 . 
5. Methyl (E)-3-(1-methyl-6-phenyl-3-(2,2,2-trifluoroacetyl)-1H-indol-4-yl)acrylate (4e):

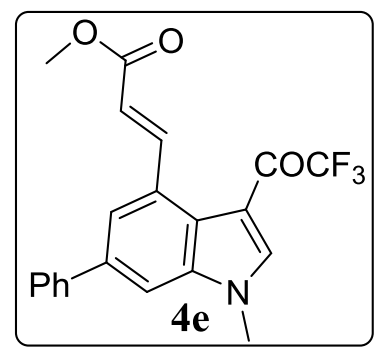

Prepared as shown in general procedure. Crude reaction mixture was purified on a flash column to obtain the product as Yellow solid; Yield - $98 \mathrm{mg},(85 \%) ; \mathbf{m p}: 184-187{ }^{\circ} \mathrm{C} ; R f(30 \%$ EtOAc/Hexane) 0.2; IR (KBr, cm $\left.{ }^{-1}\right): 1707,1673,1487,{ }^{1} \mathbf{H} \mathbf{N M R}\left(800 \mathrm{MHz}, \mathrm{CDCl}_{3}\right): \delta 3.86$ $(\mathrm{s}, 3 \mathrm{H}) 3.98(\mathrm{~s}, 3 \mathrm{H}) 6.39(\mathrm{~d}, J=15.71 \mathrm{~Hz}, 1 \mathrm{H}) 7.39-7.44(\mathrm{~m}, 1 \mathrm{H}) 7.50(\mathrm{t}, J=7.50 \mathrm{~Hz}, 2 \mathrm{H})$ $7.58(\mathrm{~s}, 1 \mathrm{H}) 7.64(\mathrm{~d}, J=7.57 \mathrm{~Hz}, 2 \mathrm{H}) 7.76(\mathrm{~s}, 1 \mathrm{H}) 8.06(\mathrm{~s}, 1 \mathrm{H}) 9.20(\mathrm{~d}, J=15.85 \mathrm{~Hz}, 1 \mathrm{H})$; ${ }^{13} \mathrm{C}$ NMR (200 MHz, $\mathrm{CDCl}_{3}$ ): $\delta$ 34.44, 51.86, 110.01, 117.62 (q, J=291Hz, $\mathbf{C F}_{3}$ ), 119.08, 122.2 , 124.13, 127.3, 127.85, 129.05, 130.47, 138.27, 139.17, 140.12, 141.57 (d, J=4.4Hz), 145.86, 165.51, 173.72 (q, $\left.J=34 \mathrm{~Hz}, \mathrm{COCF}_{3}\right)$; HRESI-MS $(\mathrm{m} / \mathrm{z})$ : Calculated for $\mathrm{C}_{21} \mathrm{H}_{16} \mathrm{~F}_{3} \mathrm{NO}_{3}$ $(\mathrm{M}+\mathrm{Na}): 410.0980$, found $(\mathrm{M}+\mathrm{Na}): 410.0982$.

6. Methyl (E)-3-(1,2-dimethyl-3-(2,2,2-trifluoroacetyl)-1H-indol-4-yl)acrylate (4f):

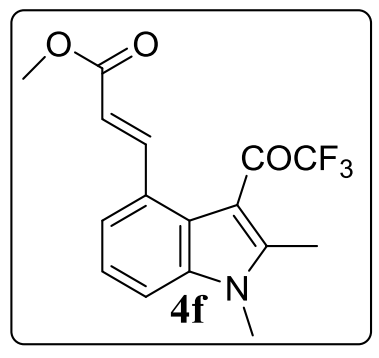

Prepared as shown in general procedure. Crude reaction mixture was purified on a flash column to obtain the product as Yellow solid; Yield - $96 \mathrm{mg}$, (97\%); mp: $102-104{ }^{\circ} \mathrm{C} ; R f(20 \%$ EtOAc/Hexane) 0.2; IR (KBr, cm $\left.{ }^{-1}\right): 1703,1652,1407,{ }^{1} \mathbf{H} \mathbf{~ N M R}\left(800 \mathrm{MHz}, \mathrm{CDCl}_{3}\right): \delta 2.57$ $(\mathrm{s}, 3 \mathrm{H}) 3.74(\mathrm{~s}, 3 \mathrm{H}) 3.81(\mathrm{~s}, 3 \mathrm{H}) 6.36(\mathrm{~d}, J=15.71 \mathrm{~Hz}, 1 \mathrm{H}) 7.29$ (t, J=7.78 Hz, $1 \mathrm{H}) 7.34$ (d, $J=8.13 \mathrm{~Hz}, 1 \mathrm{H}) 7.43(\mathrm{~d}, J=7.40 \mathrm{~Hz}, 1 \mathrm{H}) 8.07$ (d, $J=15.74 \mathrm{~Hz}, 1 \mathrm{H}) ;{ }^{13} \mathbf{C}$ NMR $(200 \mathrm{MHz}$, $\left.\mathrm{CDCl}_{3}\right): \delta 12.12,30.28,51.65,109.49,111.12,116.45\left(\mathrm{q}, J=292 \mathrm{~Hz}, \mathrm{CF}_{3}\right), 118.60,122.2$, 123.03, 124.71, 128.28, 137.54, 144.11, 145.73, 167.21, 180.65 (q, J=35Hz, COCF 3 ); HRESIMS $(m / z)$ : Calculated for $\mathrm{C}_{16} \mathrm{H}_{14} \mathrm{~F}_{3} \mathrm{NO}_{3}(\mathrm{M}+\mathrm{Na})$ : 348.0823 , found $(\mathrm{M}+\mathrm{Na})$ : 348.0877 . 
7. Methyl (E)-3-(1-methyl-2-phenyl-3-(2,2,2-trifluoroacetyl)-1H-indol-4-yl)acrylate (4g):

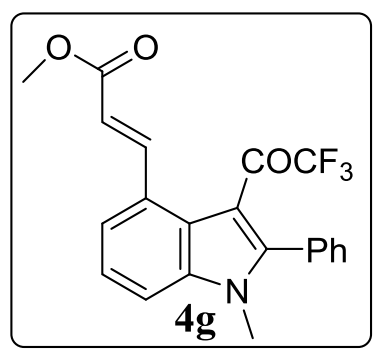

Prepared as shown in general procedure. Crude reaction mixture was purified on a flash column to obtain the product as Yellow solid; Yield - $96 \mathrm{mg},(84 \%)$; $\mathbf{m p}: 179-182{ }^{\circ} \mathrm{C} ; R f(20 \%$ EtOAc/Hexane) 0.2; IR (KBr, cm $\left.{ }^{-1}\right): 1707,1673,1487,{ }^{1} \mathbf{H} \mathbf{N M R}\left(400 \mathrm{MHz}, \mathrm{CDCl}_{3}\right): \delta 3.61$ $(\mathrm{s}, 3 \mathrm{H}) 3.81(\mathrm{~s}, 3 \mathrm{H}) 6.35(\mathrm{~d}, J=15.65 \mathrm{~Hz}, 1 \mathrm{H}) 7.33$ - $7.42(\mathrm{~m}, 3 \mathrm{H}) 7.42$ - $7.47(\mathrm{~m}, 1 \mathrm{H}) 7.47$ - $7.58(\mathrm{~m}, 4 \mathrm{H}) 8.21(\mathrm{~d}, J=15.65 \mathrm{~Hz}, 1 \mathrm{H}) ;{ }^{13} \mathbf{C ~ N M R}\left(200 \mathrm{MHz}, \mathrm{CDCl}_{3}\right): \delta 31.3,51.58,110.1$, 114.33 , 116.62, 119.14, 122.19, 123.86, 124.58, 128.36, 128.72, 129.28, 129.98, 130.46, 137.56, 143.47, 147.07, 167.05, 181.05 (d, $\left.J=33 \mathrm{~Hz}, \mathbf{C O C F}_{3}\right)$; HRESI-MS (m/z): Calculated for $\mathrm{C}_{21} \mathrm{H}_{16} \mathrm{~F}_{3} \mathrm{NO}_{3}(\mathrm{M}+\mathrm{Na}): 410.0980$, found $(\mathrm{M}+\mathrm{Na}): 410.0985$.

8. Methyl (E)-3-(1-benzyl-3-(2,2,2-trifluoroacetyl)-1H-indol-4-yl)acrylate (4h):

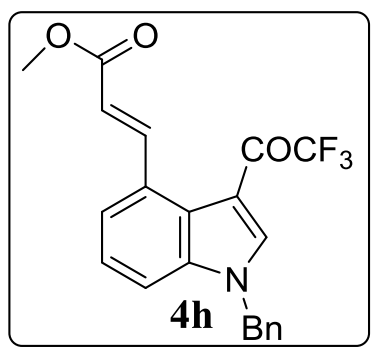

Prepared as shown in general procedure. Crude reaction mixture was purified on a flash column to obtain the product as Yellow solid; Yield - $109 \mathrm{mg}$, (95\%); mp: $153-156{ }^{\circ} \mathrm{C} ; R f(30 \%$ EtOAc/Hexane) 0.2; IR (KBr, cm $\left.{ }^{-1}\right): 1707,1673,1487,{ }^{1} \mathbf{H} \mathbf{~ N M R}\left(800 \mathrm{MHz} \mathrm{CDCl}_{3}\right): \delta 3.84$ (s, $3 \mathrm{H}) 5.43(\mathrm{~s}, 2 \mathrm{H}) 6.33(\mathrm{~d}, J=15.71 \mathrm{~Hz}, 1 \mathrm{H}) 7.15-7.18(\mathrm{~m}, 2 \mathrm{H}) 7.29-7.38(\mathrm{~m}, 5 \mathrm{H}) 7.54$ $(\mathrm{d}, J=7.43 \mathrm{~Hz}, 1 \mathrm{H}) 8.11(\mathrm{~s}, 1 \mathrm{H}) 9.17(\mathrm{~d}, J=15.85 \mathrm{~Hz}, 1 \mathrm{H}) ;{ }^{13} \mathbf{C} \mathbf{N M R}\left(200 \mathrm{MHz}, \mathrm{CDCl}_{3}\right): \delta$ 51.6, 51.86, 110.6, 112.41, 117.57 (q, $J=293 \mathrm{~Hz}, \mathbf{C F}_{3}$ ), 118.98, 122.97, 124.98, 125.28, 126.94, $128.71,129.31,130.59,134.35,137.95,140.48$ (d, J=4.8Hz), 145.91, 167.55, 174.0 (q, $\left.J=35 \mathrm{~Hz}, \mathbf{C O C F}_{3}\right)$; HRESI-MS $(\mathrm{m} / \mathrm{z})$ : Calculated for $\mathrm{C}_{21} \mathrm{H}_{16} \mathrm{~F}_{3} \mathrm{NO}_{3}(\mathrm{M}+\mathrm{Na}): 410.0980$, found $(\mathrm{M}+\mathrm{Na}): 410.0981$. 
9. Ethyl (E)-3-(1-methyl-3-(2,2,2-trifluoroacetyl)-1H-indol-4-yl)acrylate (4i):

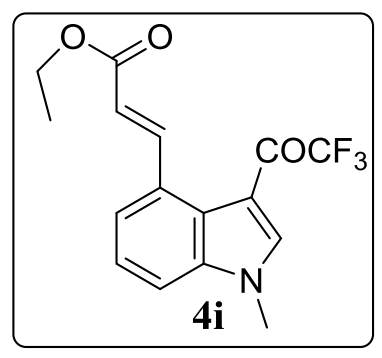

Prepared as shown in general procedure. Crude reaction mixture was purified on a flash column to obtain the product as Yellow solid; Yield - $83 \mathrm{mg},(88 \%) ; \mathbf{m p}: 156-158{ }^{\circ} \mathrm{C} ; R f(20 \%$ EtOAc/Hexane) 0.2; IR (KBr, cm $\left.{ }^{-1}\right): 1717,1608,{ }^{1} \mathbf{H} \mathbf{N M R}\left(800 \mathrm{MHz}, \mathrm{CDCl}_{3}\right): \delta 1.37(\mathrm{t}, J=7.15$ $\mathrm{Hz}, 3 \mathrm{H}) 3.92$ (s, $3 \mathrm{H}) 4.30$ (q, J=7.15 Hz, 2 H) 6.33 (d, J=15.71 Hz, $1 \mathrm{H}) 7.36-7.43$ (m, $2 \mathrm{H}) 7.56$ $(\mathrm{d}, J=7.01 \mathrm{~Hz}, 1 \mathrm{H}) 8.02(\mathrm{~s}, 1 \mathrm{H}) 9.17(\mathrm{~d}, J=15.85 \mathrm{~Hz}, 1 \mathrm{H}) ;{ }^{13} \mathbf{C} \mathbf{N M R}\left(200 \mathrm{MHz}, \mathrm{CDCl}_{3}\right): \delta 14.4$, 34.38, 60.51, 110.12, 111.68, 117.6 (q, $J=292 \mathrm{~Hz}, \mathrm{CF}_{3}$ ), 119.2, 122.72, 124.81, 125.03, 130.38, 138.48, $141.01(\mathrm{~d}, J=5.0 \mathrm{~Hz}), 145.7,167.15,173.71\left(\mathrm{q}, J=35 \mathrm{~Hz}, \mathbf{C O C F}_{3}\right)$; HRESI-MS $(\mathrm{m} / \mathrm{z})$ : Calculated for $\mathrm{C}_{16} \mathrm{H}_{14} \mathrm{~F}_{3} \mathrm{NO}_{3}(\mathrm{M}+\mathrm{Na})$ : 348.0823, found $(\mathrm{M}+\mathrm{Na}): 348.0826$.

10. Butyl (E)-3-(1-methyl-3-(2,2,2-trifluoroacetyl)-1H-indol-4-yl)acrylate (4j):

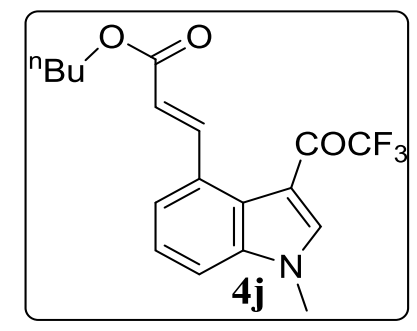

Prepared as shown in general procedure. Crude reaction mixture was purified on a flash column to obtain the product as Yellow solid; Yield - $92 \mathrm{mg},(87 \%) ; \mathbf{m p}: 117-121{ }^{\circ} \mathrm{C} ; R f(20 \%$ EtOAc/Hexane) 0.2; IR (KBr, cm $\left.{ }^{-1}\right): 1706,1669,{ }^{1} \mathbf{H} \mathbf{N M R}\left(800 \mathrm{MHz}, \mathrm{CDCl}_{3}\right): \delta 0.98(\mathrm{t}, J=7.43$ Hz, 3 H) 1.49 (sxt, J=7.49 Hz, 2 H) 1.70 - $1.74(\mathrm{~m}, 2 \mathrm{H}) 3.91(\mathrm{~s}, 3 \mathrm{H}) 4.24$ (t, J=6.66 Hz, $2 \mathrm{H}) 6.32$ $(\mathrm{d}, J=15.71 \mathrm{~Hz}, 1 \mathrm{H}) 7.36(\mathrm{t}, J=7.78 \mathrm{~Hz}, 1 \mathrm{H}) 7.39(\mathrm{~d}, J=7.85 \mathrm{~Hz}, 1 \mathrm{H}) 7.54(\mathrm{~d}, J=7.29 \mathrm{~Hz}, 1 \mathrm{H})$ $8.00(\mathrm{~s}, 1 \mathrm{H}) 9.15(\mathrm{~d}, J=15.85 \mathrm{~Hz}, 1 \mathrm{H}) ;{ }^{13} \mathbf{C} \mathbf{N M R}\left(200 \mathrm{MHz}, \mathrm{CDCl}_{3}\right): \delta 13.82,19.23,30.83$, 34.34, 64.4, 110.05, 111.74, 117.59 (q, $J=292 \mathrm{~Hz}, \mathrm{CF}_{3}$ ), 119.07, 122.61, 124.76, 125.0, 130.23, 138.47, 145.02 (d, $J=4.4 \mathrm{~Hz}), 167.24,173.67$ (q, $\left.J=34 \mathrm{~Hz}, \mathbf{C O C F}_{3}\right)$; HRESI-MS ( $\mathrm{m} / \mathrm{z}$ ): Calculated for $\mathrm{C}_{18} \mathrm{H}_{18} \mathrm{~F}_{3} \mathrm{NO}_{3}(\mathrm{M}+\mathrm{Na}): 376.1136$, found $(\mathrm{M}+\mathrm{Na}): 376.1133$ 


\section{Dimethyl (E)-(2-(1-methyl-3-(2,2,2-trifluoroacetyl)-1H-indol-4-yl)vinyl)phosphonate} $(4 k)$ :

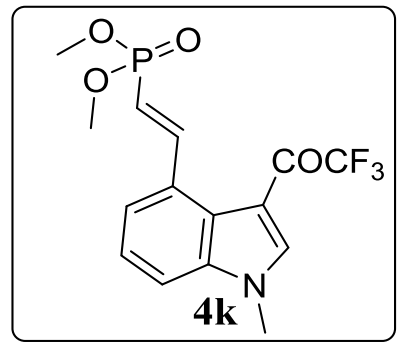

Prepared as shown in general procedure. Crude reaction mixture was purified on a flash column to obtain the product as Orange solid; Yield - $34 \mathrm{mg},(32 \%)$; $\boldsymbol{m p}: 156-159{ }^{\circ} \mathrm{C} ; R f(100 \% \mathrm{EtOAc})$ 0.4; IR $\left(\mathrm{KBr}, \mathrm{cm}^{-1}\right): 3375,1673,1444,{ }^{1} \mathbf{H} \mathbf{N M R}\left(800 \mathrm{MHz} \mathrm{CDCl}_{3}\right): \delta 3.87$ (s, $\left.3 \mathrm{H}\right) 3.88(\mathrm{~s}, 3 \mathrm{H})$ $3.94(\mathrm{~s}, 3 \mathrm{H}) 6.15(\mathrm{dd}, J=19.92,17.39 \mathrm{~Hz}, 1 \mathrm{H}) 7.41(\mathrm{t}, J=7.71 \mathrm{~Hz}, 1 \mathrm{H}) 7.44$ (dd, $J=8.06,0.91 \mathrm{~Hz}$, $1 \mathrm{H}) 7.56(\mathrm{~d}, J=7.29 \mathrm{~Hz}, 1 \mathrm{H}) 8.04(\mathrm{~d}, J=1.54 \mathrm{~Hz}, 1 \mathrm{H}) 8.88(\mathrm{dd}, J=22.02,17.34 \mathrm{~Hz}, 1 \mathrm{H}) ;{ }^{13} \mathrm{C}$ NMR (200 MHz, $\left.\mathrm{CDCl}_{3}\right): \delta 34.4,52.87,52.89,110.03,111.82,113.44$ (d, J=191Hz), 117.55 (q, $\left.J=292 \mathrm{~Hz}, \mathrm{CF}_{3}\right), 122.6,124.65,124.87,130.84$ (d, $\left.J=24 \mathrm{~Hz}\right), 138.45,141.06$ (d, J=4.5Hz), 149.72 $(\mathrm{d}, J=8.2 \mathrm{~Hz}), 173.74$ (q, $\left.J=33 \mathrm{~Hz}, \mathbf{C O C F}_{3}\right)$; HRESI-MS $(\mathrm{m} / \mathrm{z})$ : Calculated for $\mathrm{C}_{15} \mathrm{H}_{15} \mathrm{PF}_{3} \mathrm{NO}_{4}(\mathrm{M}$ $+\mathrm{Na}): 384.0588$, found $(\mathrm{M}+\mathrm{Na}): 384.0589$

\section{2. (E)-1-(4-(4-chlorostyryl)-1-methyl-1H-indol-3-yl)-2,2,2-trifluoroethan-1-one (4l):}

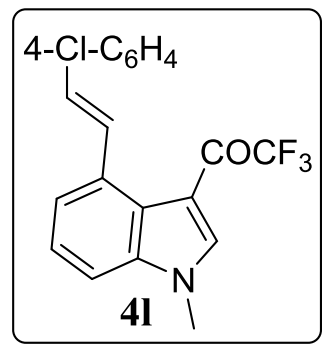

Prepared as shown in general procedure. Crude reaction mixture was purified on a flash column to obtain the product as Yellow solid; Yield - 72mg, (67\%); mp: $131-134{ }^{\circ} \mathrm{C}$; $R f(20 \%$ EtOAc/Hexane) 0.3; IR (KBr, cm $\left.{ }^{-1}\right): 3065,1719,1681,{ }^{1} \mathbf{H} \mathbf{~ N M R}\left(400 \mathrm{MHz}, \mathrm{CDCl}_{3}\right): \delta 3.84(\mathrm{~s}$, $3 \mathrm{H}) 6.93(\mathrm{~d}, J=16.14 \mathrm{~Hz}, 1 \mathrm{H}) 7.26(\mathrm{~d}, J=8.07 \mathrm{~Hz}, 1 \mathrm{H}) 7.31(\mathrm{~m}, J=8.31 \mathrm{~Hz}, 2 \mathrm{H}) 7.34-7.41(\mathrm{~m}$, $1 \mathrm{H}) 7.57(\mathrm{~m}, J=8.31 \mathrm{~Hz}, 2 \mathrm{H}) 7.64(\mathrm{~d}, J=7.34 \mathrm{~Hz}, 1 \mathrm{H}) 7.98$ (s., $1 \mathrm{H}) 8.70$ (d, J=16.14 Hz, $1 \mathrm{H})$; ${ }^{13}$ C NMR (100 MHz, $\left.\mathrm{CDCl}_{3}\right): \delta 34.14,109.37,110.2,119.14,121.02,124.32,124.83,127.85$, 128.26, 128.68, 130.07, 132.73, 133.02, 136.42, 138.47, 140.9 (d, J=5.2Hz), 173.66 (d, J=33Hz, $\left.\mathbf{C O C F}_{3}\right)$; HRESI-MS $(\mathrm{m} / \mathrm{z})$ : Calculated for $\mathrm{C}_{19} \mathrm{H}_{13} \mathrm{ClF}_{3} \mathrm{NO}_{3}(\mathrm{M}+\mathrm{Na}): 386.0535$, found $(\mathrm{M}+$ $\mathrm{Na}): 386.0536$ 
13. (E)-1-(4-(4-bromostyryl)-1-methyl-1H-indol-3-yl)-2,2,2-trifluoroethan-1-one(4m):

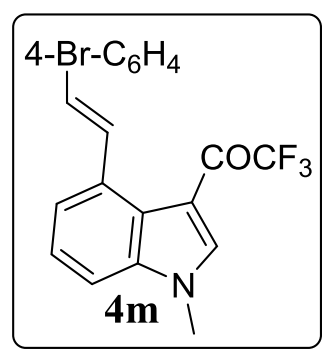

Prepared as shown in general procedure. Crude reaction mixture was purified on a flash column to obtain the product as Yellow solid; Yield - $60 \mathrm{mg},(50 \%) ; \boldsymbol{m p}$ : $144-147{ }^{\circ} \mathrm{C} ; R f(20$ $\%$ EtOAc/Hexane) 0.3; IR ( $\left.\mathrm{KBr}, \mathrm{cm}^{-1}\right): 3065,1719,1681,{ }^{1} \mathbf{H}$ NMR (800 MHz, $\left.\mathrm{CDCl}_{3}\right): \delta$ $3.87(\mathrm{~s}, 3 \mathrm{H}) 6.93(\mathrm{~d}, J=16.13 \mathrm{~Hz}, 1 \mathrm{H}) 7.28(\mathrm{~d}, J=7.99 \mathrm{~Hz}, 1 \mathrm{H}) 7.39$ (t, J=7.78 Hz, $1 \mathrm{H}) 7.47$ $(\mathrm{m}, J=8.42 \mathrm{~Hz}, 2 \mathrm{H}) 7.52(\mathrm{~m}, J=8.42 \mathrm{~Hz}, 2 \mathrm{H}) 7.66$ (d, $J=7.57 \mathrm{~Hz}, 1 \mathrm{H}) 7.99$ (s, $1 \mathrm{H}) 8.73$ (d, $J=16.13 \mathrm{~Hz}, 1 \mathrm{H}) ;{ }^{13} \mathbf{C ~ N M R}\left(200 \mathrm{MHz}, \mathrm{CDCl}_{3}\right): \delta 34.37,109.59,110.32,117.84(\mathrm{q}, J=293 \mathrm{~Hz}$, $\left.\mathrm{CF}_{3}\right), 121.09,121.2,124.46,125.01,128.33,128.47,130.32,131.78,133.13,136.97,138.62$, $141.15(\mathrm{~d}, J=4.9 \mathrm{~Hz}), 173.82\left(\mathrm{q}, J=33 \mathrm{~Hz}, \mathbf{C O C F}_{3}\right)$; HRESI-MS $(\mathrm{m} / \mathrm{z})$ : Calculated for $\mathrm{C}_{19} \mathrm{H}_{13} \mathrm{BrF}_{3} \mathrm{NO}_{3}(\mathrm{M}+\mathrm{Na}): 430.0030$, found $(\mathrm{M}+\mathrm{Na}): 430.003$

14. Methyl (E)-3-(3-acetyl-1-methyl-1H-indol-2-yl)acrylate (5a):

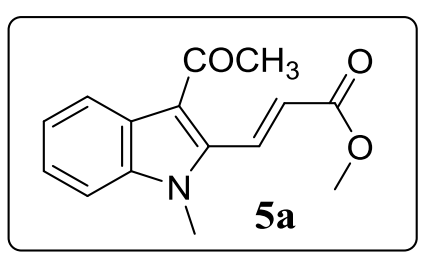

Prepared as shown in general procedure. Crude reaction mixture was purified on a flash column to obtain the product as Yellow solid; Yield - $61 \mathrm{mg},(80 \%) ; \boldsymbol{m p}: 109-111{ }^{\circ} \mathrm{C} ; R f(30 \%$ EtOAc/Hexane) 0.4; IR (KBr, cm $\left.{ }^{-1}\right): 1721,1641,{ }^{1} \mathbf{H} \mathbf{~ N M R}\left(400 \mathrm{MHz}, \mathrm{CDCl}_{3}\right): \delta 2.67(\mathrm{~s}, 3 \mathrm{H})$ $3.83(\mathrm{~s}, 3 \mathrm{H}) 3.86(\mathrm{~s}, 3 \mathrm{H}) 6.33(\mathrm{~d}, J=16.78 \mathrm{~Hz}, 1 \mathrm{H}) 7.28-7.42(\mathrm{~m}, 3 \mathrm{H}) 8.05(\mathrm{~d}, J=7.8 \mathrm{~Hz}, 1$ H) $8.22(\mathrm{~d}, J=16.48 \mathrm{~Hz}, 1 \mathrm{H}) ;{ }^{13} \mathbf{C} \mathbf{N M R}\left(100 \mathrm{MHz}, \mathrm{CDCl}_{3}\right)$ : $\delta 31.48,31.74,52.06,110.32$, $117.78,121.57,122.74,124.17,125.29,125.77,134.38,138.41$, 139.03, HRESI-MS $(\mathrm{m} / \mathrm{z})$ : Calculated for $\mathrm{C}_{15} \mathrm{H}_{15} \mathrm{NO}_{3}(\mathrm{M}+\mathrm{Na}): 280.0950$, found $(\mathrm{M}+\mathrm{Na}): 280.0844$. 
15. Methyl (E)-3-(3-acetyl-5-bromo-1-methyl-1H-indol-2-yl)acrylate (5b):

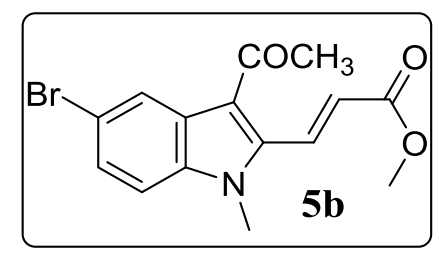

Prepared as shown in general procedure. Crude reaction mixture was purified on a flash column to obtain the product as Yellow solid; Yield - $68 \mathrm{mg}$, $(68 \%) ; \mathbf{m p}: 146-149{ }^{\circ} \mathrm{C} ; R f(30 \%$ EtOAc/Hexane) 0.4; IR (KBr, cm $\left.{ }^{-1}\right): 1697,1605,1451 ;{ }^{1} \mathbf{H} \mathbf{~ N M R}\left(400 \mathrm{MHz}, \mathrm{CDCl}_{3}\right): \delta 2.71$ $(\mathrm{s}, 3 \mathrm{H}) 3.81(\mathrm{~s}, 3 \mathrm{H}) 3.89(\mathrm{~s}, 3 \mathrm{H}) 6.52(\mathrm{~d}, J=16.48 \mathrm{~Hz}, 1 \mathrm{H}) 6.92(\mathrm{~m}, J=8.85 \mathrm{~Hz}, 2 \mathrm{H}) 7.13$ $(\mathrm{m}, J=8.85 \mathrm{~Hz}, 2 \mathrm{H}) 7.30-7.36(\mathrm{~m}, 1 \mathrm{H}) 7.36-7.45(\mathrm{~m}, 2 \mathrm{H}) 8.05(\mathrm{~d}, J=7.93 \mathrm{~Hz}, 1 \mathrm{H}) 8.40$ $(\mathrm{d}, J=16.48 \mathrm{~Hz}, 1 \mathrm{H}) ;{ }^{13} \mathbf{C}$ NMR $\left(100 \mathrm{MHz}, \mathrm{CDCl}_{3}\right): \delta 31.35,31.83,52.16,111.65,116.39$ ,116.95, 124.21, 126.28,127.09,127.30,133.83,136.89,139.86,165.98, 193.84.; HRESI-MS $(\mathrm{m} / \mathrm{z})$ : Calculated for $\mathrm{C}_{15} \mathrm{H}_{14} \mathrm{BrNO}_{3}(\mathrm{M}+\mathrm{Na}): 358.0055$, found $(\mathrm{M}+\mathrm{Na}): 358.0051$

16. Methyl (E)-3-(3-acetyl-5-methoxy-1-methyl-1H-indol-2-yl)acrylate (5c):

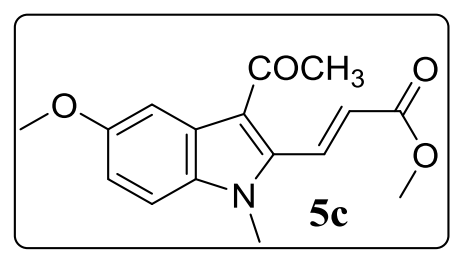

Prepared as shown in general procedure. Crude reaction mixture was purified on a flash column to obtain the product as Yellow solid; Yield - $67 \mathrm{mg},(78 \%) ; \mathbf{m p}: 146-148{ }^{\circ} \mathrm{C} ; R f(30 \%$ EtOAc/Hexane) 0.6; IR (KBr, cm $\left.{ }^{-1}\right): 1702,1618,{ }^{1} \mathbf{H} \mathbf{~ N M R}\left(400 \mathrm{MHz}, \mathrm{CDCl}_{3}\right): \delta 2.59$ (s, $\left.3 \mathrm{H}\right)$ $3.75(\mathrm{~s}, 3 \mathrm{H}) 3.85(\mathrm{~s}, 3 \mathrm{H}) 3.88(\mathrm{~s}, 3 \mathrm{H}) 6.26(\mathrm{~d}, J=16.48 \mathrm{~Hz}, 1 \mathrm{H}) 6.98(\mathrm{~d}, J=9.16 \mathrm{~Hz}, 1 \mathrm{H})$ $7.23(\mathrm{~d}, J=9.16 \mathrm{~Hz}, 1 \mathrm{H}) 7.52(\mathrm{~s}, 1 \mathrm{H}) 8.13(\mathrm{~d}, J=16.48 \mathrm{~Hz}, 1 \mathrm{H}) ;{ }^{13} \mathbf{C ~ N M R}\left(100 \mathrm{MHz}, \mathrm{CDCl}_{3}\right)$ : $\delta 31.25,31.75,51.97,55.67,103.21,110.93,114.46,117.32,125.05,126.55,133.54,134.20$ ,138.92,156.29, 166.14,194.03; HRESI-MS (m/z): Calculated for $\mathrm{C}_{16} \mathrm{H}_{17} \mathrm{NO}_{4}(\mathrm{M}+\mathrm{Na})$ : 310.1055 , found $(\mathrm{M}+\mathrm{Na}): 310.1059$. 


\section{Methyl(E)-3-acetyl-2-(3-methoxy-3-oxoprop-1-en-1-yl)-1-methyl-1H-indole-5-} carboxylate $(5 \mathrm{~d})$ :

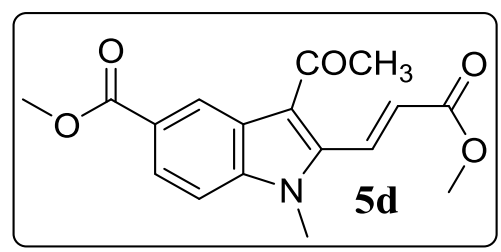

Prepared as shown in general procedure. Crude reaction mixture was purified on a flash column to obtain the product as Yellow solid; Yield - $66 \mathrm{mg}$, (70\%); mp: $146-149{ }^{\circ} \mathrm{C} ; R f(40 \%$ EtOAc/Hexane) 0.5; IR (KBr, cm $\left.{ }^{-1}\right): 1714,1697,{ }^{1} \mathbf{H} \mathbf{N M R}\left(400 \mathrm{MHz}, \mathrm{CDCl}_{3}\right): \delta 2.70(\mathrm{~s}, 3 \mathrm{H})$ $3.85(\mathrm{~s}, 3 \mathrm{H}) 3.87(\mathrm{~s}, 3 \mathrm{H}) 3.96(\mathrm{~s}, 3 \mathrm{H}) 6.36(\mathrm{~d}, J=16.48 \mathrm{~Hz}, 1 \mathrm{H}) 7.39(\mathrm{~d}, J=8.55 \mathrm{~Hz}, 1 \mathrm{H})$ $8.03(\mathrm{~d}, J=8.85 \mathrm{~Hz}, 1 \mathrm{H}) 8.16(\mathrm{~d}, J=16.48, \mathrm{~Hz}, 1 \mathrm{H}) 8.76(\mathrm{~s}, 1 \mathrm{H}) ;{ }^{13} \mathbf{C} \mathbf{N M R}\left(100 \mathrm{MHz}, \mathrm{CDCl}_{3}\right)$ : $\delta 31.53,31.91,52.09,52.13,76.68,77.31,110.04,118.41,124.09,124.56,125.19$, $125.24,126.11,133.73,140.33,140.51,166.00,167.40,194.08 ;$ HRESI-MS $(\mathrm{m} / \mathrm{z})$ : Calculated for $\mathrm{C}_{17} \mathrm{H}_{17} \mathrm{NO}_{5}(\mathrm{M}+\mathrm{Na}): 388.1004$, found $(\mathrm{M}+\mathrm{Na}): 388.1002$.

18. Methyl (E)-3-(1-methyl-3-propionyl-1H-indol-2-yl)acrylate (5e):

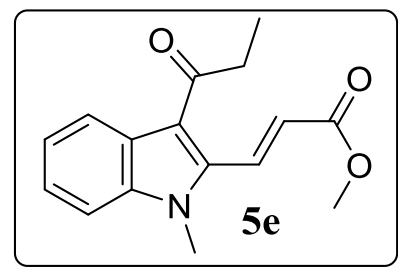

Prepared as shown in general procedure. Crude reaction mixture was purified on a flash column to obtain the product as Yellow solid; Yield - $68 \mathrm{mg},(84 \%)$; $\mathbf{m p}: 108-110{ }^{\circ} \mathrm{C} ; R f(30 \%$ EtOAc/Hexane) 0.4; IR ( $\left.\mathrm{KBr}, \mathrm{cm}^{-1}\right): 1711,1638,{ }^{1} \mathbf{H}$ NMR (400 MHz, $\left.\mathrm{CDCl}_{3}\right): \delta 1.25(\mathrm{t}$, $J=7.17 \mathrm{~Hz}, 3 \mathrm{H}) 2.95-3.05(\mathrm{~m}, 2 \mathrm{H}) 3.83(\mathrm{bs}, 3 \mathrm{H}) 3.85(\mathrm{~s}, 3 \mathrm{H}) 6.28$ (dd, J=16.63, $1.37 \mathrm{~Hz}$, $1 \mathrm{H}) 7.30$ (d, J=7.63 Hz, $1 \mathrm{H}) 7.32-7.40$ (m, $2 \mathrm{H}) 8.01$ (d, J=7.93 Hz, $1 \mathrm{H}) 8.23$ (dd, $J=16.48$, $1.53 \mathrm{~Hz}, 1 \mathrm{H}) ;{ }^{13} \mathbf{C}$ NMR $\left(100 \mathrm{MHz}, \mathrm{CDCl}_{3}\right): \delta 8.21,31.65,36.43,51.97,110.32,117.30$ ,121.58, $122.57,123.97,124.91,125.37,134.66,138.42,138.97,166.24,197.53$; HRESI-MS $(\mathrm{m} / \mathrm{z})$ : Calculated for $\mathrm{C}_{16} \mathrm{H}_{17} \mathrm{NO}_{3}(\mathrm{M}+\mathrm{Na}): 294.1106$, found $(\mathrm{M}+\mathrm{Na}): 294.1108$ 
19. Butyl (E)-3-(3-acetyl-1-methyl-1H-indol-2-yl)acrylate (5f):

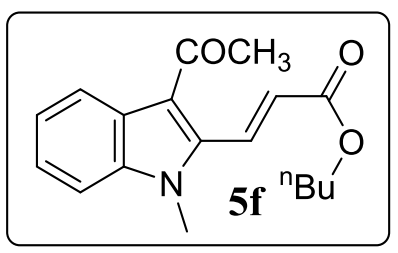

Prepared as shown in general procedure. Crude reaction mixture was purified on a flash column to obtain the product as Yellow solid; Yield - $73 \mathrm{mg},(83 \%)$; mp: $76-78{ }^{\circ} \mathrm{C}$; $R f(30 \%$ EtOAc/Hexane) 0.6; IR (KBr, cm $\left.{ }^{-1}\right): 1708,1632,{ }^{1} \mathbf{H} \mathbf{N M R}\left(400 \mathrm{MHz}, \mathrm{CDCl}_{3}\right): \delta 0.98(\mathrm{t}, J=7.32$ $\mathrm{Hz}, 3 \mathrm{H}) 1.42$ - $1.49(\mathrm{~m}, 2 \mathrm{H}) 1.68-1.77(\mathrm{~m}, 2 \mathrm{H}) 2.66(\mathrm{~s}, 3 \mathrm{H}) 3.82$ (s, $3 \mathrm{H}) 4.26$ (t, J=6.71 Hz, $2 \mathrm{H}) 6.32$ (d, J=16.78 Hz, $1 \mathrm{H}) 7.25-7.41(\mathrm{~m}, 3 \mathrm{H}) 8.05$ (d, J=7.93 Hz, $1 \mathrm{H}) 8.19$ (d, J=16.48 $\mathrm{Hz}, 1 \mathrm{H}) ;{ }^{13} \mathrm{C}$ NMR (100 MHz, $\left.\mathrm{CDCl}_{3}\right): \delta$ $13.68,19.11,30.63,31.43,31.69,64.88,110.25$ 194.27; HRESI-MS $(m / z)$ : Calculated for $\mathrm{C}_{18} \mathrm{H}_{21} \mathrm{NO}_{3}(\mathrm{M}+\mathrm{Na}): 322.1419$, found $(\mathrm{M}+\mathrm{Na}): 322.1417$.

\section{Phenyl (E)-3-(3-acetyl-1-methyl-1H-indol-2-yl)acrylate (5g):}

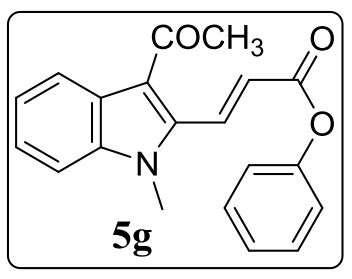

Prepared as shown in general procedure. Crude reaction mixture was purified on a flash column to obtain the product as Yellow solid; Yield - $66 \mathrm{mg},(70 \%) ; \mathbf{m p}: 135-137{ }^{\circ} \mathrm{C} ; R f(30 \%$ EtOAc/Hexane) 0.5; IR (KBr, cm $\left.{ }^{-1}\right): 3064,1709,1638,{ }^{1} \mathbf{H} \mathbf{N M R}\left(400 \mathrm{MHz}, \mathrm{CDCl}_{3}\right): \delta 2.70(\mathrm{~s}$, $3 \mathrm{H}) 3.88(\mathrm{~s}, 3 \mathrm{H}) 6.53(\mathrm{~d}, J=16.48 \mathrm{~Hz}, 1 \mathrm{H}) 7.18$ - $7.28(\mathrm{~m}, 4 \mathrm{H}) 7.30$ - 7.37 (m, $1 \mathrm{H}) 7.37$ - 7.45 $(\mathrm{m}, 3 \mathrm{H}) 8.04(\mathrm{~d}, J=8.24 \mathrm{~Hz}, 1 \mathrm{H}) 8.41(\mathrm{~d}, J=16.48 \mathrm{~Hz}, 1 \mathrm{H}) ;{ }^{13} \mathbf{C} \mathbf{N M R}\left(100 \mathrm{MHz}, \mathrm{CDCl}_{3}\right): \delta$ 129.41 , $135.83,138.56,138.60,150.64,164.33,194.41$; HRESI-MS $(\mathrm{m} / z)$ : Calculated for $\mathrm{C}_{20} \mathrm{H}_{17} \mathrm{NO}_{3}$ $(\mathrm{M}+\mathrm{Na}): 342.1106$, found $(\mathrm{M}+\mathrm{Na}): 342.1107$. 
21. 4-Methoxyphenyl (E)-3-(3-acetyl-1-methyl-1H-indol-2-yl)acrylate (5h):

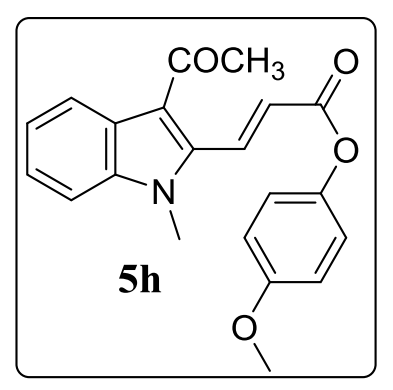

Prepared as shown in general procedure. Crude reaction mixture was purified on a flash column to obtain the product as Yellow solid; Yield - $76 \mathrm{mg},(74 \%) ; \mathbf{m p}: 137-139{ }^{\circ} \mathrm{C} ; R f(30 \%$ EtOAc/Hexane) 0.5; IR (KBr, cm $\left.{ }^{-1}\right): 1701,1677 ;{ }^{1} \mathbf{H}$ NMR (400 MHz, $\left.\mathrm{CDCl}_{3}\right): \delta 2.71(\mathrm{~s}, 3 \mathrm{H})$ $3.80(\mathrm{~s}, 3 \mathrm{H}) 3.89(\mathrm{~s}, 3 \mathrm{H}) 6.52(\mathrm{~d}, J=16.48 \mathrm{~Hz}, 1 \mathrm{H}) 6.92(\mathrm{~d}, J=8.85 \mathrm{~Hz}, 2 \mathrm{H}) 7.13(\mathrm{~d}, J=8.85$ $\mathrm{Hz}, 2 \mathrm{H})$ 7.31-7.37(m, 1H)7.39 - $7.43(\mathrm{~m}, 2 \mathrm{H}) 8.05$ (d, J=8.0 Hz, $1 \mathrm{H}) 8.40$ (d, J=16.48 Hz, 1 $\mathrm{H}) ;{ }^{13} \mathrm{C}$ NMR $\left(100 \mathrm{MHz}, \mathrm{CDCl}_{3}\right): \delta$ $31.48,31.79,55.4,110.40,114.37,118.04,121.48,122.19$ ,122.73,124.28, 125.63, 135.60,138.52, 138.56, 144.07, 157.24, 164.64, HRESI-MS $(\mathrm{m} / \mathrm{z})$ : Calculated for $\mathrm{C}_{21} \mathrm{H}_{19} \mathrm{NO}_{4}(\mathrm{M}+\mathrm{Na}): 372.1212$, found $(\mathrm{M}+\mathrm{Na}): 372.1212$

\section{Naphthalen-1-yl (E)-3-(3-acetyl-1-methyl-1H-indol-2-yl)acrylate (5i):}

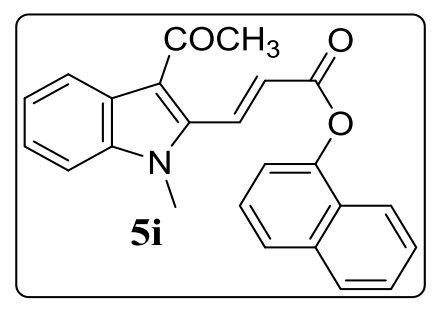

Prepared as shown in general procedure. Crude reaction mixture was purified on a flash column to obtain the product as Yellow solid; Yield - $70 \mathrm{mg},(65 \%) ; \mathbf{m p}: 142-145{ }^{\circ} \mathrm{C} ; R f(30 \%$ EtOAc/Hexane) 0.5; IR (KBr, cm $\left.{ }^{-1}\right): 1703,1634 ;{ }^{1} \mathbf{H} \mathbf{N M R}\left(400 \mathrm{MHz}, \mathrm{CDCl}_{3}\right): \delta 2.73(\mathrm{~s}, 3 \mathrm{H})$ $3.90(\mathrm{~s}, 3 \mathrm{H}) 6.59$ (d, J=16.48 Hz, $1 \mathrm{H}) 7.32-7.42(\mathrm{~m}, 4 \mathrm{H}) 7.48$ (t, J=6.56 Hz, 2 H) 7.69 (s, 1 H) $7.80-7.90(\mathrm{~m}, 3 \mathrm{H}) 8.05(\mathrm{~d}, J=8.24 \mathrm{~Hz}, 1 \mathrm{H}) 8.47$ (d, J=16.48 Hz, $1 \mathrm{H})$; ${ }^{13}$ C NMR (100 $\left.\mathrm{MHz}, \mathrm{CDCl}_{3}\right)$ : $\delta 31.63,31.88,110.54,118.26,118.56,121.07,121.61,122.86,124.21,124.44$, $125.74,125.78,126.61,127.71,127.79,129.44,131.51,133.76,136.01,138.55,138.66,148.38$, 164.58, 194.45.; HRESI-MS ( $\mathrm{m} / \mathrm{z})$ : Calculated for $\mathrm{C}_{24} \mathrm{H}_{19} \mathrm{NO}_{3}(\mathrm{M}+\mathrm{Na}): 392.1263$, found $(\mathrm{M}+$ $\mathrm{Na}): 392.1267$. 
23. Dimethyl 3,3'-(1-methyl-3-pivaloyl-1H-indole-2,4-diyl)(2E,2'E)-diacrylate (6a):

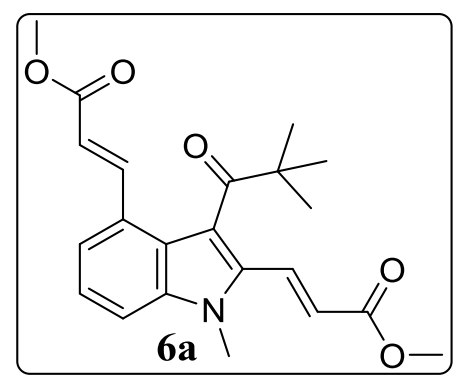

Prepared as shown in general procedure. Crude reaction mixture was purified on a flash column to obtain the product as Yellow solid; Yield - $57 \mathrm{mg},(50 \%) ; R f$ (30\% EtOAc/Hexane) 0.4; IR $\left(\mathrm{KBr}, \mathrm{cm}^{-1}\right):, 1721,1643,{ }^{1} \mathbf{H}$ NMR $\left(400 \mathrm{MHz}, \mathrm{CDCl}_{3}\right): \delta 1.20(\mathrm{~s}, 9 \mathrm{H}) 3.81(\mathrm{~s}, 6 \mathrm{H}) 3.84(\mathrm{~s}, 3 \mathrm{H})$ $6.32(\mathrm{~d}, J=16.14 \mathrm{~Hz}, 1 \mathrm{H}) 6.47(\mathrm{~d}, J=15.89 \mathrm{~Hz}, 1 \mathrm{H}) 7.28-7.40(\mathrm{~m}, 2 \mathrm{H}) 7.45(\mathrm{~d}, J=7.09 \mathrm{~Hz}, 1$ H) $7.71(\mathrm{~d}, J=16.14 \mathrm{~Hz}, 1 \mathrm{H}) 7.83(\mathrm{~d}, J=15.65 \mathrm{~Hz}, 1 \mathrm{H}) ;{ }^{13} \mathbf{C} \mathbf{~ N M R}\left(100 \mathrm{MHz}, \mathrm{CDCl}_{3}\right)$ : $\delta 27.51$, $31.21,46.94,51.65,51.99,111.48,118.57,119.07,119.44,122.66,124.21,124.67,127.78$, $131.75,132.30,138.46,142.20,166.58,166.93,214.18$; HRESI-MS $(\mathrm{m} / \mathrm{z})$ : Calculated for $\mathrm{C}_{22} \mathrm{H}_{25} \mathrm{NO}_{5}(\mathrm{M}+\mathrm{Na}): 406.1630$, found $(\mathrm{M}+\mathrm{Na}): 406.1628$

\section{Methyl (E)-3-(1-methyl-3-(methylcarbamoyl)-1H-indol-2-yl)acrylate (9a):}

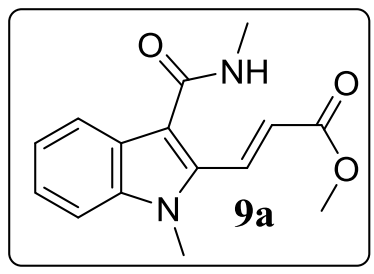

Prepared as shown in general procedure. Crude reaction mixture was purified on a flash column to obtain the product as Yellow solid; Yield $-60 \mathrm{mg}$, (74\%); $\mathbf{m p}$ : $192-194{ }^{\circ} \mathrm{C} ; R f(50 \%$ EtOAc/Hexane) $0.4 ; \mathbf{I R}\left(\mathrm{KBr}, \mathrm{cm}^{-1}\right): 3064,1638 ;{ }^{1} \mathbf{H} \mathbf{~ N M R}\left(400 \mathrm{MHz}, \mathrm{CDCl}_{3}\right): \delta 3.05$ (d, J=4.88 $\mathrm{Hz}, 3 \mathrm{H}) 3.77$ (s, $3 \mathrm{H}$ ) 3.82 (s, $3 \mathrm{H}$ ) 6.03 (br. s., $1 \mathrm{H}$ ) 6.50 (d, $J=16.48 \mathrm{~Hz}, 1 \mathrm{H}$ ) 7.21 (ddd, $J=8.24$, $4.73,3.20 \mathrm{~Hz}, 1 \mathrm{H}) 7.32$ (d, $J=3.66 \mathrm{~Hz}, 2 \mathrm{H}) 7.78(\mathrm{~d}, J=7.93 \mathrm{~Hz}, 1 \mathrm{H}) 8.11(\mathrm{~d}, J=16.48 \mathrm{~Hz}, 1 \mathrm{H})$; ${ }^{13}$ C NMR (100 MHz, $\left.\mathrm{CDCl}_{3}\right): \delta$ : $26.61,31.45,51.90,110.09,113.77,120.15,121.71,73$ 124.28, 125.16, 132.44, 135.05, 138.20,165.68.; HRESI-MS (m/z): Calculated for $\mathrm{C}_{15} \mathrm{H}_{16} \mathrm{~N}_{2} \mathrm{O}_{3}(\mathrm{M}+\mathrm{Na}): 295.1059$, found $(\mathrm{M}+\mathrm{Na}): 295.1057$. 
25. Butyl (E)-3-(1-methyl-3-(methylcarbamoyl)-1H-indol-2-yl)acrylate (9b):

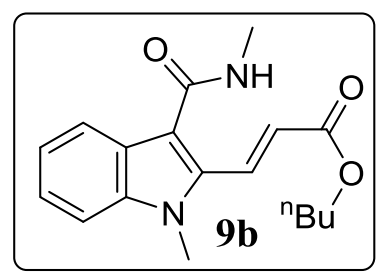

Prepared as shown in general procedure. Crude reaction mixture was purified on a flash column to obtain the product as Yellow solid; Yield - $82 \mathrm{mg},(88 \%) ; \boldsymbol{m p}: 155-158{ }^{\circ} \mathrm{C} ; R f(50 \%$ EtOAc/Hexane) 0.4; IR (KBr, cm $\left.{ }^{-1}\right): 3064,1671,1457,{ }^{1} \mathbf{H} \mathbf{N M R}\left(400 \mathrm{MHz}, \mathrm{CDCl}_{3}\right): \delta 0.97(\mathrm{t}$, $J=7.32 \mathrm{~Hz}, 3 \mathrm{H}) 1.40-1.48(\mathrm{~m}, 2 \mathrm{H}) 1.70(\mathrm{t}, J=7.02 \mathrm{~Hz}, 2 \mathrm{H}) 3.02$ (d, J=4.58 Hz, $3 \mathrm{H}) 3.70$ (s, $3 \mathrm{H}) 4.21$ (t, J=6.71 Hz, $2 \mathrm{H}) 6.11$ (br. s., $1 \mathrm{H}) 6.45$ (d, J=16.48 Hz, $1 \mathrm{H}) 7.13-7.22$ (m, $1 \mathrm{H})$ 7.25 - $7.33(\mathrm{~m}, 2 \mathrm{H}) 7.76(\mathrm{~d}, J=7.93 \mathrm{~Hz}, 1 \mathrm{H}) 8.02$ (d, J=16.17 Hz, $1 \mathrm{H}) ;{ }^{13} \mathrm{C} \mathrm{NMR}(100 \mathrm{MHz}$,

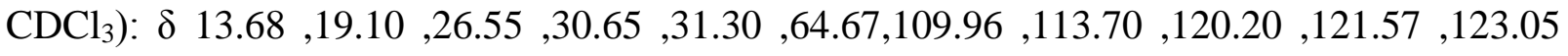
124.15, 125.19, 132.04, 134.87, 138.06, 165.66. HRESI-MS (m/z): Calculated for $\mathrm{C}_{18} \mathrm{H}_{22} \mathrm{~N}_{2} \mathrm{O}_{3}(\mathrm{M}+\mathrm{Na}): 337.1528$, found $(\mathrm{M}+\mathrm{Na}): 337.1526$

26. Methyl(E)-4-(3-methoxy-3-oxoprop-1-en-1-yl)-1-methyl-1H-indole-3-carboxylate(10a):

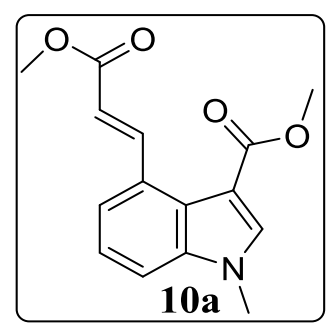

Prepared as shown in general procedure. Crude reaction mixture was purified on a flash column to obtain the product as Yellow solid; Yield - $32 \mathrm{mg},(39 \%) ; \boldsymbol{m p}: 103-105{ }^{\circ} \mathrm{C}$; $R f(30 \%$ EtOAc/Hexane) 0.4; IR (KBr, cm $\left.{ }^{-1}\right): 1766,1638,1492 ;{ }^{1} \mathbf{H} \mathbf{N M R}\left(400 \mathrm{MHz} \mathrm{CDCl}_{3}\right): \delta 3.82(\mathrm{~s}$, $3 \mathrm{H}) 3.84(\mathrm{~s}, 3 \mathrm{H}) 3.90(\mathrm{~s}, 3 \mathrm{H}) 6.40(\mathrm{~d}, J=15.87 \mathrm{~Hz}, 1 \mathrm{H}) 7.30(\mathrm{t}, J=7.78 \mathrm{~Hz}, 1 \mathrm{H}) 7.36$ (d, J=7.93 $\mathrm{Hz}, 1 \mathrm{H}) 7.53$ (d, J=7.32 Hz, $1 \mathrm{H}) 7.90$ (s, $1 \mathrm{H}) 9.31$ (d, J=15.87 Hz, $1 \mathrm{H}) ;{ }^{13} \mathbf{C ~ N M R}(100 \mathrm{MHz}$, $\left.\mathrm{CDCl}_{3}\right): \delta 33.56,51.37,51.60,107.62,111.43,117.71,120.80,122.91,124.86,129.11,137.92$ ,138.26, 146.27, 164.84,167.80.; HRESI-MS (m/z): Calculated for $\mathrm{C}_{15} \mathrm{H}_{15} \mathrm{NO}_{4}(\mathrm{M}+\mathrm{Na})$ : 296.0899, found $(\mathrm{M}+\mathrm{Na}): 296.0896$. 
27. Methyl(E)-4-(3-butoxy-3-oxoprop-1-en-1-yl)-1-methyl-1H-indole-3-carboxylate(10b):

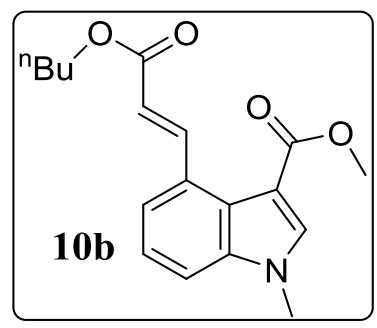

Prepared as shown in general procedure. Crude reaction mixture was purified on a flash column to obtain the product as Yellow solid; Yield - $44 \mathrm{mg},(47 \%) ; \mathbf{m p}: 81-83{ }^{\circ} \mathrm{C} ; R f(30 \%$ EtOAc/Hexane) 0.4; IR (KBr, cm $\left.{ }^{-1}\right): 1671,1623,1458 ;{ }^{1} \mathbf{H ~ N M R}\left(400 \mathrm{MHz} \mathrm{CDCl}_{3}\right): \delta 0.98(\mathrm{t}$, $J=7.32 \mathrm{~Hz}, 3 \mathrm{H}) 1.45-1.54(\mathrm{~m}, 2 \mathrm{H}) 1.68-1.76(\mathrm{~m}, 2 \mathrm{H}) 3.82(\mathrm{~s}, 3 \mathrm{H}) 3.89(\mathrm{~s}, 3 \mathrm{H}) 4.24(\mathrm{t}$, $J=6.56 \mathrm{~Hz}, 2 \mathrm{H}) 6.39$ (d, J=15.87 Hz, $1 \mathrm{H}) 7.30(\mathrm{~d}, J=7.63 \mathrm{~Hz}, 1 \mathrm{H}) 7.32$ - 7.39 (m, $1 \mathrm{H}) 7.54$ (d, $J=7.32 \mathrm{~Hz}, 1 \mathrm{H}) 7.89$ (s, $1 \mathrm{H}) 9.30$ (d, J=15.87 Hz, $1 \mathrm{H}) ;{ }^{13} \mathbf{C ~ N M R}\left(100 \mathrm{MHz}, \mathrm{CDCl}_{3}\right): \delta 13.78$ 129.19, 124.86, 122.89, 120.74, 118.14, 111.33, 19.67.68, 30.86, 33.55, 51.31, ,137.80, 138.26, 145.96, 164.80,167.49.; HRESI-MS $(\mathrm{m} / \mathrm{z})$ : Calculated for $\mathrm{C}_{18} \mathrm{H}_{21} \mathrm{NO}_{4}(\mathrm{M}+$ $\mathrm{Na}): 338.1368$, found $(\mathrm{M}+\mathrm{Na}): 338.1370$

References:

1. a) Kong, A; Han, X.; Lu, X. Org. Lett. 2006 , 8, 1339.

b) Park, C.M.; Kim, S.Y; Park, W.K; Park, N.S; Seong, C.M. Bioorg. Med. Chem. Lett. 2008, 18, 3844.

2. a) Guchhait, S. K.; Kashyap, M.; Kamble,H. J. Org. Chem. 2011, 76, 4753.

b) Okauchi, T.; Itonaga, M.; Minami, T.; Owa, T.; Kitoh, K.; Yoshino, H. Org. Lett. 2000, 2 , 1485. 


\section{Computational Methods}

All stationary points, such as, reactants, intermediates, and transition states were fully optimized using Gaussian09 suite of quantum chemical programs. ${ }^{1}$

1) Calculated $\mathrm{pKa}$ Values ${ }^{2}$

Theory Level- M06

Basis set - 6-311+g (2d,p)

Solvent - dichloroethane (PCM)

Temperature - $298.15 \mathrm{~K}$

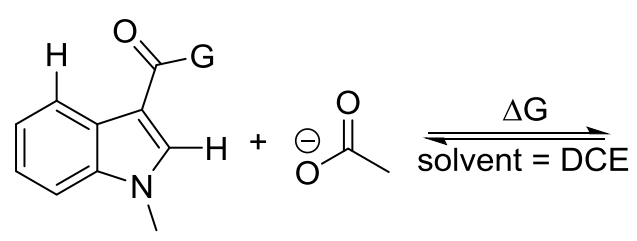<smiles></smiles><smiles>CC(=O)O</smiles><smiles>C#CC(=C=C)C(=O)O</smiles><smiles>CC(=O)c1c(C(C)(C)C)n(C)c2cccc([AsH3])c12</smiles>

The $\Delta \mathrm{G}$ values were converted to $\mathrm{pKa}$ values according to the following equation.

$$
\mathrm{p} K_{a}(\text { Indole }-H)=\mathrm{p} K_{a}(A c O H)+\frac{\Delta G_{\text {exchange }}}{2.303 \times R T}
$$

$\mathrm{pKa}(\mathrm{AcOH})=15.5^{3}$

${ }^{1}$ Gaussian 09, Revision D.01, Frisch, M. J.; Trucks, G. W.; Schlegel, H. B.; Scuseria, G. E.; Robb, M. A.; Cheeseman, J. R.; Scalmani, G.; Barone, V.; Mennucci, B.; Petersson, G. A.; Nakatsuji, H.; Caricato, M.; Li, X.; Hratchian, H. P.; Izmaylov, A. F.; Bloino, J.; Zheng, G.; Sonnenberg, J. L.; Hada, M.; Ehara, M.; Toyota, K.; Fukuda, R.; Hasegawa, J.; Ishida, M.; Nakajima, T.; Honda, Y.; Kitao, O.; Nakai, H.; Vreven, T.; Montgomery, J. A., Jr.; Peralta, J. E.; Ogliaro, F.; Bearpark, M.; Heyd, J. J.; Brothers, E.; Kudin, K. N.; Staroverov, V. N.; Kobayashi, R.; Normand, J.; Raghavachari, K.; Rendell, A.; Burant, J. C.; Iyengar, S. S.; Tomasi, J.; Cossi, M.; Rega, N.; Millam, J. M.; Klene, M.; Knox, J. E.; Cross, J. B.; Bakken, V.; Adamo, C.; Jaramillo, J.; Gomperts, R.; Stratmann, R. E.; Yazyev, O.; Austin, A. J.; Cammi, R.; Pomelli, C.; Ochterski, J. W.; Martin, R. L.; Morokuma, K.; Zakrzewski, V. G.; Voth, G. A.; Salvador, P.; Dannenberg, J. J.; Dapprich, S.; Daniels, A. D.; Farkas, Ö.; Foresman, J. B.; Ortiz, J. V.; Cioslowski, J.; Fox, D. J. Gaussian, Inc., Wallingford CT, 2009.

${ }^{2}$ Shen, K.; Fu, Y.; Li, J.-N.; Liu, L.; Guo, Q.-X. Tetrahedron 2007, 63, 1568.

${ }^{3}$ Raamat, E.; Kaupmees, K.; Ovsjannikov, G.; Trummal, A.; Kutt, A.; Saame, J.; Koppel, I.; Kaljurand, I.; Lipping, L.; Rodima, T.; Pihl, V.; Koppel, I. A.; Leito, I. J. Phys. Org. Chem. 2013, 26, 162 - 170. 


\section{2) Partial Charges on carbon atoms of 1a and $2 \mathbf{a}$.}

Were obtained by using the CHELPG program from the optimized structures of the two limiting orientations, using the keyword $\mathrm{POP}=\mathrm{CHELPG}$ at the M06 Level, 6-31G* Basis set at $373 \mathrm{~K}$ in dichloroethane.

\section{Diagram of Partial Charges on all atoms:}
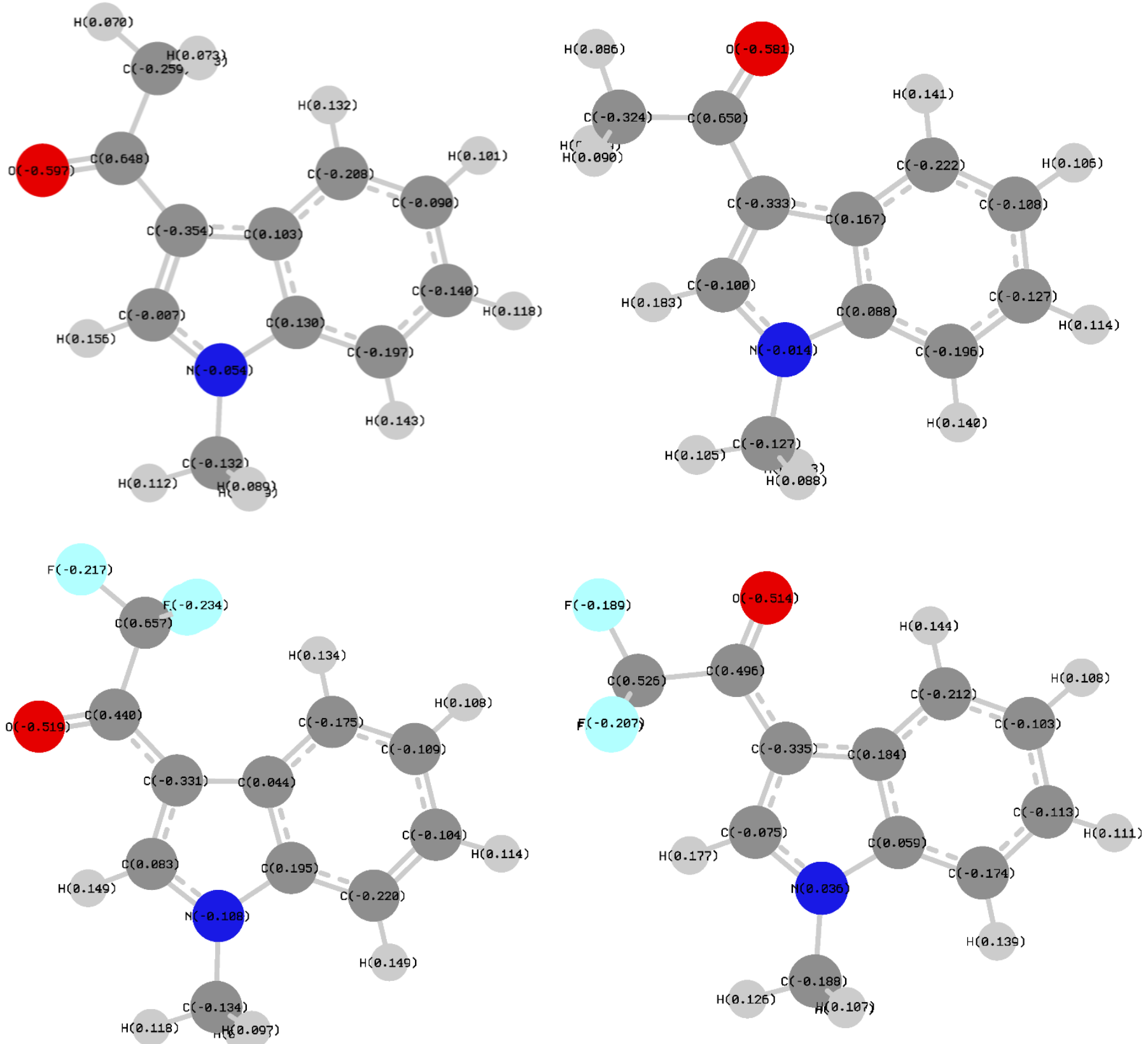
Table of Energies

\begin{tabular}{|l|l|c|c|c|l|}
\hline \multicolumn{1}{|l|}{ Structure } & $\begin{array}{c}\mathbf{E}_{\text {elec }} \\
\text { (Hartree) }\end{array}$ & $\begin{array}{c}\mathbf{E}_{\text {elec }}+\mathbf{Z P E} \\
\text { (Hartree) }\end{array}$ & $\begin{array}{c}\mathbf{H} \\
\text { (Hartree) }\end{array}$ & $\begin{array}{c}\text { G } \\
\text { (Hartree) }\end{array}$ & $\begin{array}{c}\text { No. of } \\
\text { imaginary } \\
\text { frequencies }\end{array}$ \\
\hline Calculation of $p K_{a}$ values \\
\hline 1a & -853.276840060 & -853.105089 & -853.090971 & -853.145457 & 0 \\
\hline 1a C2-anion & -852.748994625 & -852.591034 & -852.577042 & -852.631474 & 0 \\
\hline 1a C4-anion & -852.717304377 & -852.559815 & -852.545645 & -852.600474 & 0 \\
\hline 2a & -555.538895511 & -555.345352 & -555.332967 & -555.382783 & 0 \\
\hline 2a C2-anion & -555.002143591 & -554.821769 & -554.809785 & -554.858360 & 0 \\
\hline 2a C4-anion & -554.971856130 & -554.792410 & -554.779964 & -554.829925 & 0 \\
\hline Acetic acid & -229.042588978 & -228.981188 & -228.975674 & -229.008396 & 0 \\
\hline Acetate & -228.567476414 & -228.519181 & -228.513889 & -228.546326 & 0 \\
\hline
\end{tabular}

\section{Coordinates of structures}

\begin{tabular}{|c|c|c|c|}
\hline \multicolumn{4}{|l|}{$1 \mathbf{a}$} \\
\hline \multicolumn{4}{|r|}{0.000038000} \\
\hline 8 & -2.168271000 & 2.059955000 & 0.000083000 \\
\hline 6 & 0.483452000 & -1.782053000 & -0.000021000 \\
\hline 6 & 0.658252000 & -0.396052000 & 0.000000000 \\
\hline 6 & 1.977761000 & 0.098716000 & -0.000001000 \\
\hline 6 & 3.100580000 & -0.714739000 & -0.000025000 \\
\hline 6 & 2.892219000 & -2.079212000 & -0.000047000 \\
\hline 6 & 1.595907000 & -2.601021000 & -0.000044000 \\
\hline 6 & -0.200402000 & 0.771899000 & 0.000026000 \\
\hline 6 & 0.644960000 & 1.870673000 & 0.000043000 \\
\hline 7 & 1.926852000 & 1.483928000 & 0.000026000 \\
\hline 1 & -0.503319000 & -2.226354000 & -0.000019000 \\
\hline 1 & 4.101106000 & -0.294072000 & -0.000027000 \\
\hline 1 & 3.742512000 & -2.753096000 & -0.000067000 \\
\hline 1 & 1.458577000 & -3.677636000 & -0.000061000 \\
\hline 6 & 3.085376000 & 2.348569000 & 0.000020000 \\
\hline 1 & 2.754507000 & 3.386192000 & 0.000068000 \\
\hline 1 & 3.693217000 & 2.166085000 & 0.889495000 \\
\hline 1 & 3.693163000 & 2.166148000 & -0.889506000 \\
\hline 6 & -2.535581000 & -0.270286000 & -0.000011000 \\
\hline 9 & -2.312752000 & -1.026066000 & 1.078821000 \\
\hline 9 & -3.811975000 & 0.064666000 & -0.000022000 \\
\hline 9 & -2.312718000 & -1.026012000 & -1.078872000 \\
\hline 1 & 0.374940000 & 2.917974000 & 0.000064000 \\
\hline
\end{tabular}




\begin{tabular}{|c|c|c|c|}
\hline \multicolumn{4}{|c|}{ 1a C2-anion } \\
\hline 6 & 1.598912000 & 1.020526000 & -0.008958000 \\
\hline 8 & 2.230497000 & 2.071908000 & -0.021081000 \\
\hline 6 & -0.459661000 & -1.732209000 & -0.009309000 \\
\hline 6 & -0.638332000 & -0.346704000 & -0.004475000 \\
\hline 6 & -1.966141000 & 0.127048000 & 0.000742000 \\
\hline 6 & -3.074945000 & -0.702960000 & 0.003123000 \\
\hline 6 & -2.860947000 & -2.073391000 & -0.000831000 \\
\hline 6 & -1.562147000 & -2.574904000 & -0.007358000 \\
\hline 6 & 0.187310000 & 0.857516000 & -0.006483000 \\
\hline 6 & -0.653073000 & 2.014130000 & -0.003073000 \\
\hline 7 & -1.916905000 & 1.517011000 & 0.001941000 \\
\hline 1 & 0.527245000 & -2.174414000 & -0.014864000 \\
\hline 1 & -4.081553000 & -0.294442000 & 0.007598000 \\
\hline 1 & -3.706379000 & -2.754806000 & 0.000716000 \\
\hline 1 & -1.404367000 & -3.649859000 & -0.011147000 \\
\hline 6 & -3.116645000 & 2.316923000 & 0.009249000 \\
\hline 1 & -2.825497000 & 3.366956000 & 0.009509000 \\
\hline 1 & -3.731293000 & 2.114044000 & -0.875295000 \\
\hline 1 & -3.722241000 & 2.111131000 & 0.899355000 \\
\hline 6 & 2.485503000 & -0.251423000 & 0.005806000 \\
\hline 9 & 2.282631000 & -1.019471000 & -1.074803000 \\
\hline 9 & 3.777454000 & 0.050565000 & 0.018875000 \\
\hline 9 & 2.259853000 & -1.013346000 & 1.085771000 \\
\hline \multicolumn{4}{|c|}{ 1a C4-anion } \\
\hline 6 & 1.659646000 & -2.016689000 & -0.000001000 \\
\hline 6 & 1.147178000 & -0.698549000 & -0.000001000 \\
\hline 6 & 1.971476000 & 0.448936000 & 0.000000000 \\
\hline 6 & 3.357071000 & 0.387807000 & 0.000001000 \\
\hline 6 & 3.894356000 & -0.888067000 & 0.000001000 \\
\hline 6 & 3.066464000 & -2.021765000 & 0.000000000 \\
\hline 6 & -0.218345000 & -0.171147000 & -0.000002000 \\
\hline 6 & -0.118804000 & 1.213716000 & -0.000001000 \\
\hline 7 & 1.170945000 & 1.586325000 & 0.000000000 \\
\hline 1 & 3.976458000 & 1.280345000 & 0.000001000 \\
\hline 1 & 4.977390000 & -1.007607000 & 0.000001000 \\
\hline 1 & 3.592281000 & -2.982473000 & 0.000000000 \\
\hline 1 & -0.892414000 & 1.969898000 & -0.000001000 \\
\hline 6 & -1.455294000 & -0.884139000 & -0.000002000 \\
\hline 8 & -1.619597000 & -2.087499000 & 0.000001000 \\
\hline 6 & 1.663964000 & 2.940814000 & 0.000000000 \\
\hline 1 & 2.275295000 & 3.125388000 & -0.888109000 \\
\hline 1 & 2.275293000 & 3.125388000 & 0.888111000 \\
\hline 1 & 0.820694000 & 3.631826000 & 0.000000000 \\
\hline
\end{tabular}




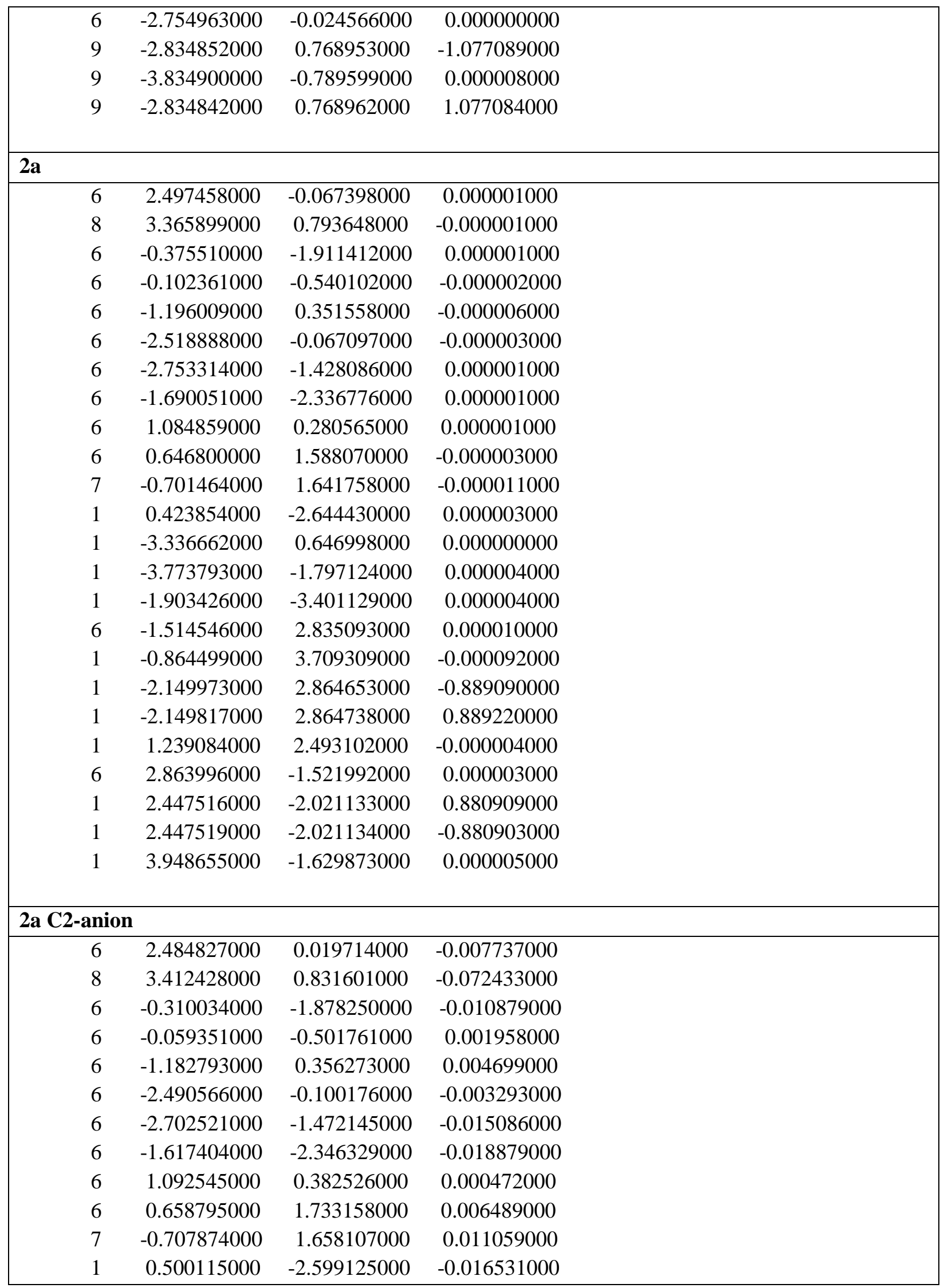




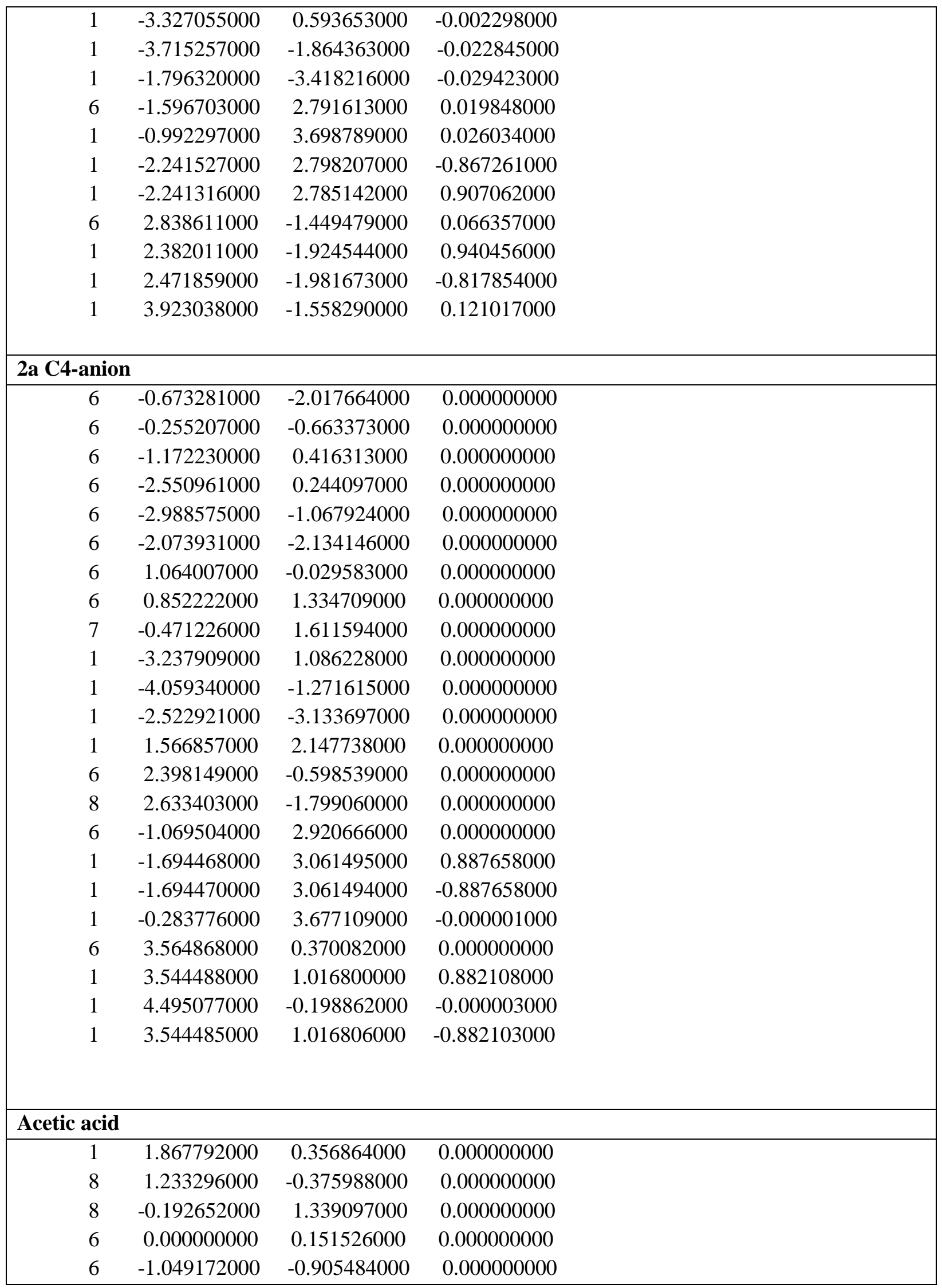




\begin{tabular}{|rrrr|}
\hline 1 & -0.929859000 & -1.542797000 & 0.879293000 \\
1 & -2.038198000 & -0.452401000 & 0.000000000 \\
1 & -0.929859000 & -1.542797000 & -0.879293000 \\
\hline & & & \\
\hline Acetate & & & \\
\hline 8 & -0.753268000 & 1.118576000 & 0.001843000 \\
8 & -0.753292000 & -1.118565000 & 0.001843000 \\
6 & -0.190520000 & 0.000000000 & -0.010476000 \\
6 & 1.338238000 & -0.000011000 & -0.004935000 \\
1 & 1.683521000 & 0.000026000 & 1.034854000 \\
1 & 1.741323000 & -0.894417000 & -0.485903000 \\
1 & 1.741329000 & 0.894359000 & -0.485965000 \\
\hline
\end{tabular}


Lanke, Bettadapur, and Prabhu/Org Chem/IISc

Vె舟

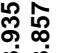

:

LVR-06144A

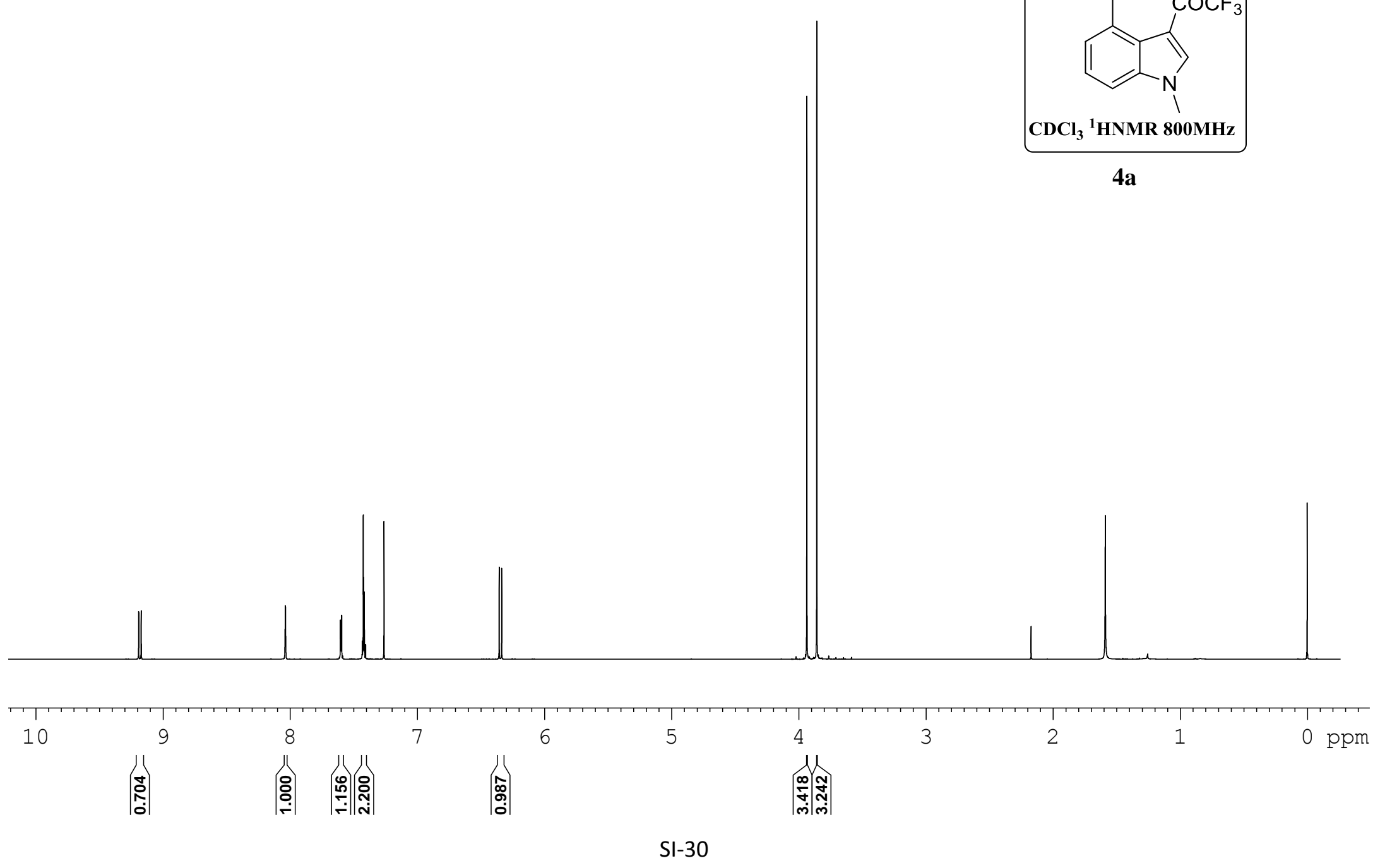




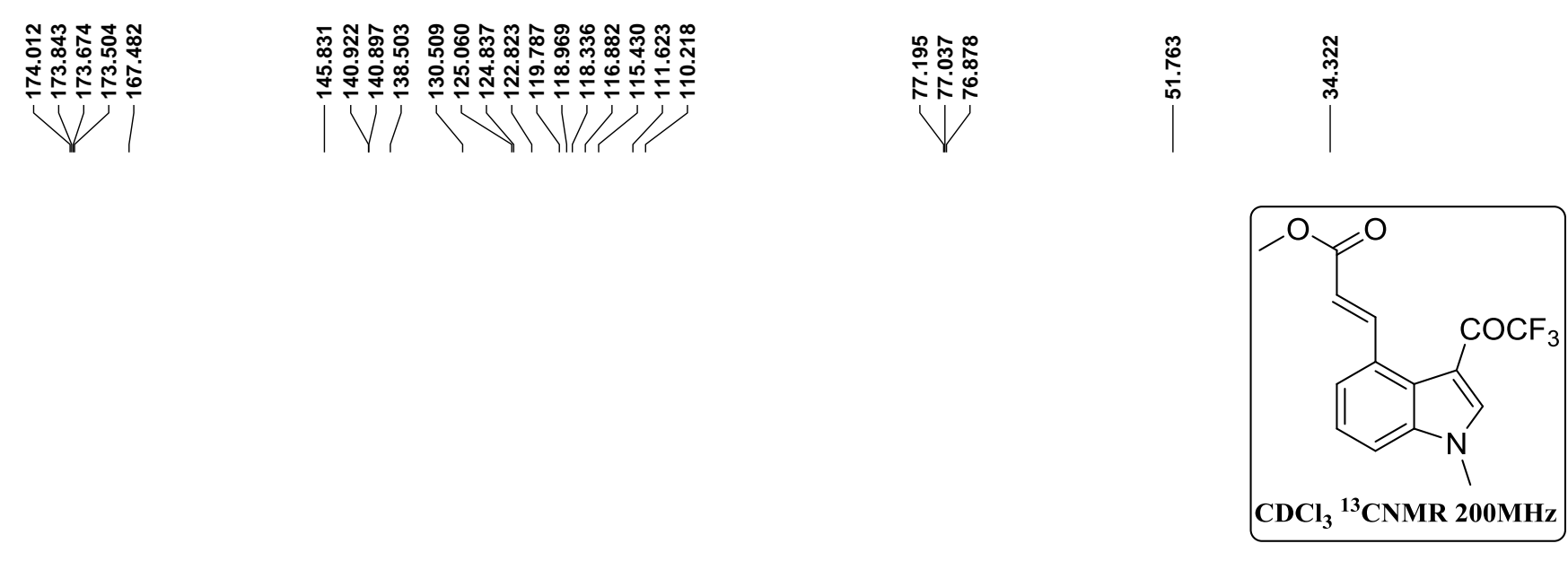

$4 a$

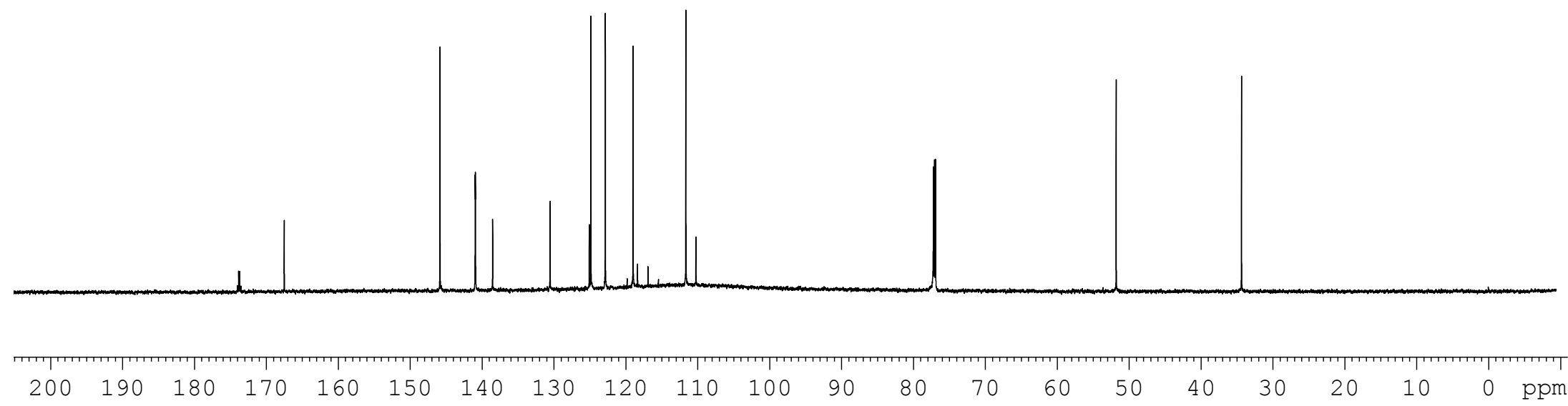


Lanke, Bettadapur, and Prabhu/Org Chem/IISc

Vi

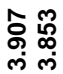

:

LVR-06165A

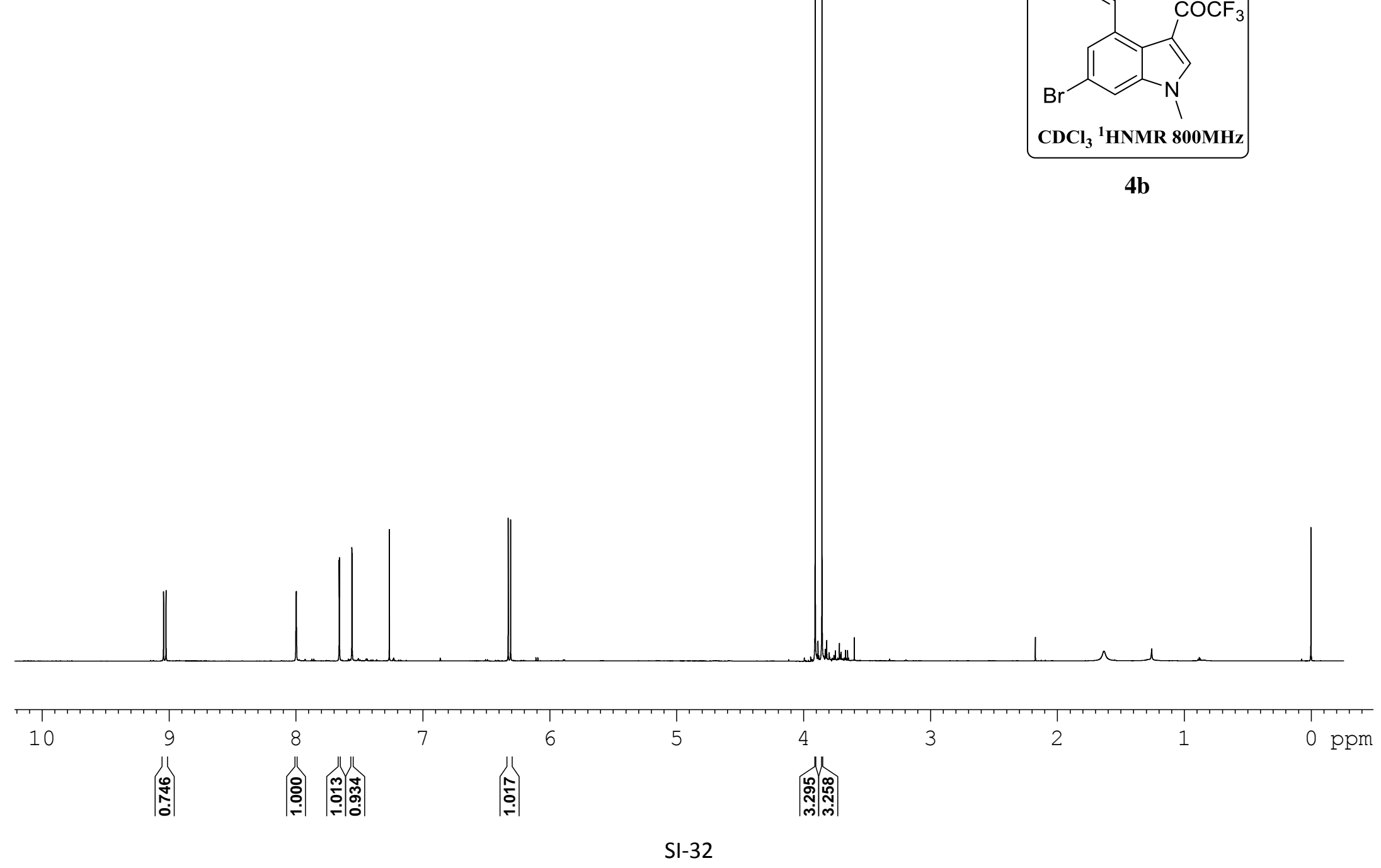




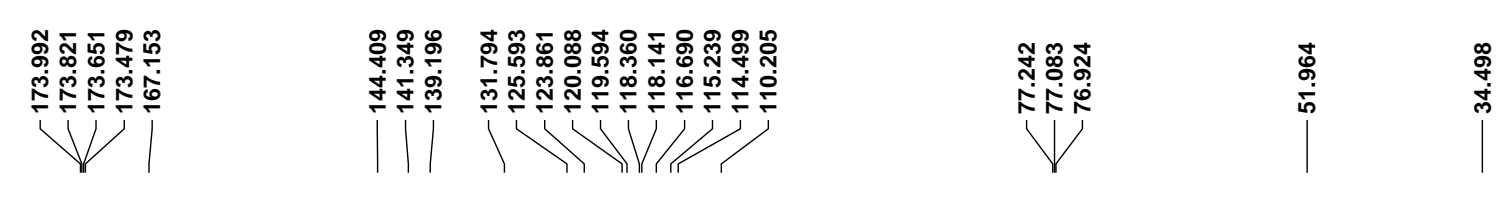

LVR-06165A

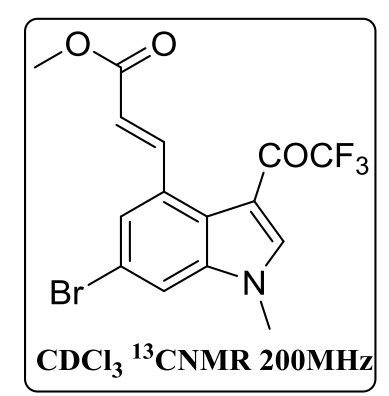

4b

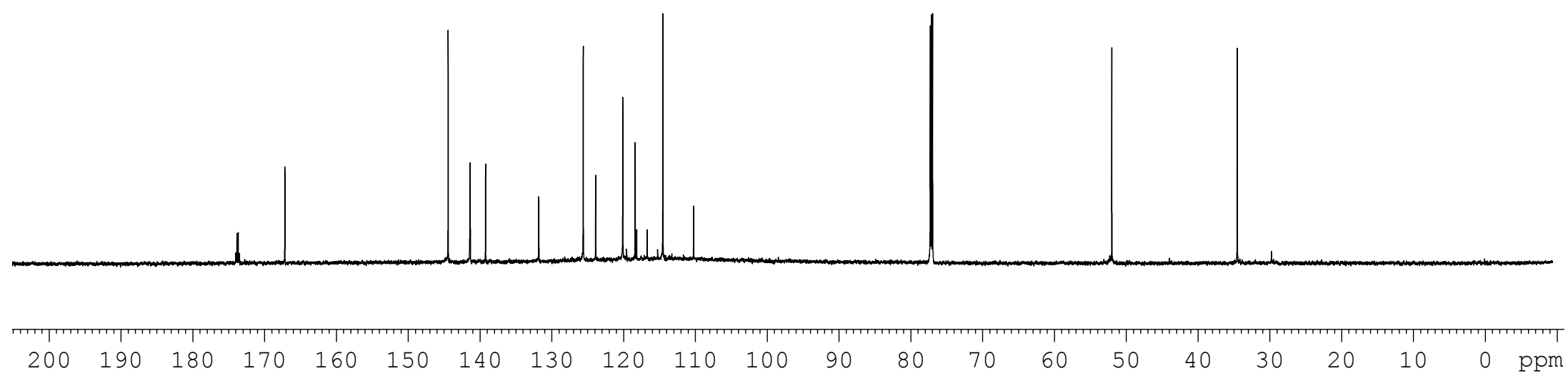




V|

LVR-06176A
樬跑

II

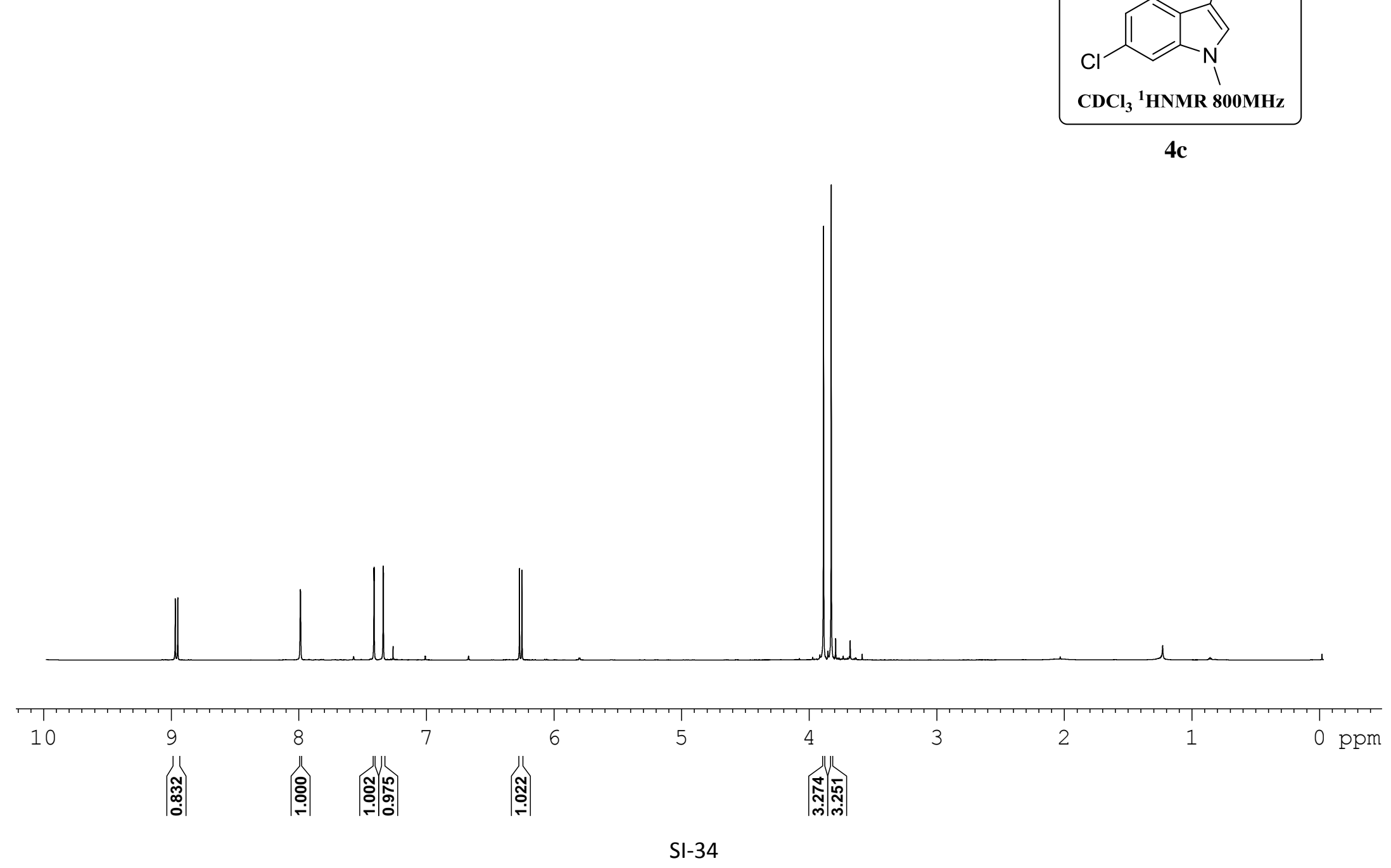




V|

LVR-06176A
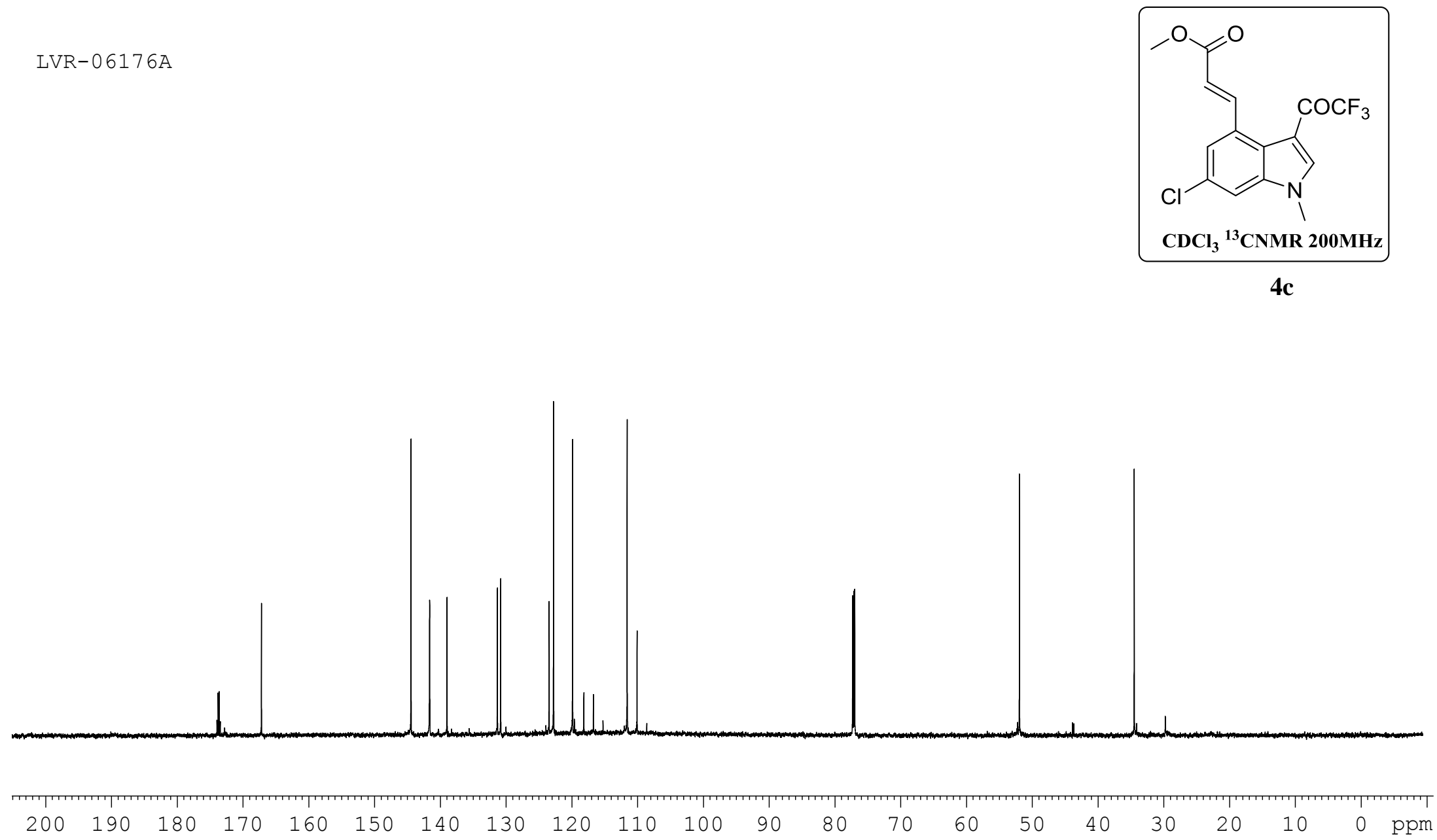
Lanke, Bettadapur, and Prabhu/Org Chem/IISc

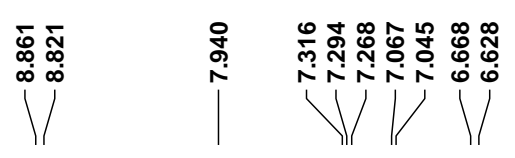

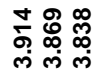

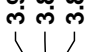

ঃั

LVR-06149B

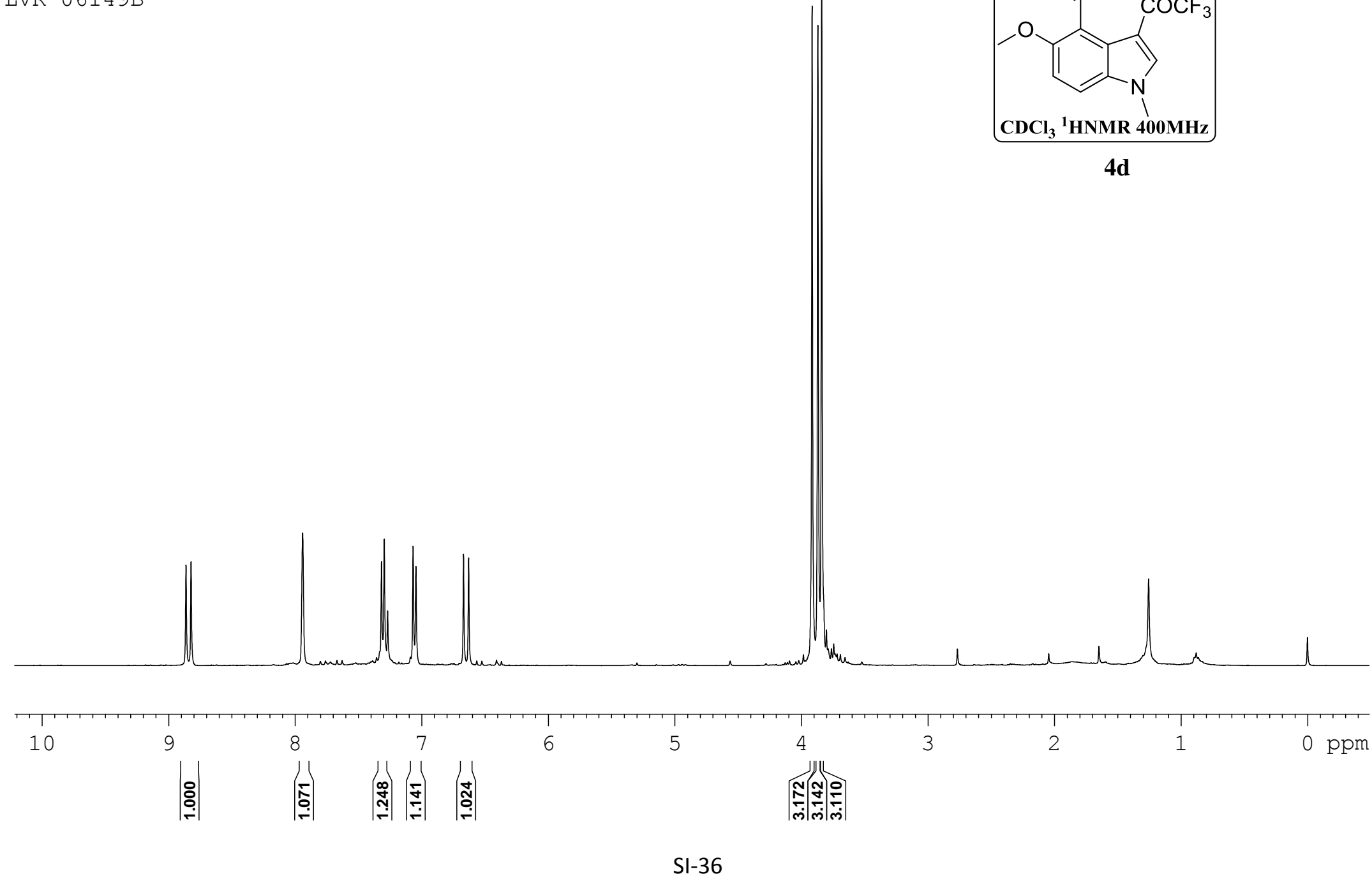




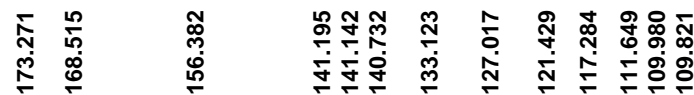 \\ I}

\section{LVR-0 6149A}

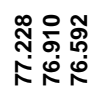

V)

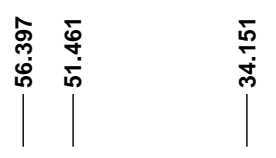

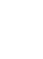
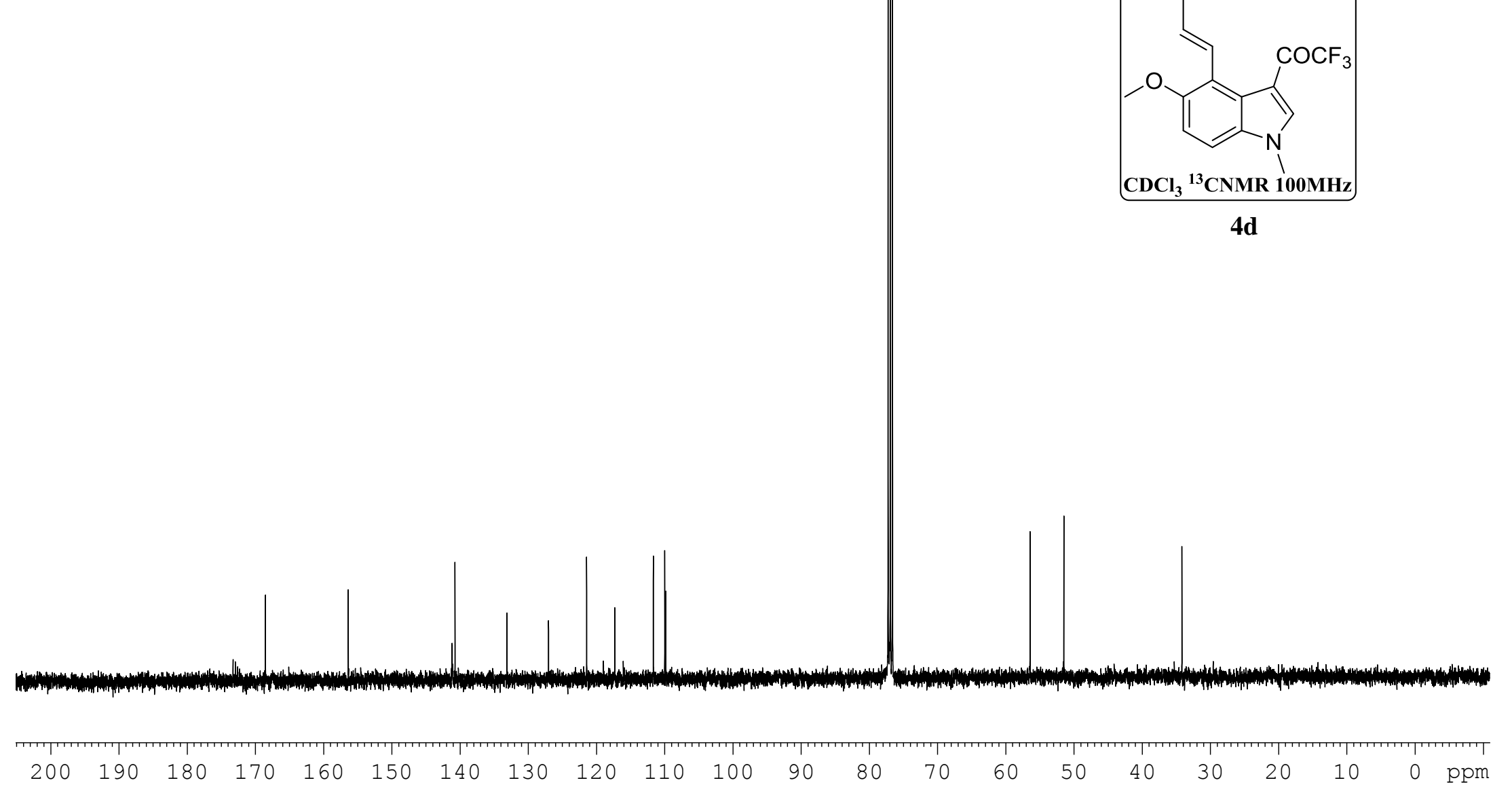


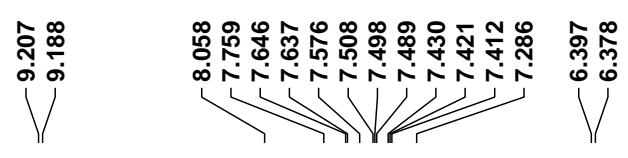

LVR-07029
今ิ

$\mid$
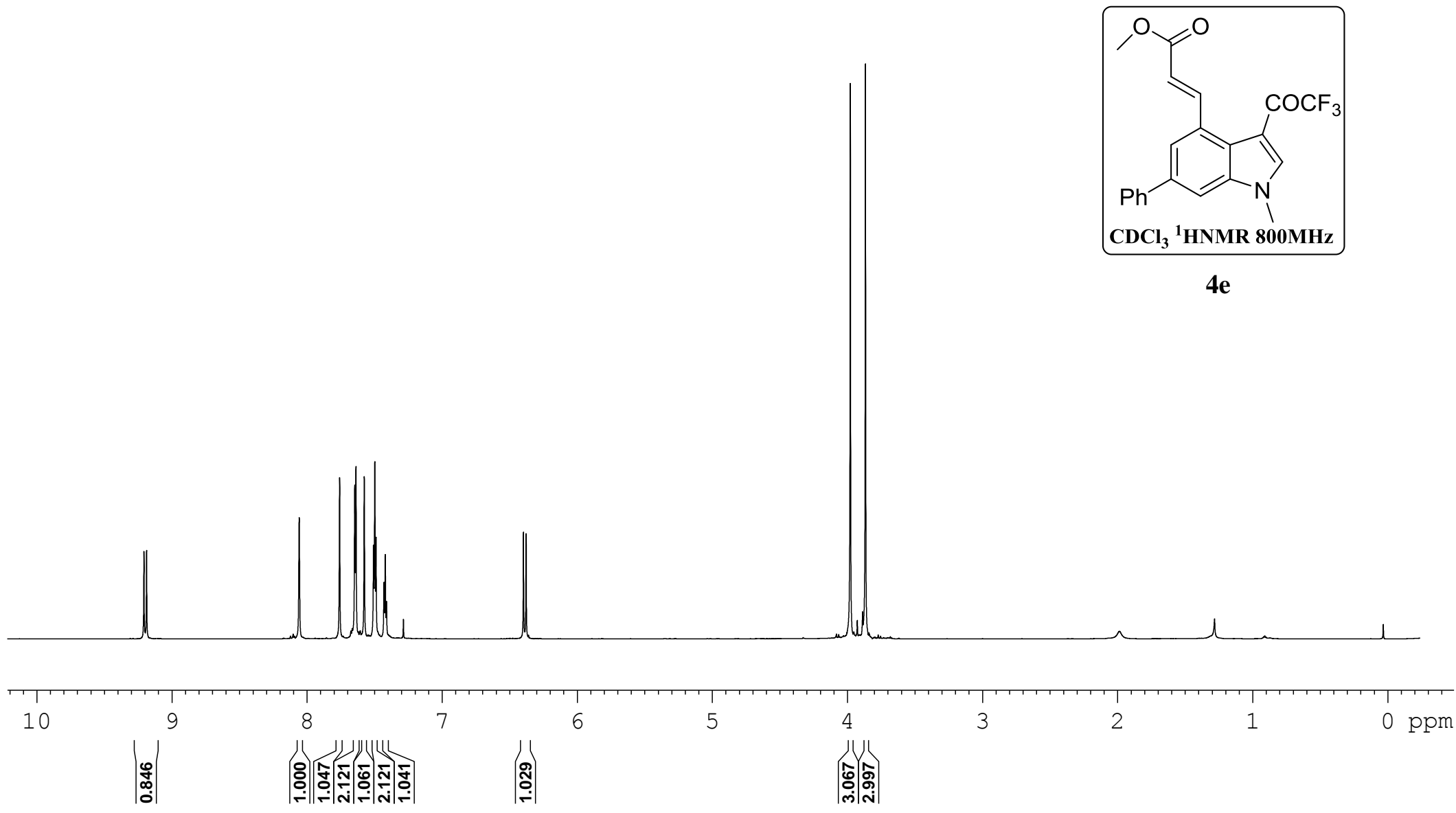


$\underbrace{2}$

LVR-07029
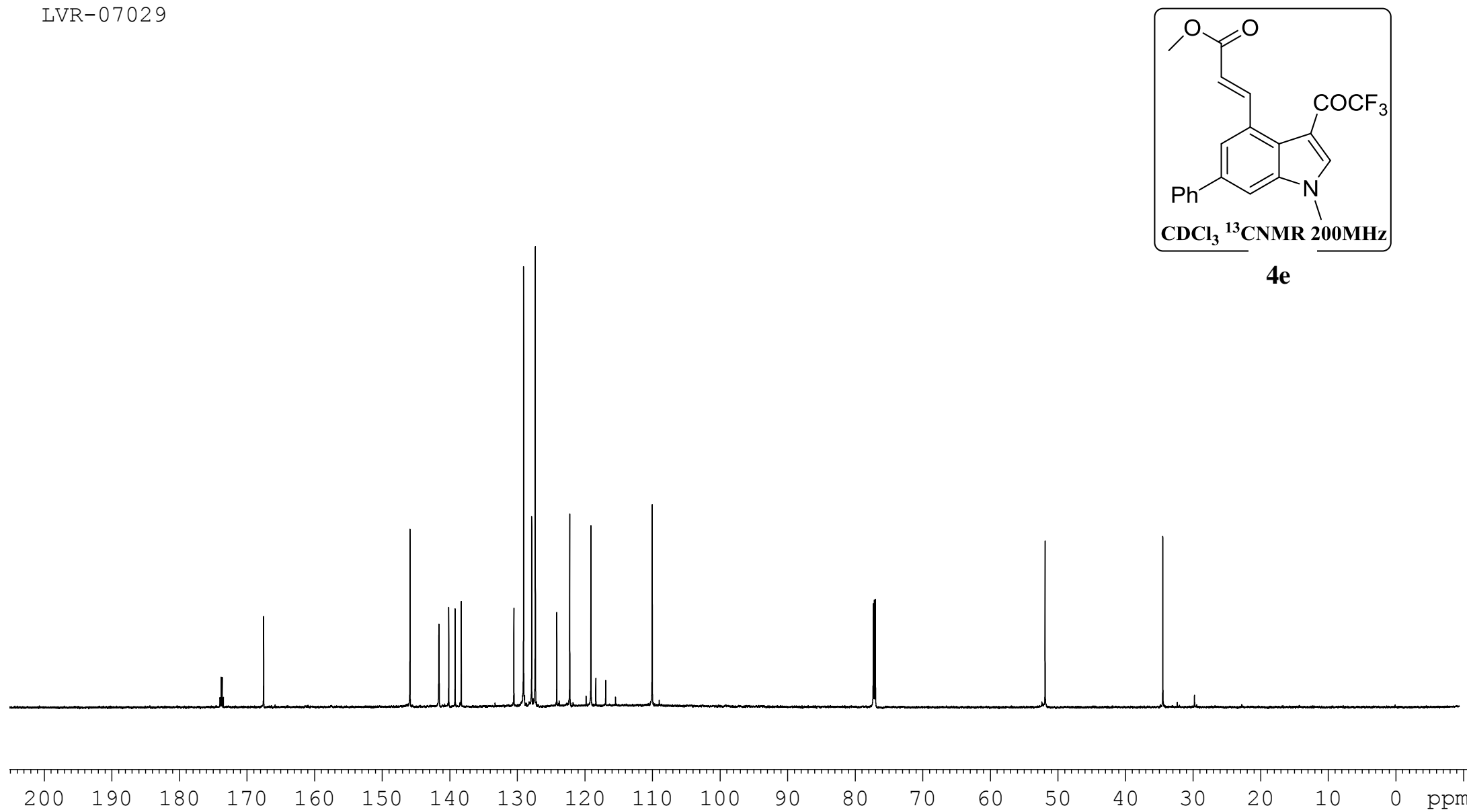
Lanke, Bettadapur, and Prabhu/Org Chem/IISC

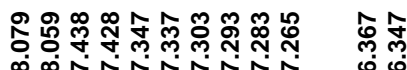 \\ VरNink \\ V}

孞志

$\left.\right|^{\infty}$

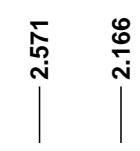

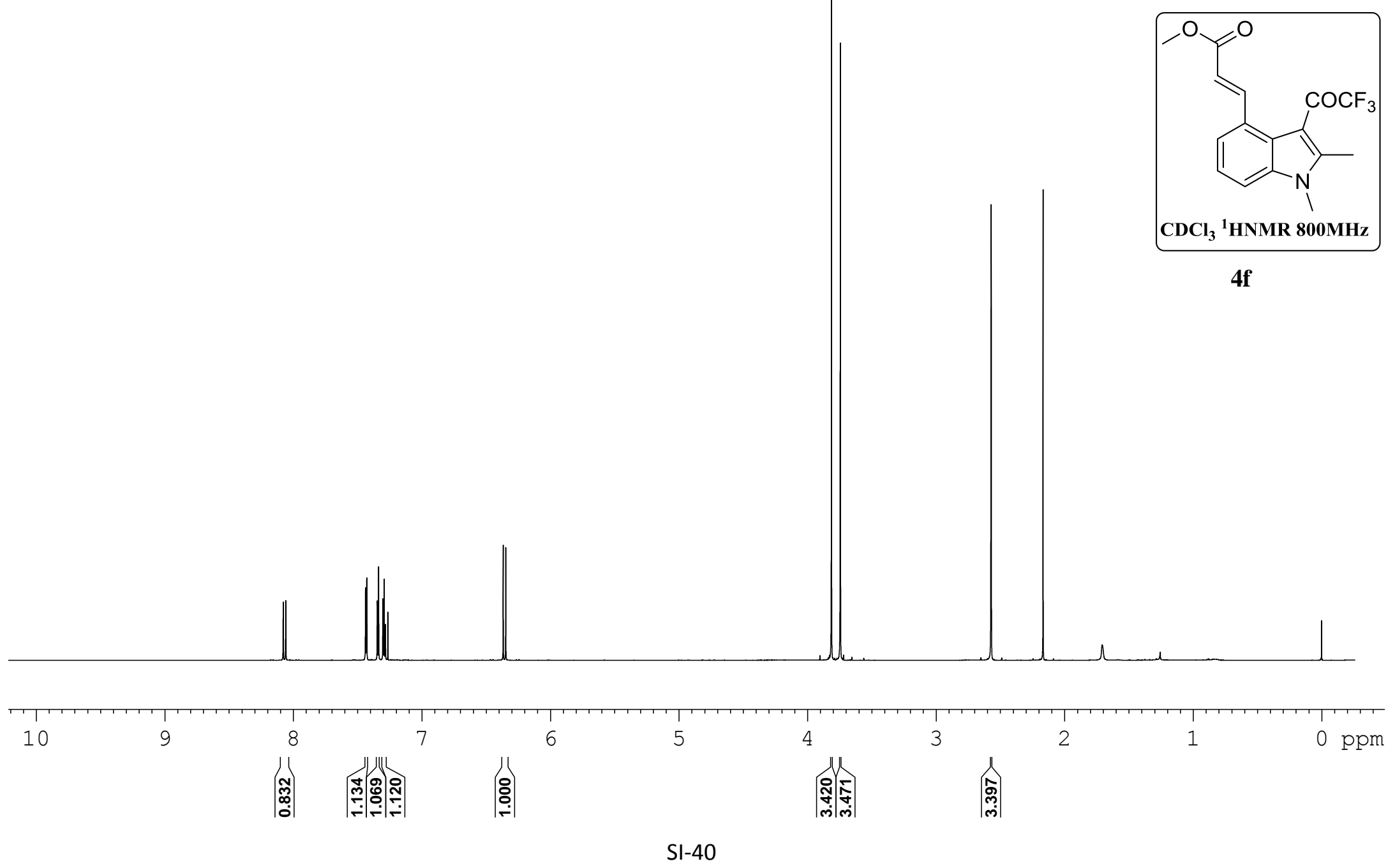

LVR-08014B 


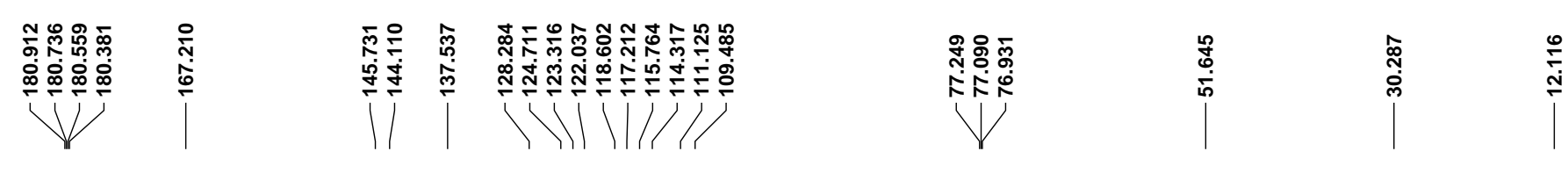

\section{LVR-0 $8014 \mathrm{~B}$}
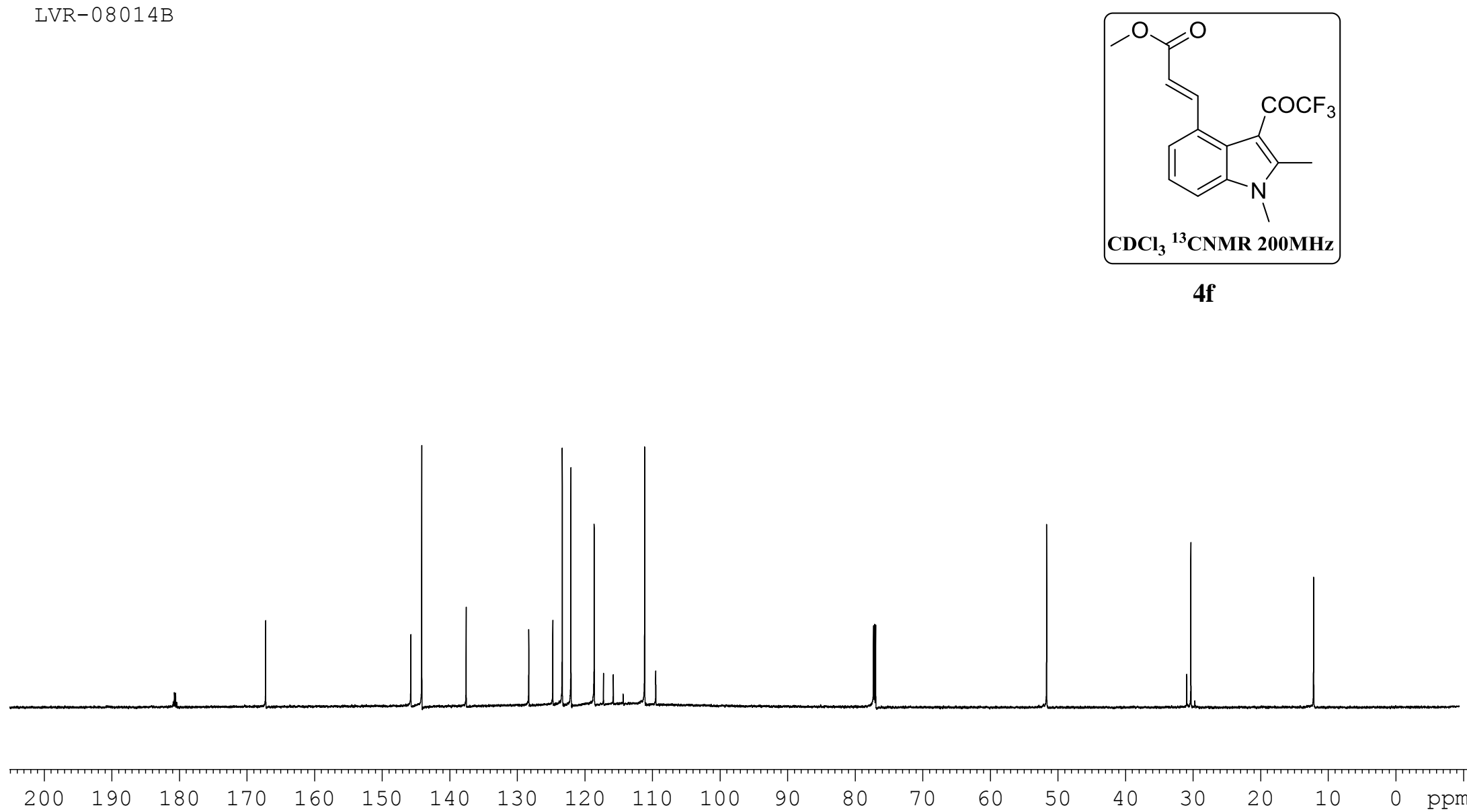


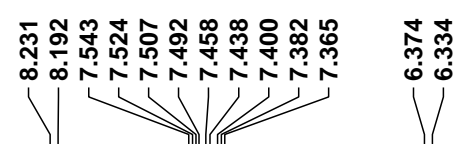

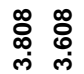

1

LVR-06160B

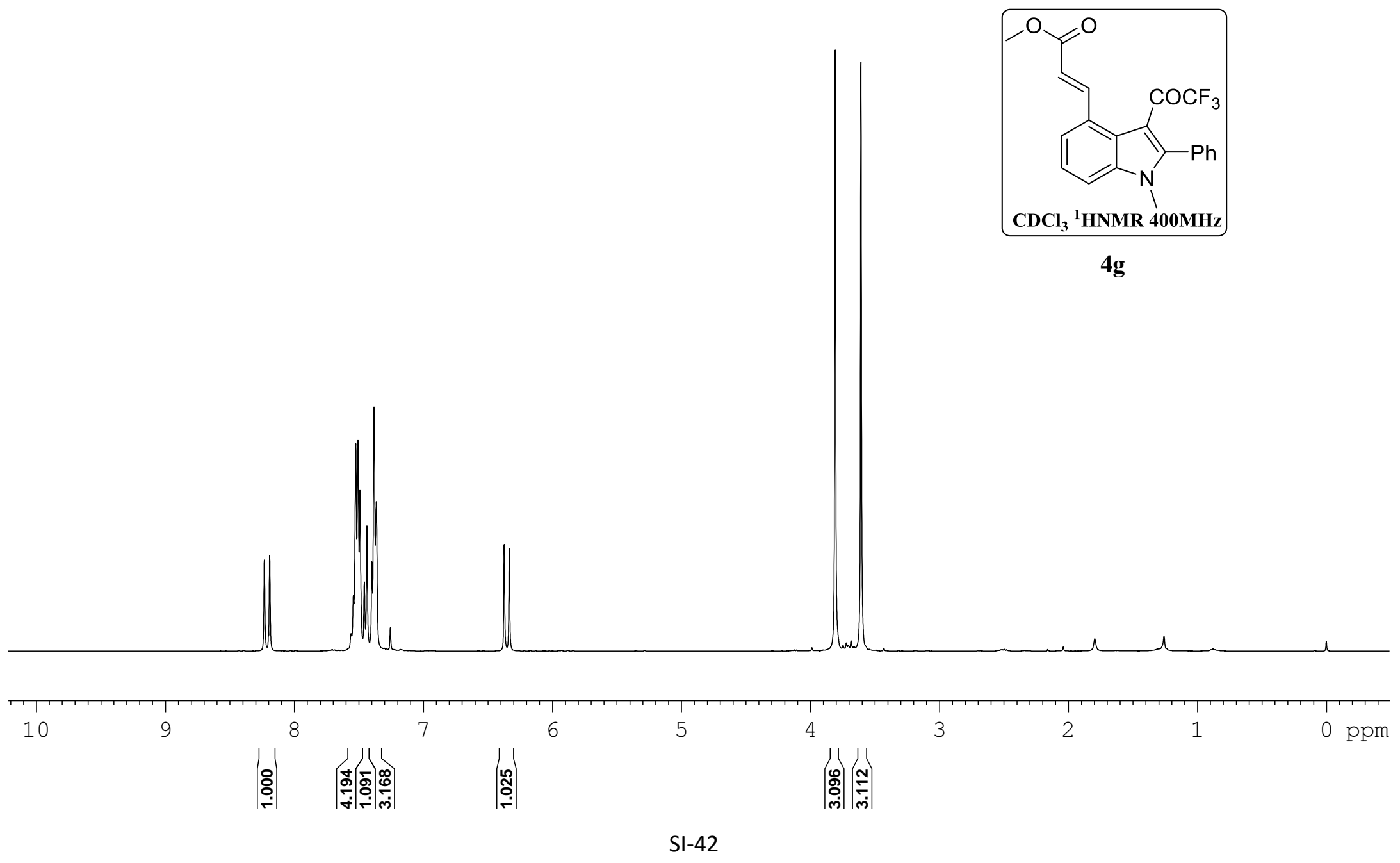




$\sum^{\mid}$

LVR-06160B

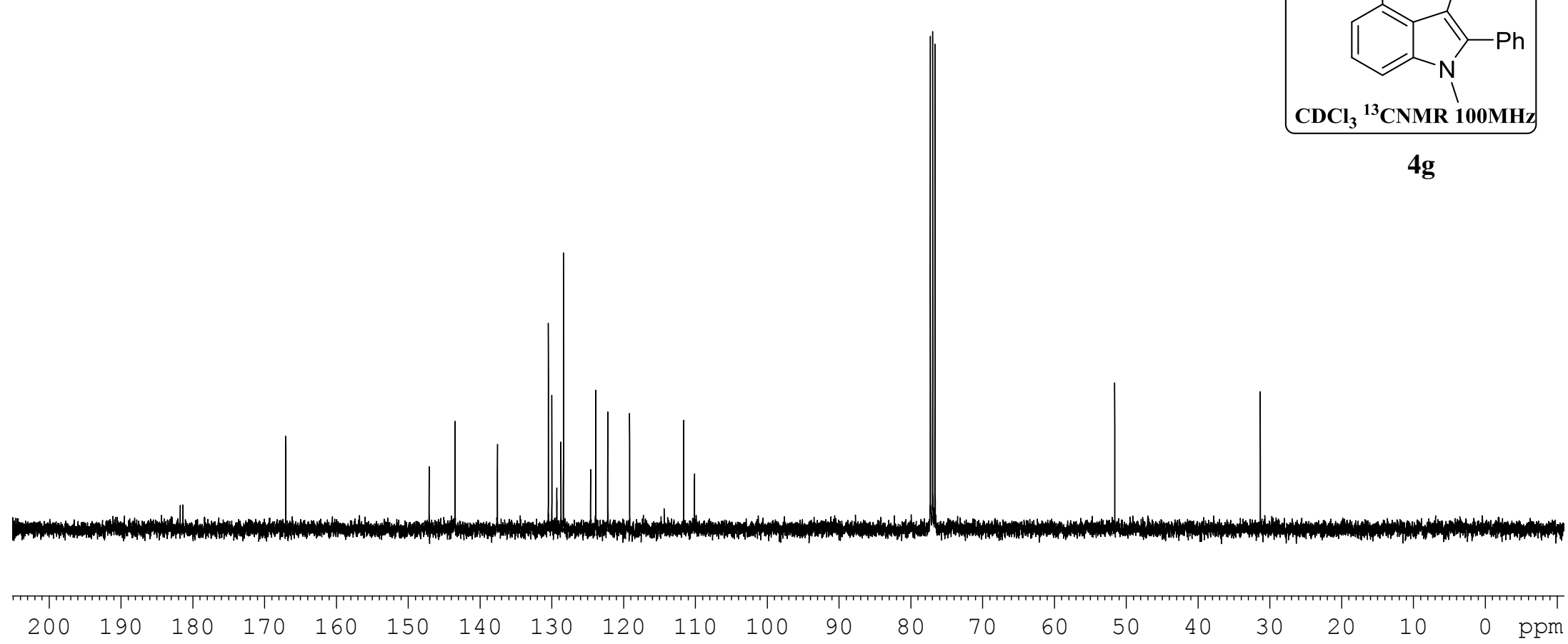




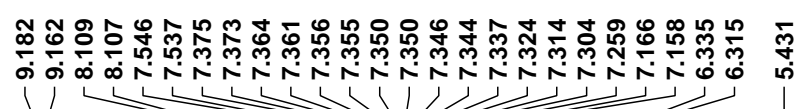

离

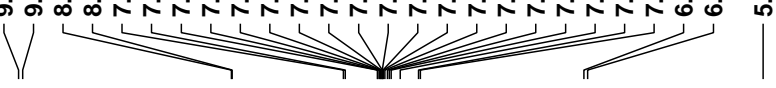

LVR-07189A

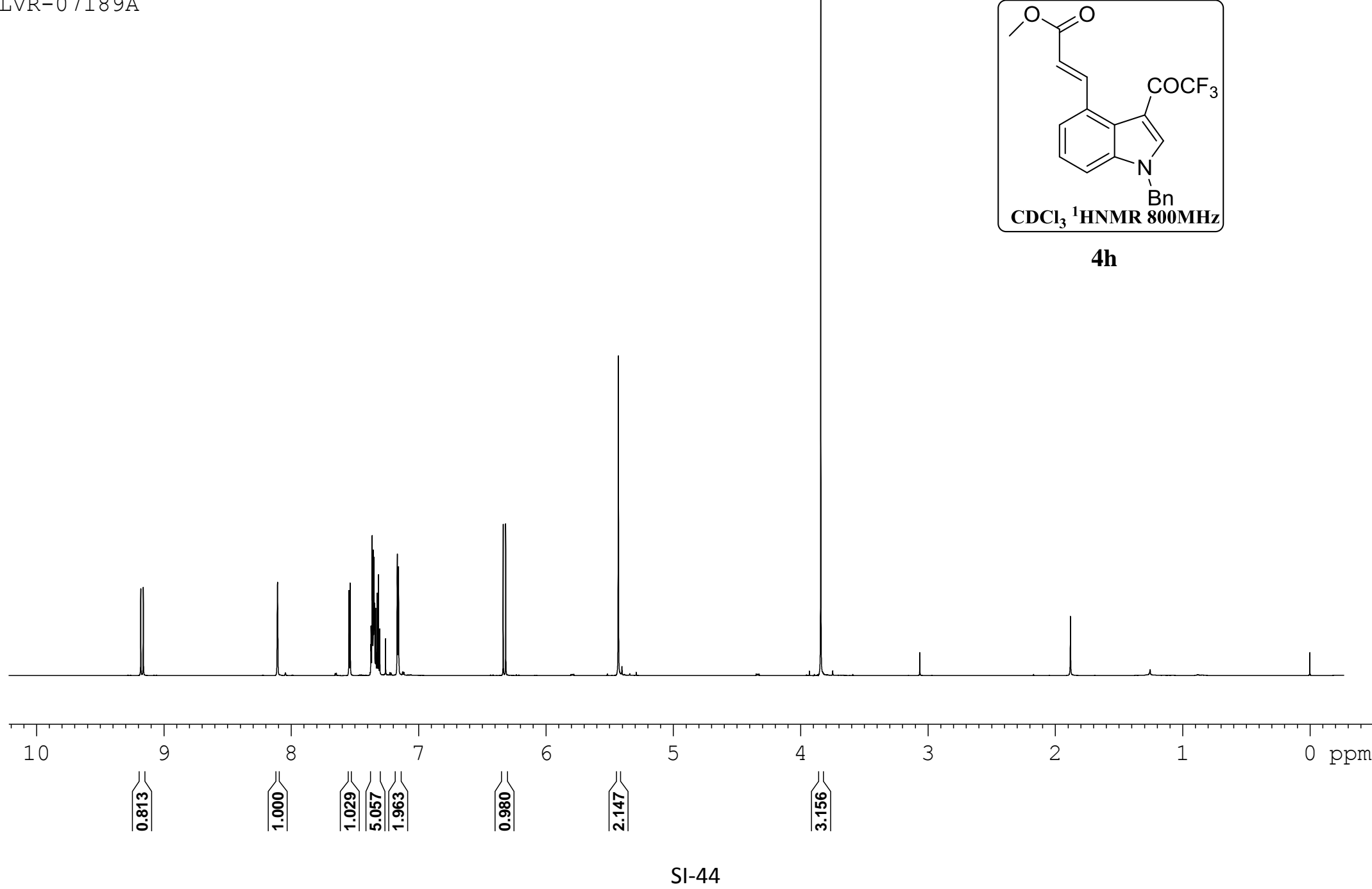




W|

LVR-07189A

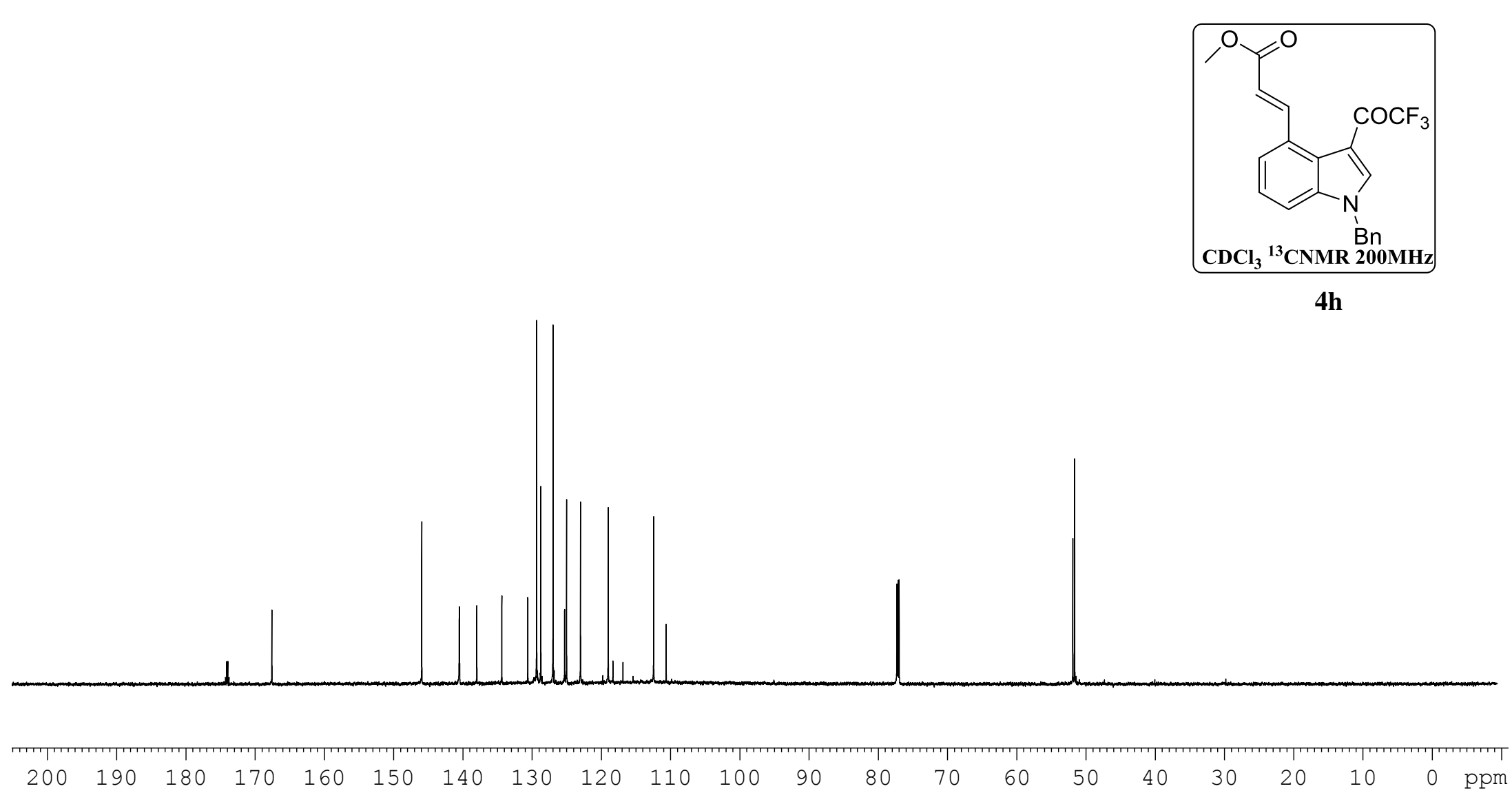




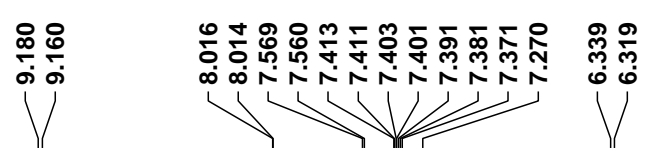

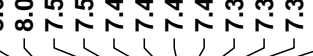

WW

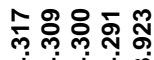

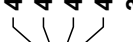

एं।

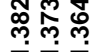

V

$4 i$

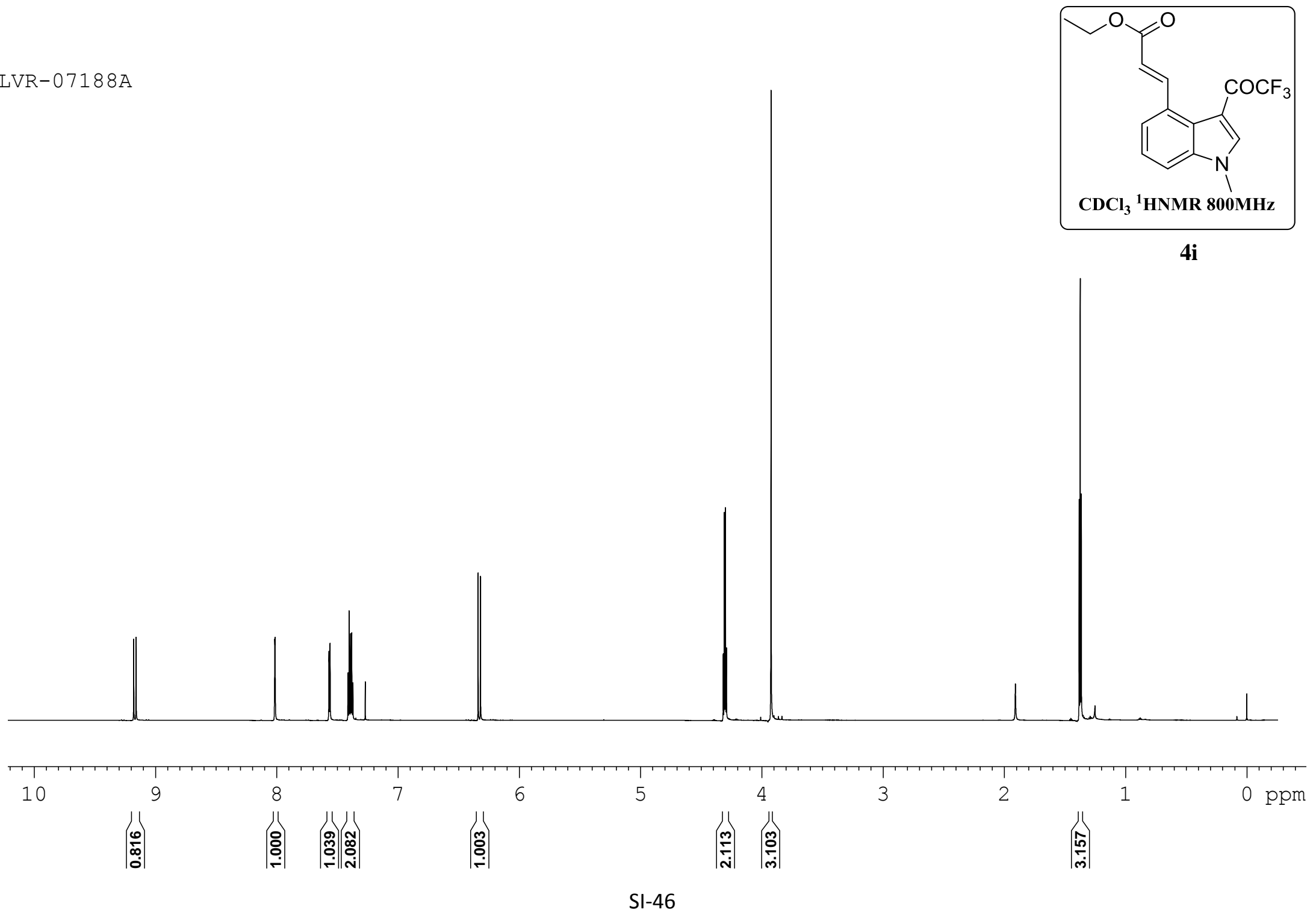

LVR-07188A 


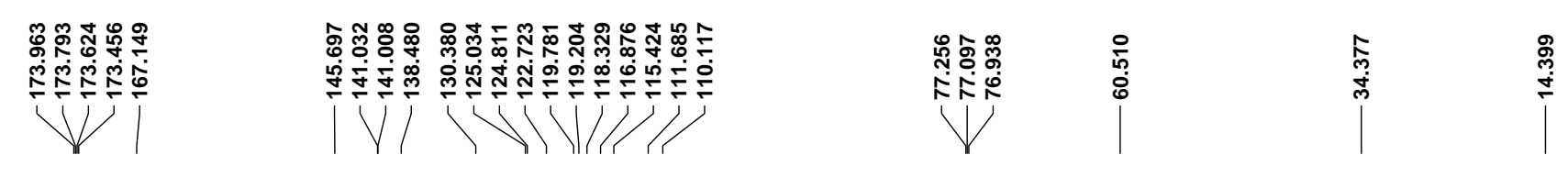

LVR-07188A

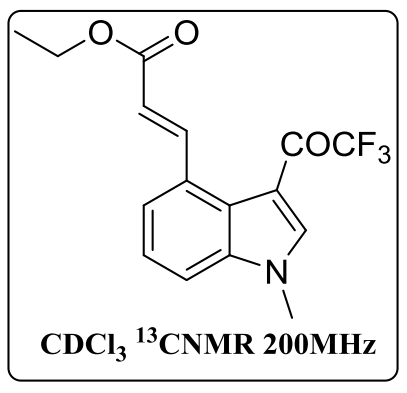

$4 \mathbf{i}$
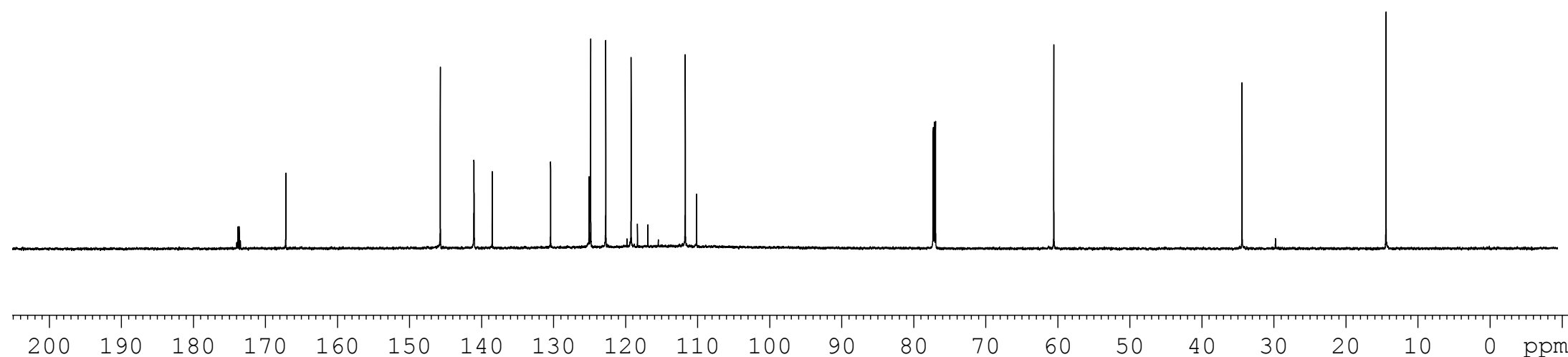


గị

\section{LVR-0 $6144 \mathrm{~B}$}

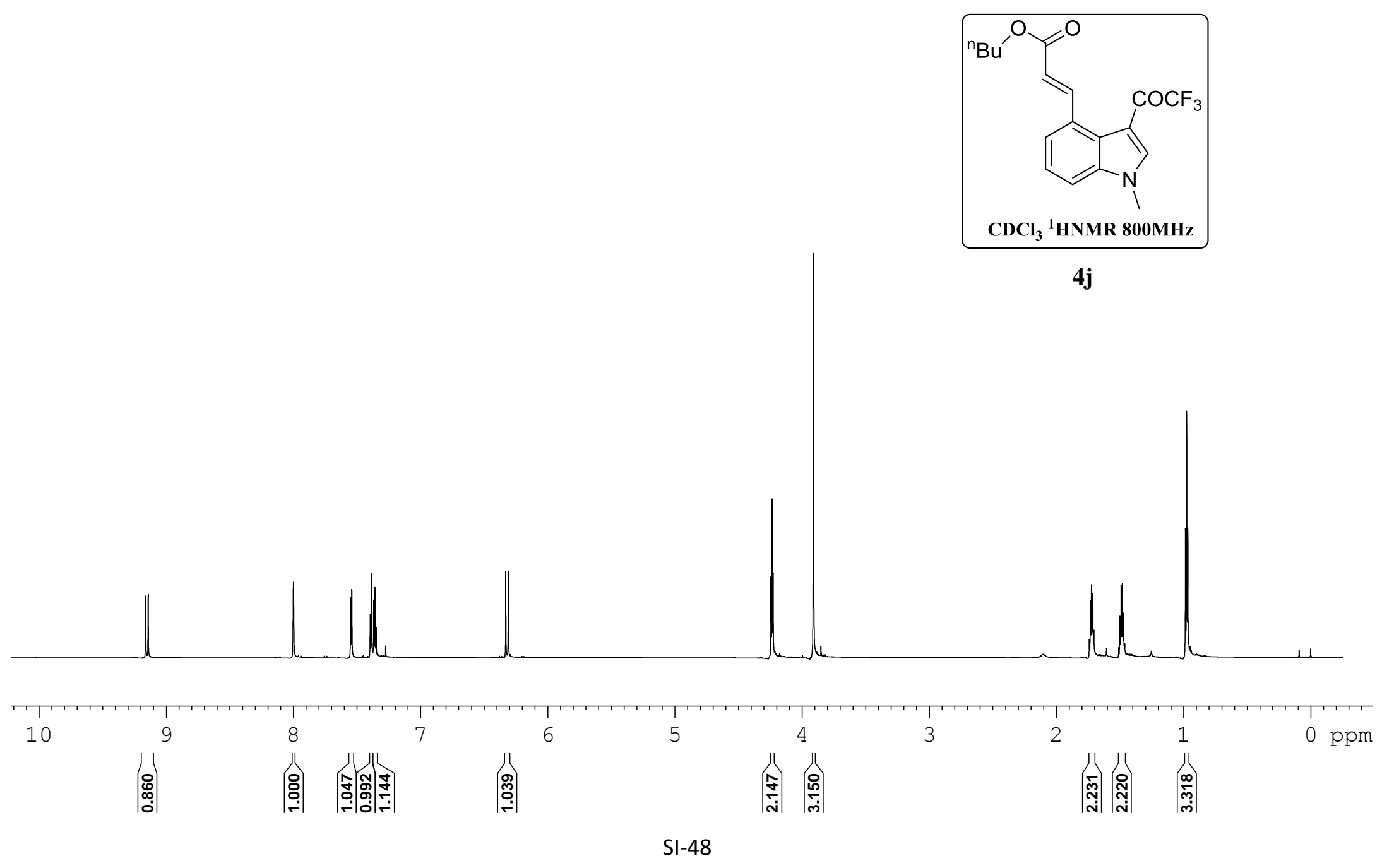




$\underbrace{\mid}$

LVR-06144B

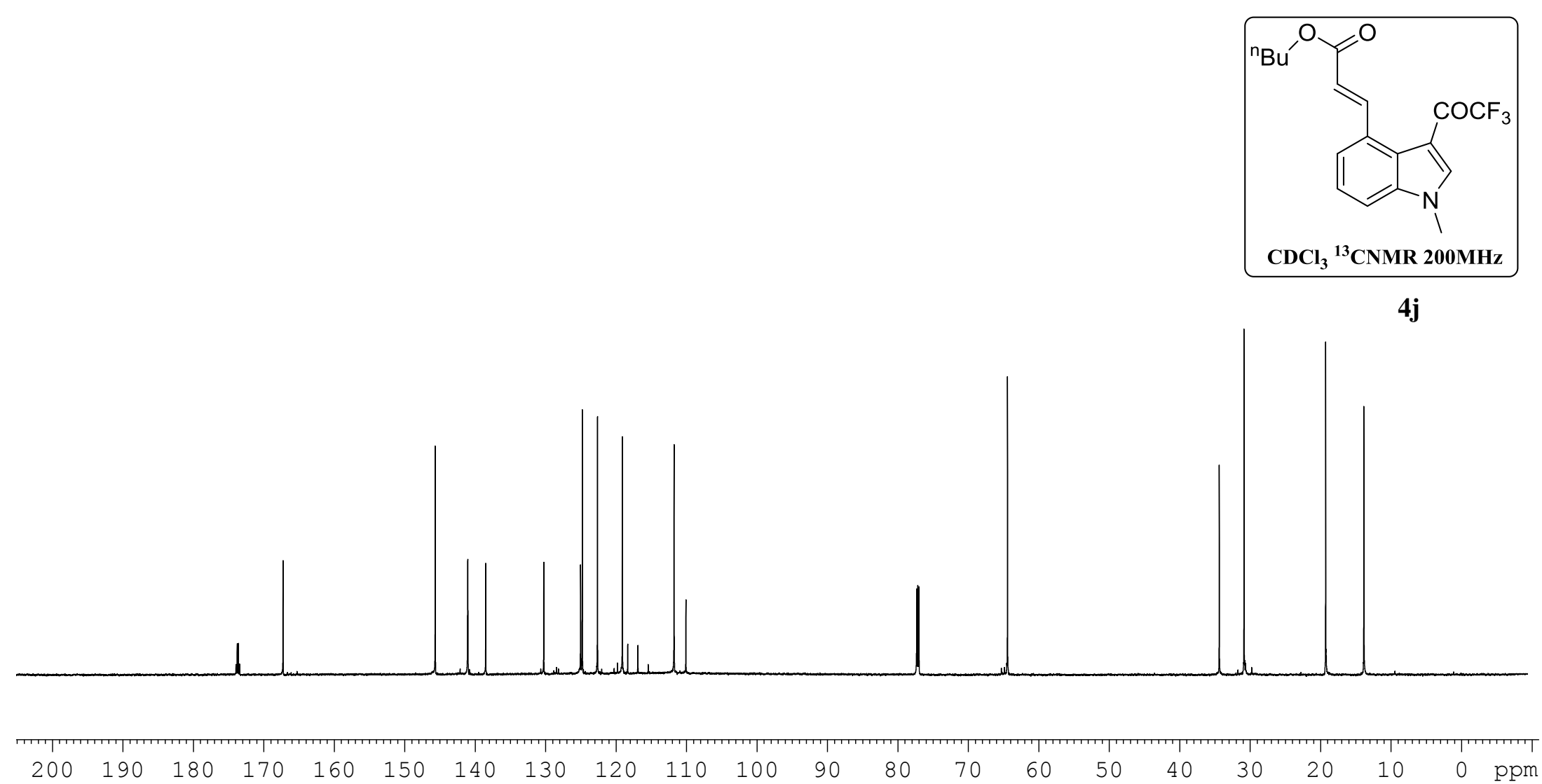


Lanke, Bettadapur, and Prabhu/Org Chem/IISc

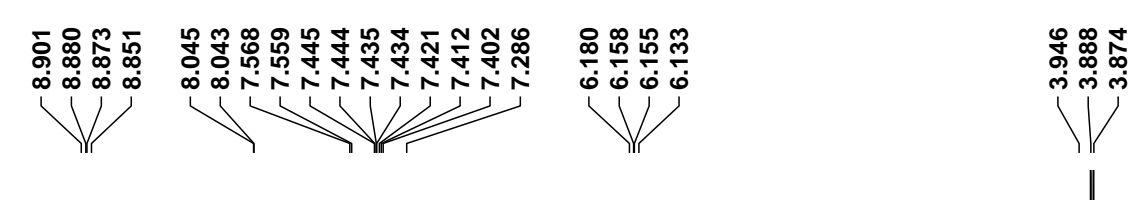

¿̊̊

LVR-06149B

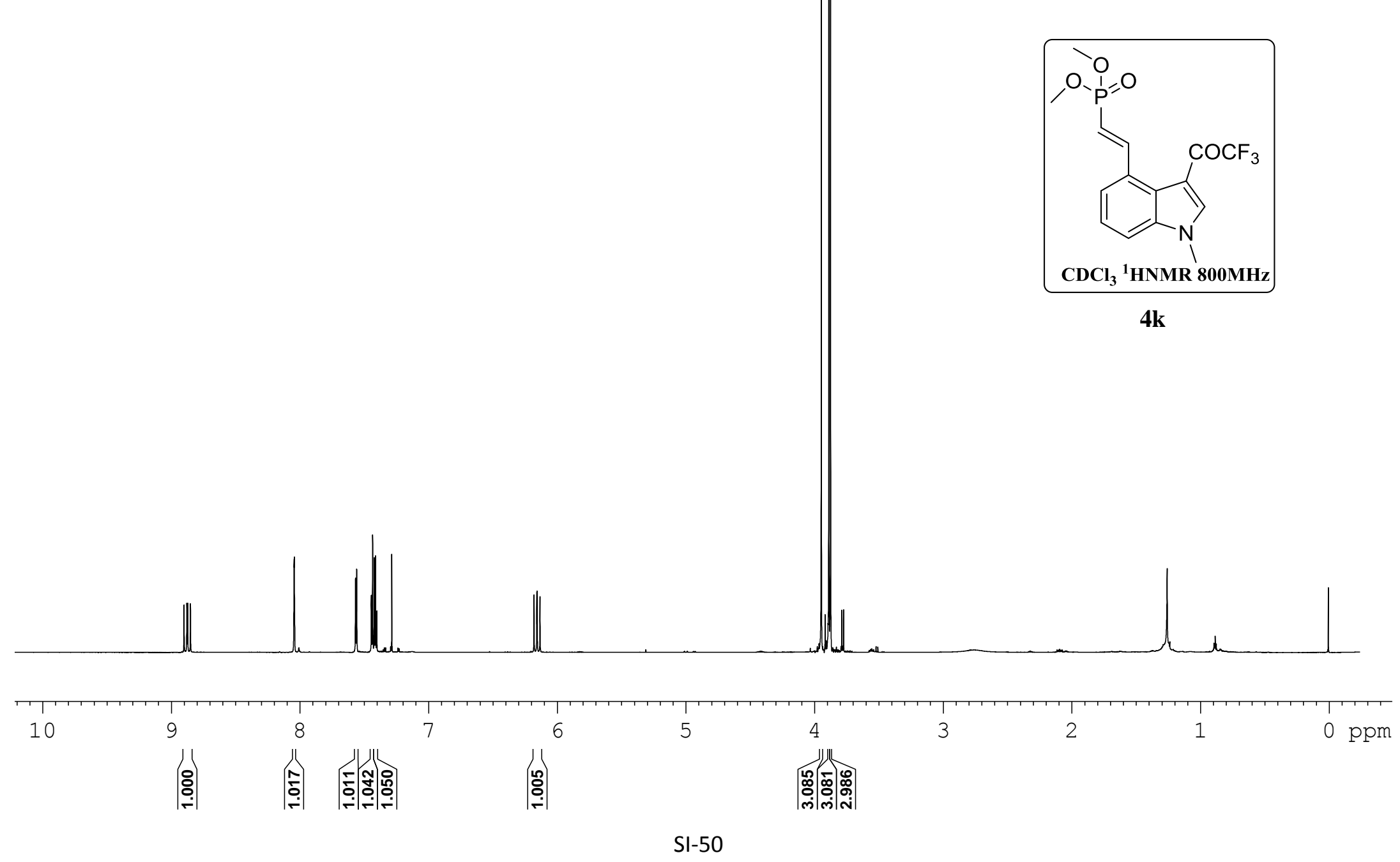




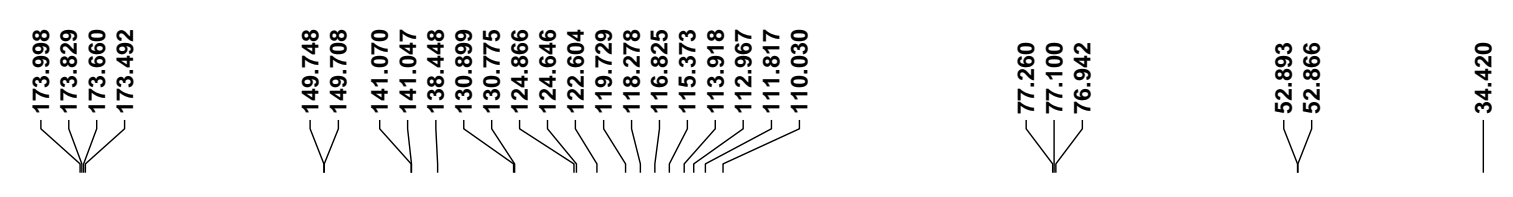

LVR-06148B

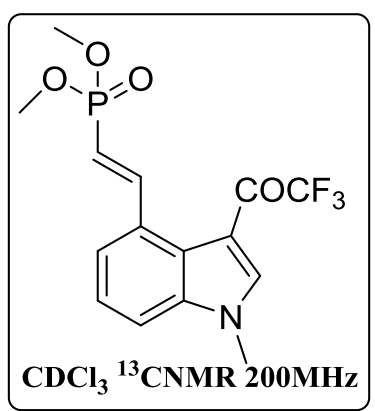

$4 k$

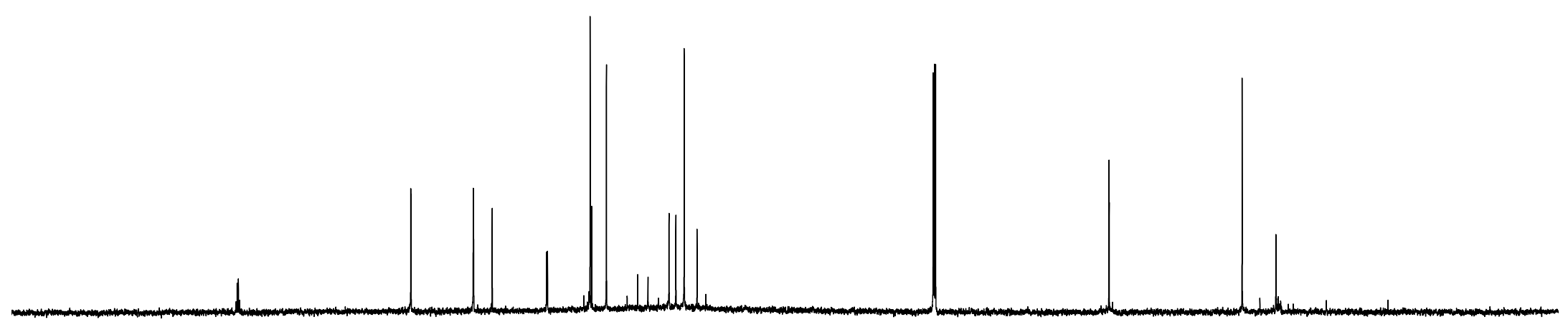




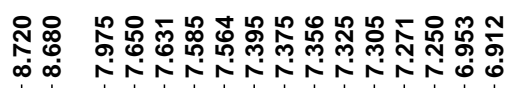

LVR-06157A
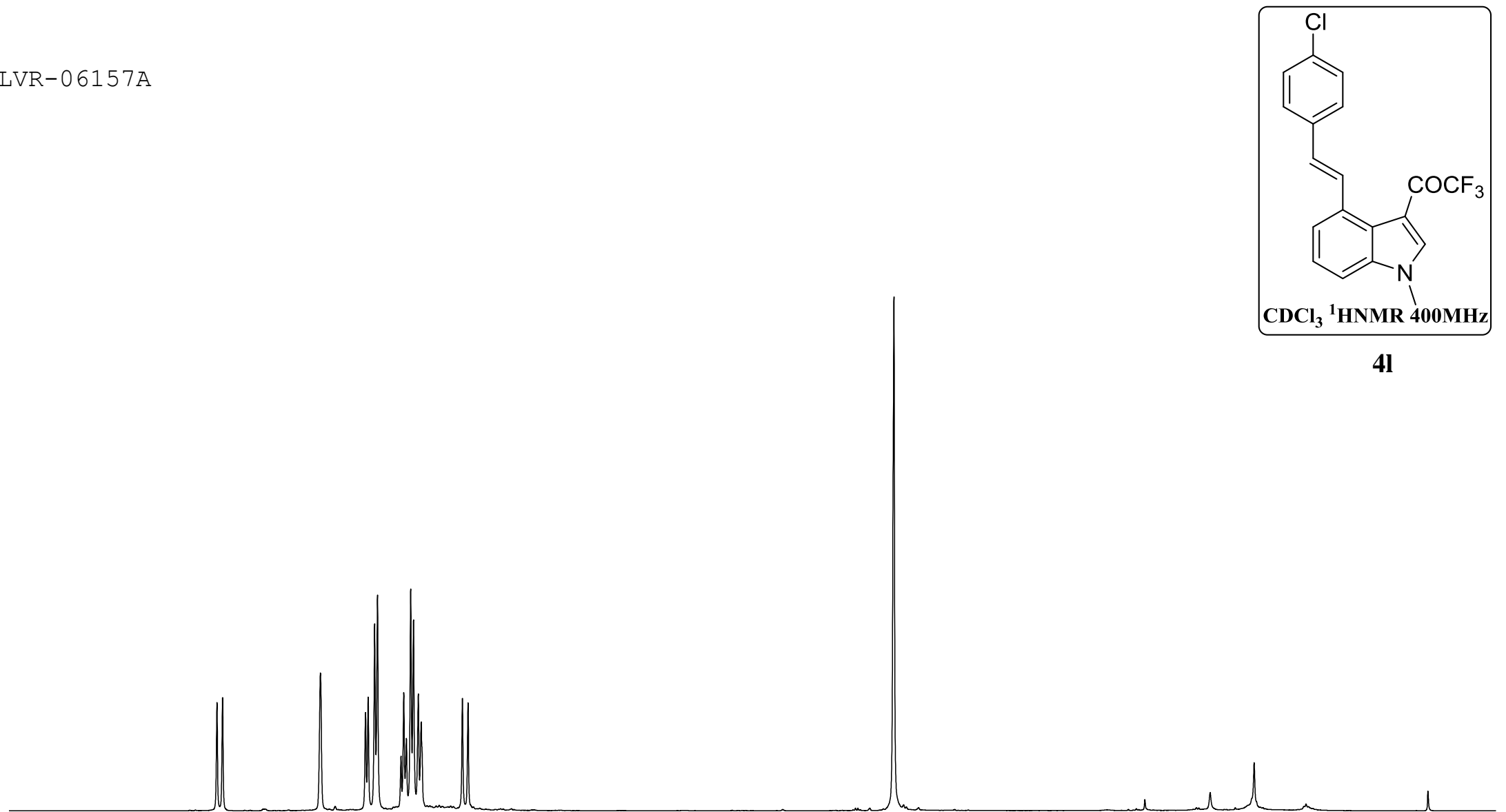

7

10

9

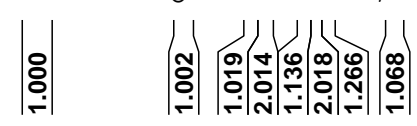
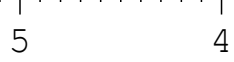
$\left|\frac{6}{\frac{6}{\infty}}\right|$ 


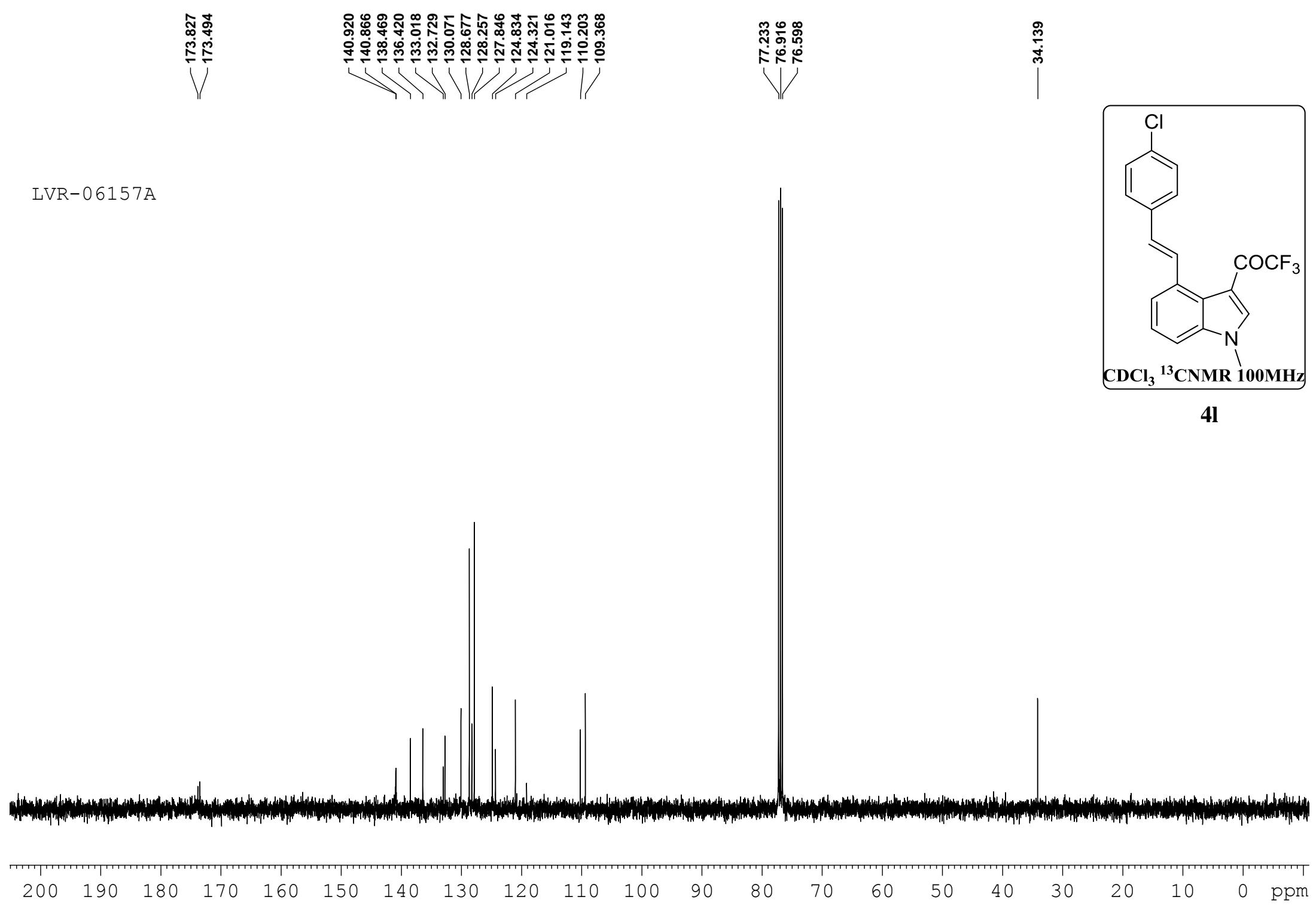




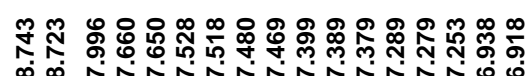

V

LVR-06161A

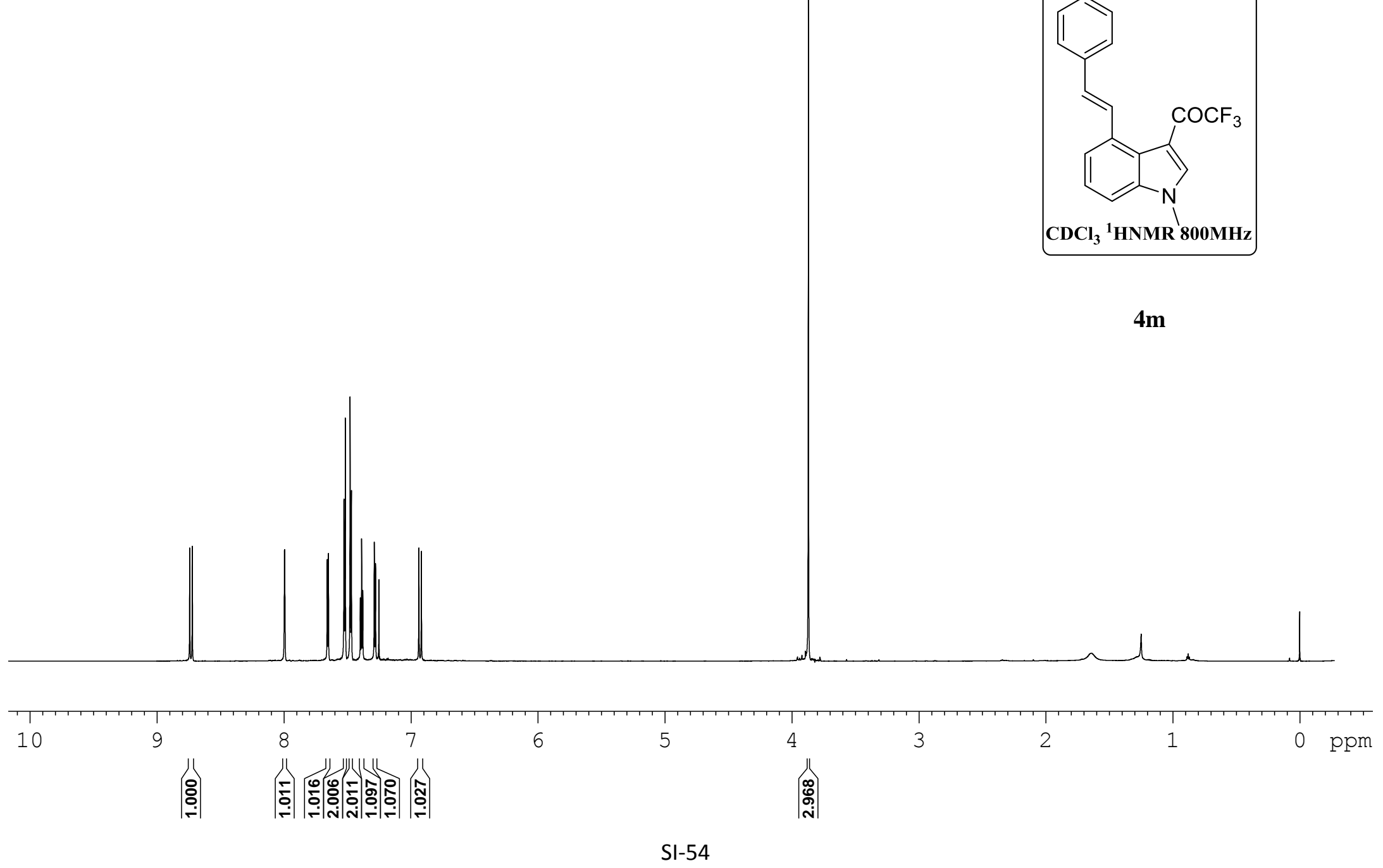




\section{In

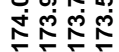 \\ V)}

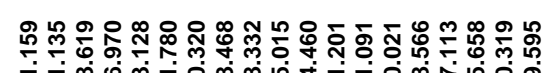

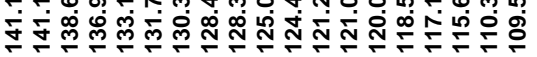

WN W VIII

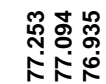

V

芯

1

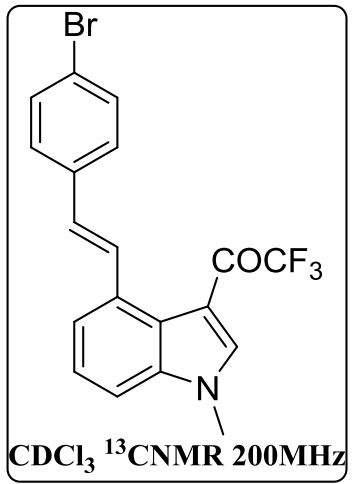

$4 m$

\section{LVR-0 6161A}

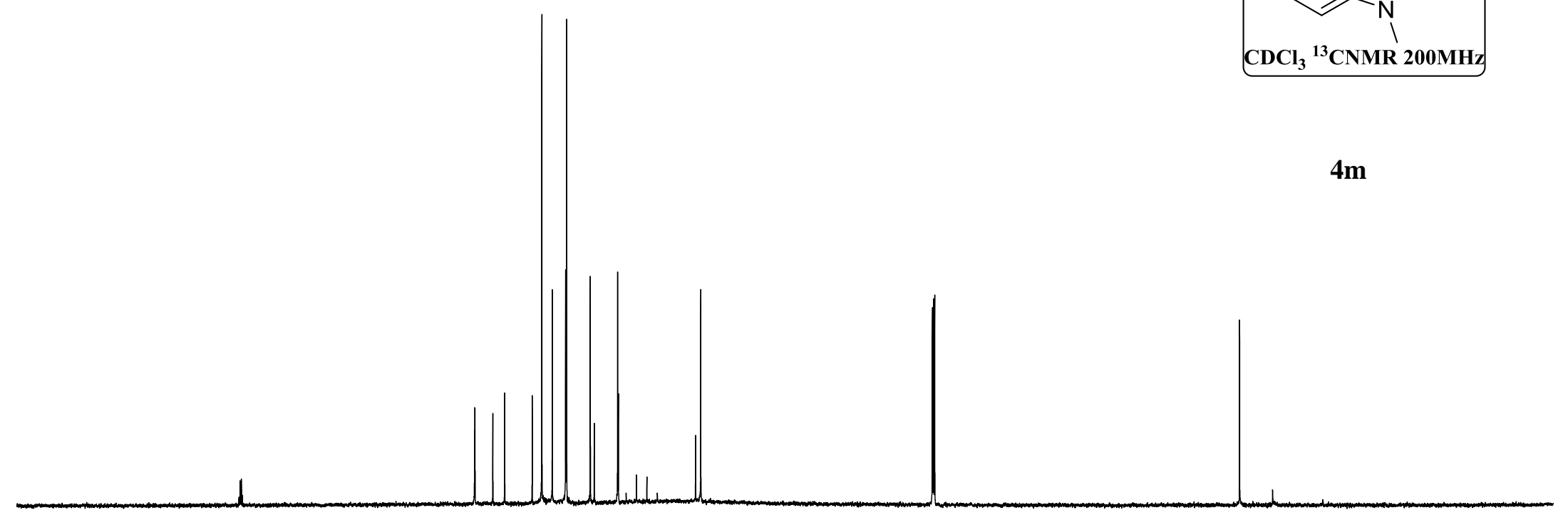




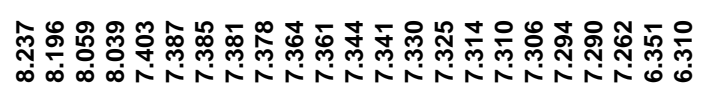

ता

总离

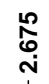

ঃั

LVR-06116

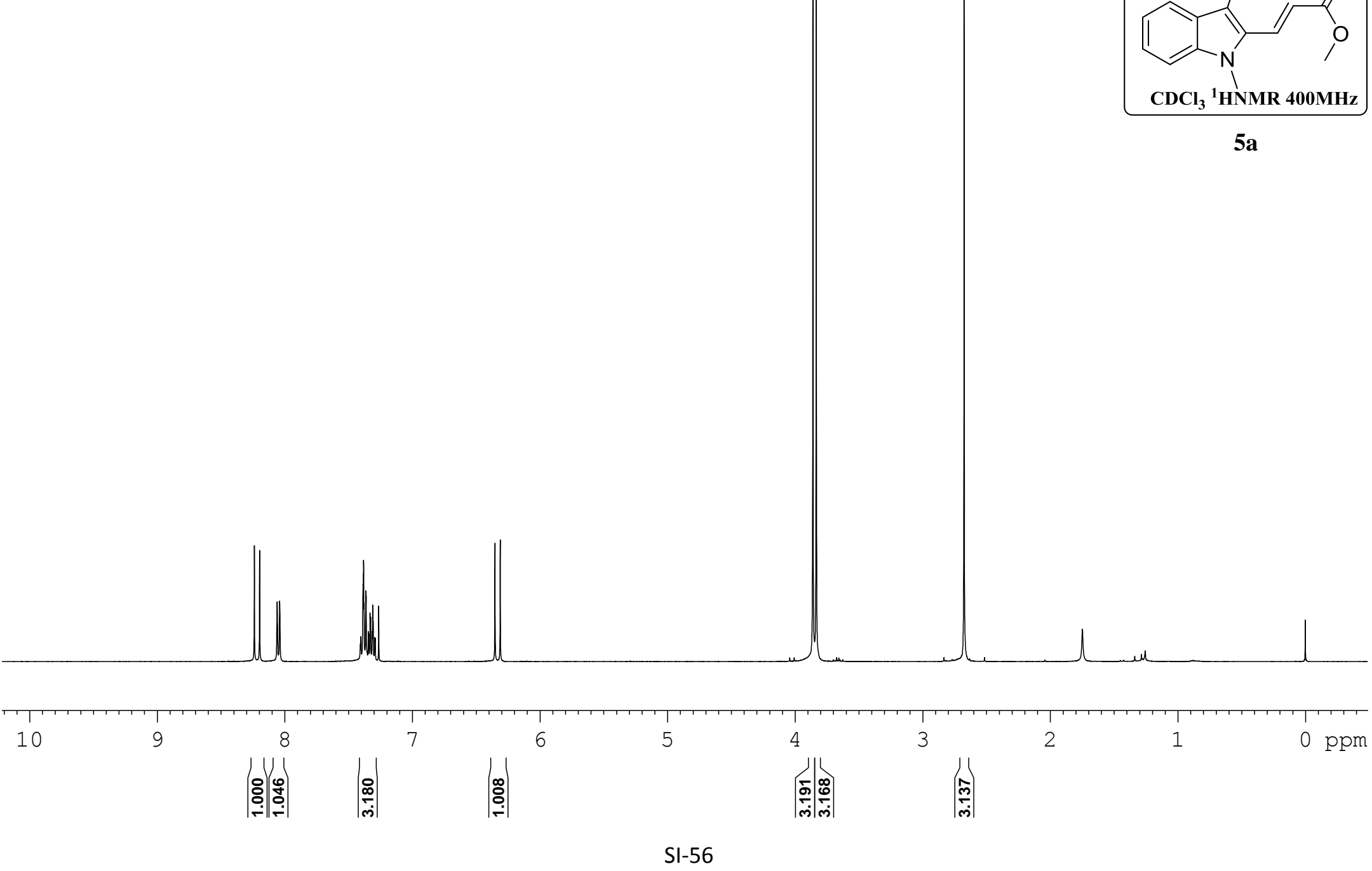




|

LVR-06116

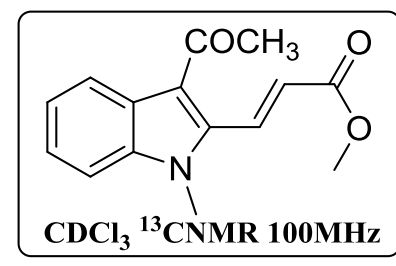

$5 \mathbf{a}$
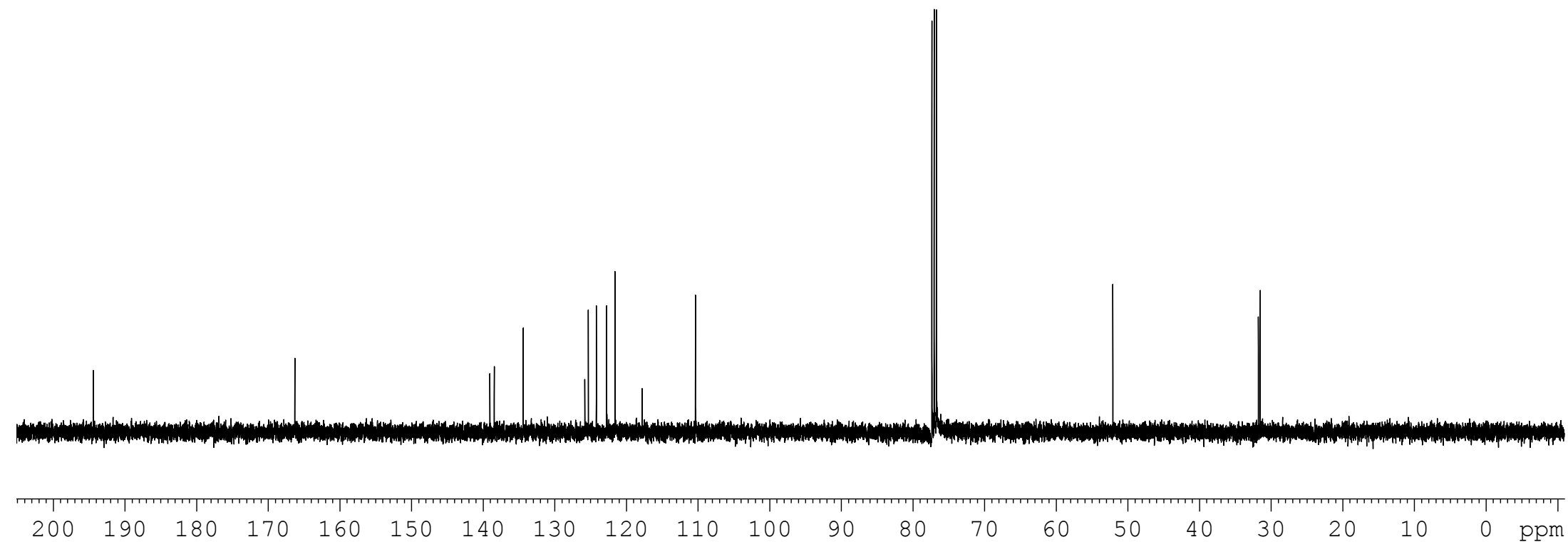
Lanke, Bettadapur, and Prabhu/Org Chem/IISc

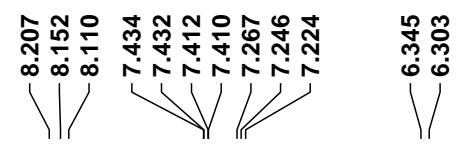

LVR-07076A

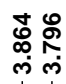

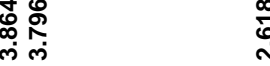

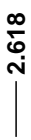

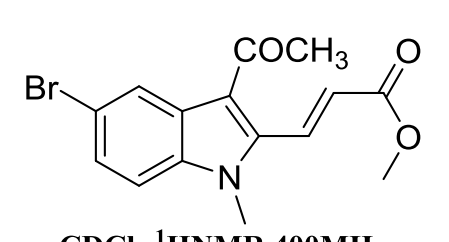

$\mathrm{CDCl}_{3}{ }^{1} \mathrm{HNMR}$ 400MHz

$5 \mathbf{b}$

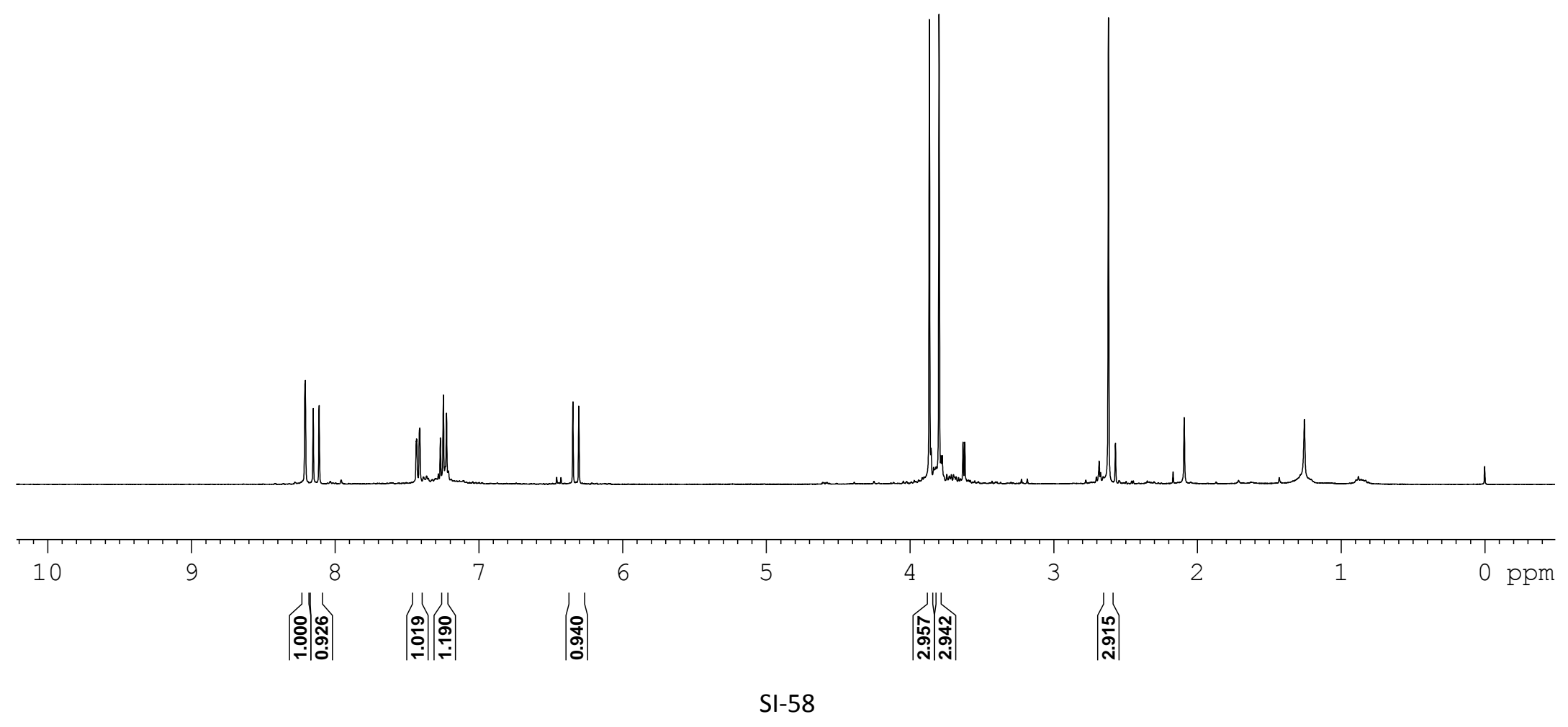




|

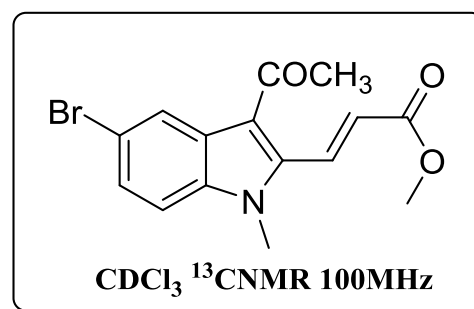

$5 \mathbf{b}$

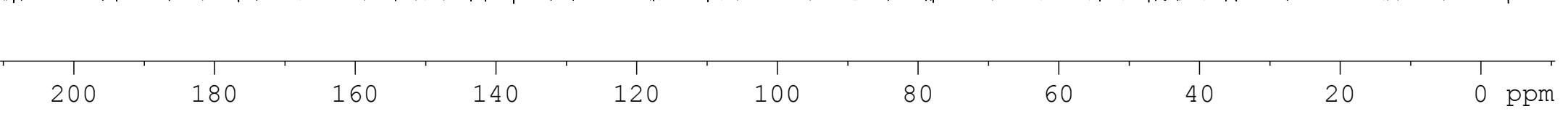

SI-59 
Lanke, Bettadapur, and Prabhu/Org Chem/IISc

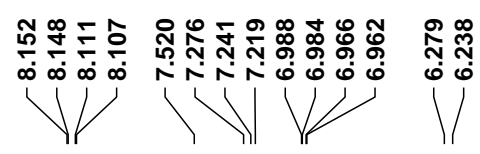

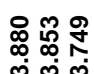

윰

ঃั

LVR-07006B

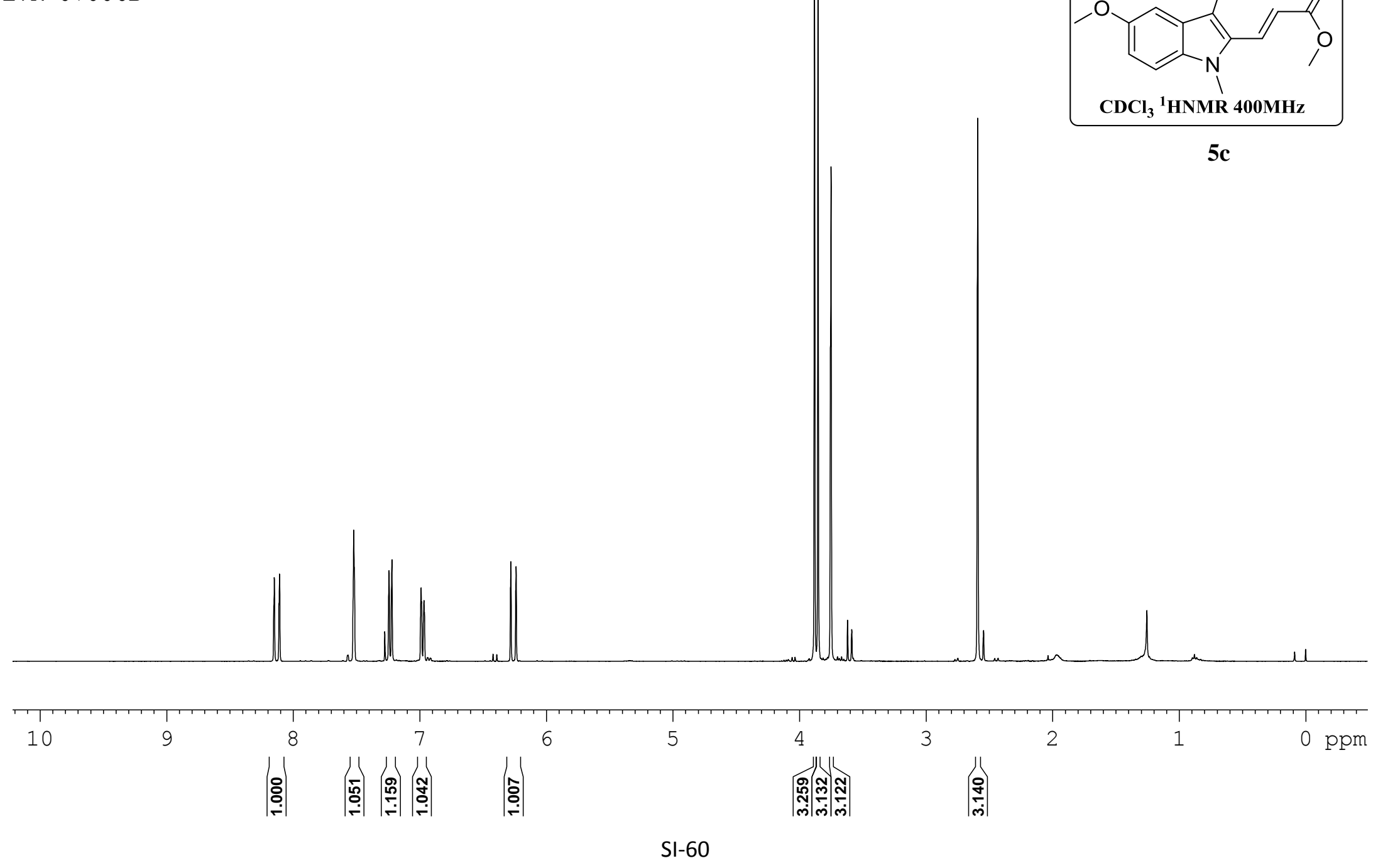




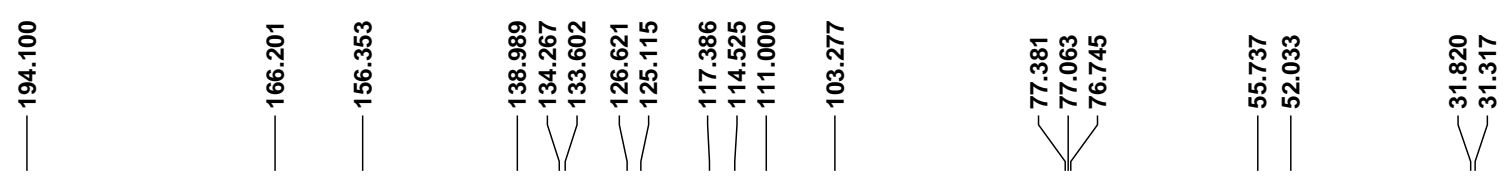

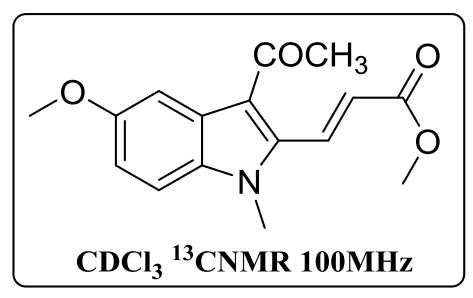

$5 \mathbf{c}$

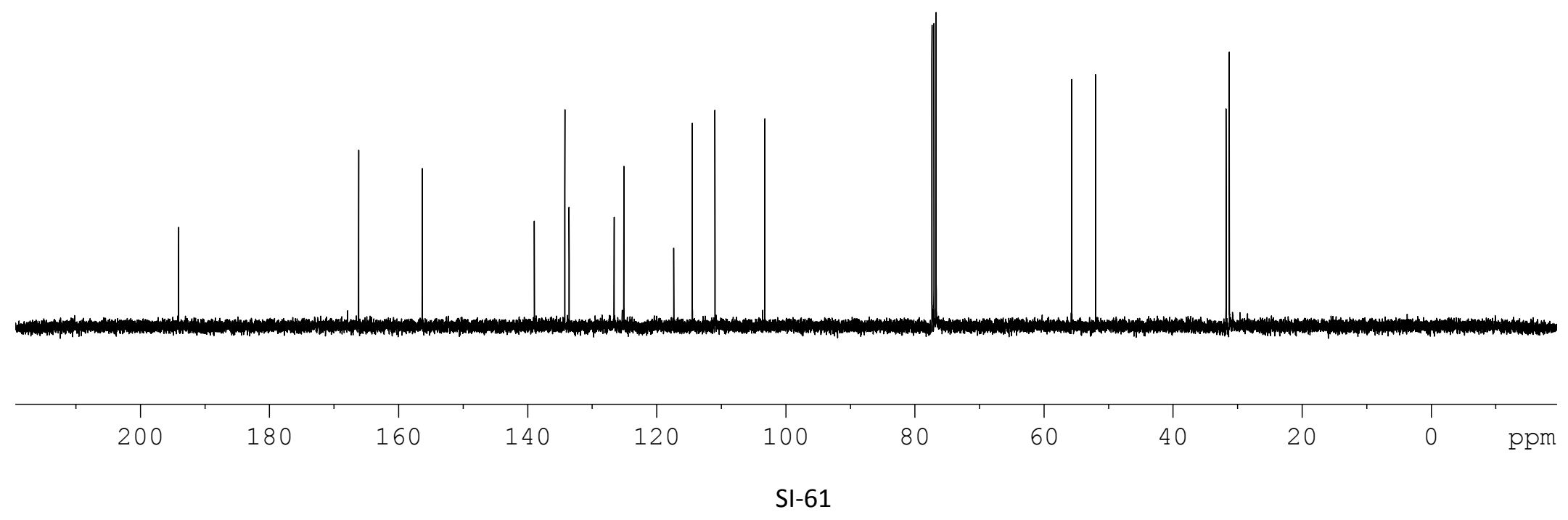




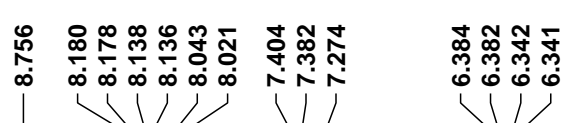

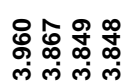

ำ

i̊

LVR-08012B

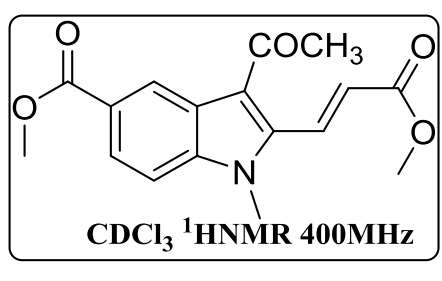

5d
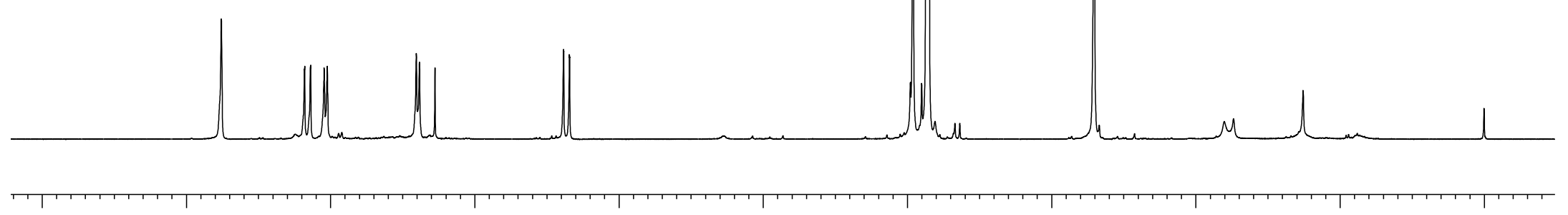

10

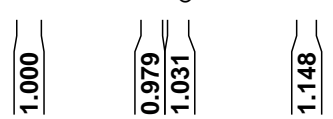

$\left|\begin{array}{l}\mid \\ 0 \\ 0 \\ \hdashline\end{array}\right|$

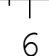

5

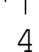
4

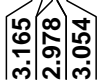

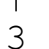

2

1

0 ppm

$\left|\begin{array}{c}\mathfrak{N} \\ \text { ลิ }\end{array}\right|$ 


|

LVR-08012B

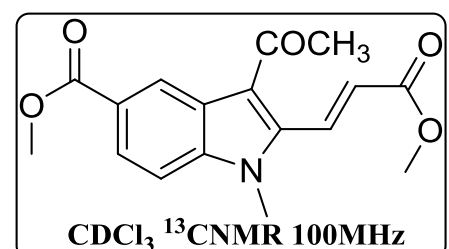

$5 d$

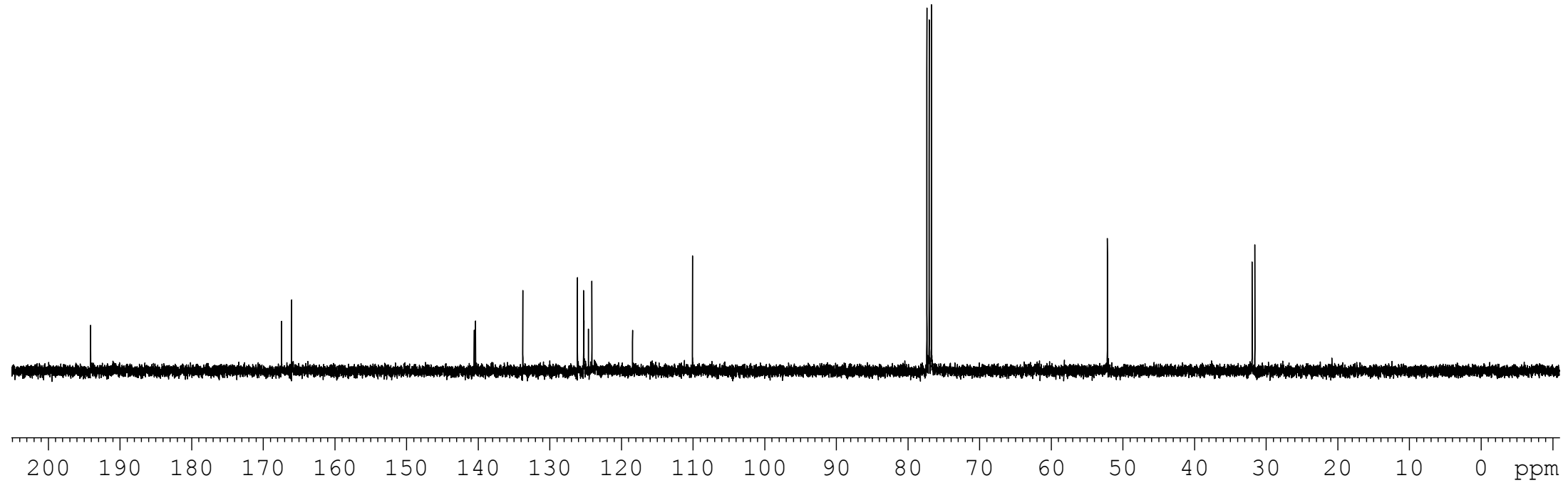




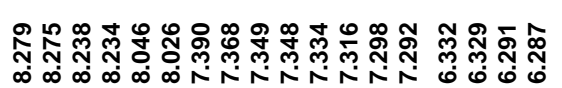

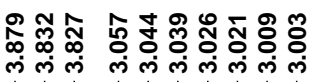

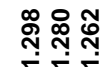

ฮั่

LVR-06200A

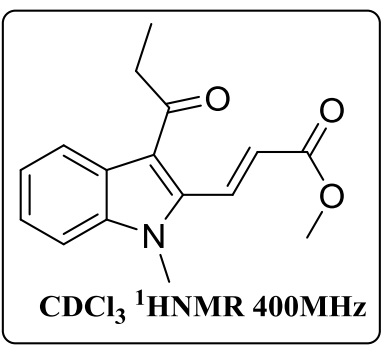

5 e

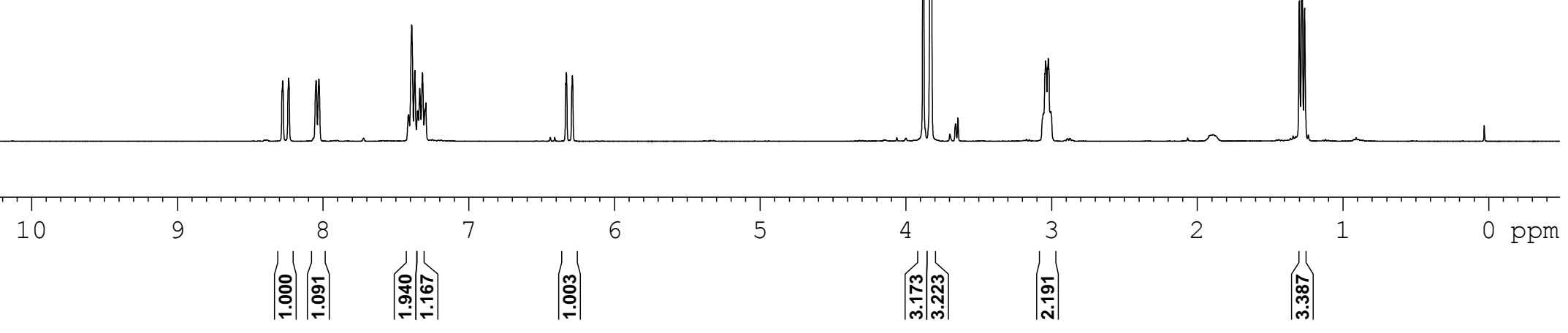

SI-64 


$\left.\right|^{\infty}$

LVR-06200A
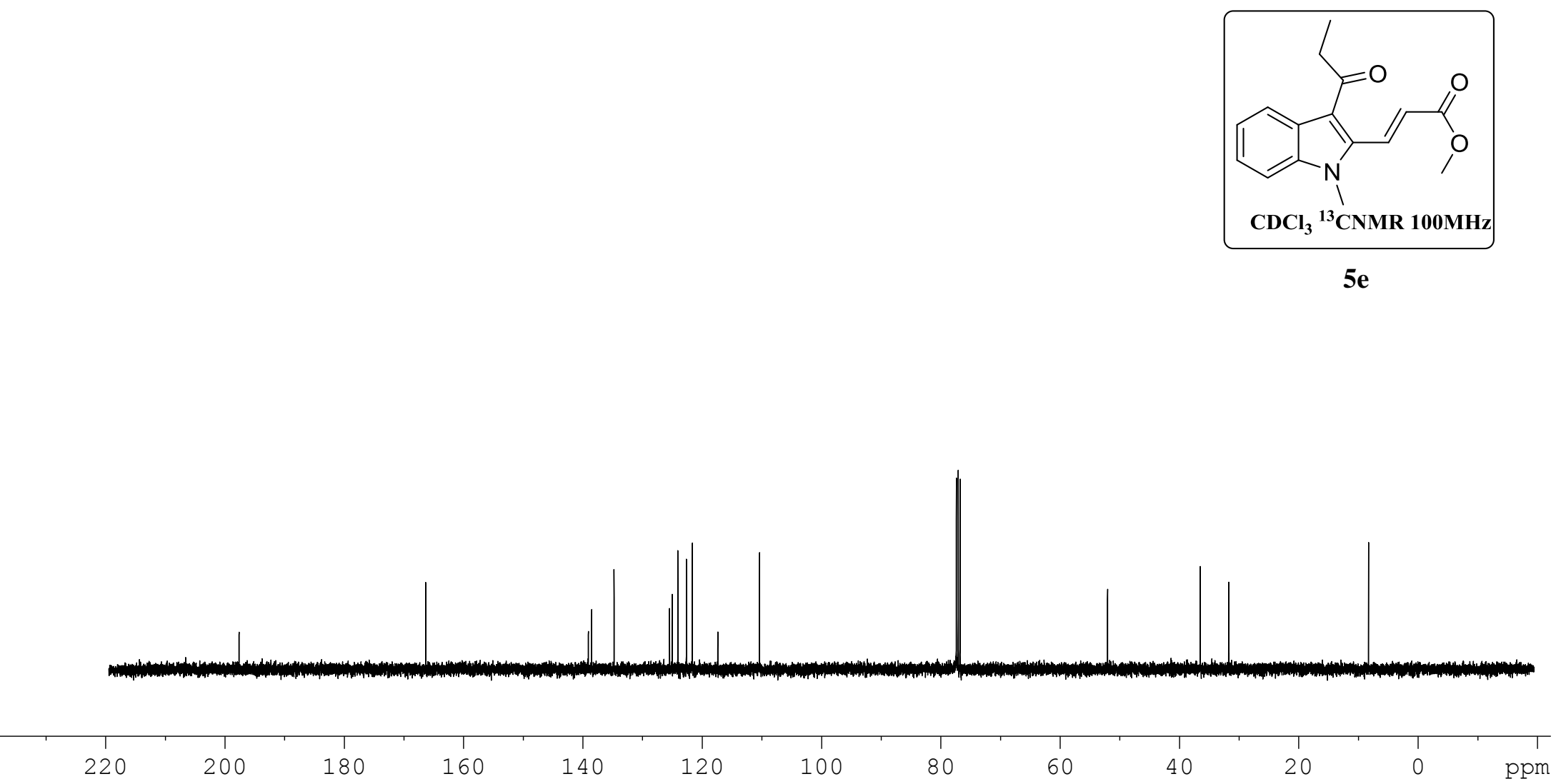


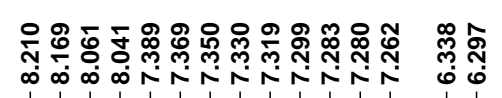

IV WW V

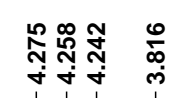

i
|

LVR-06193A

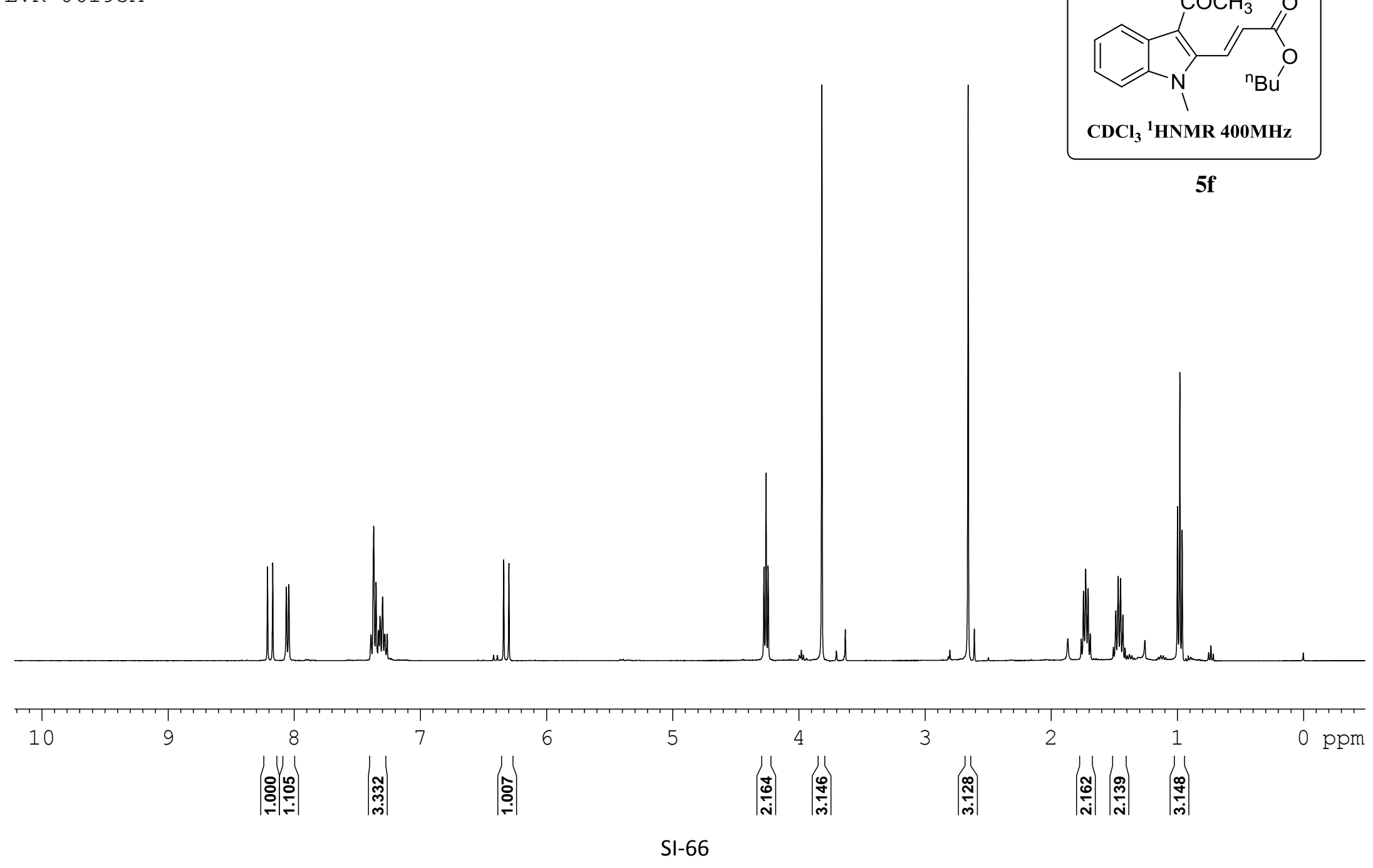




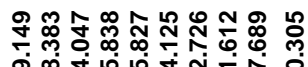

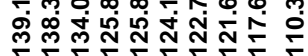

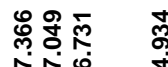

ト

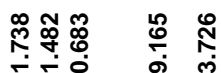

IVI/LI

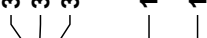

LVR-0 6193A
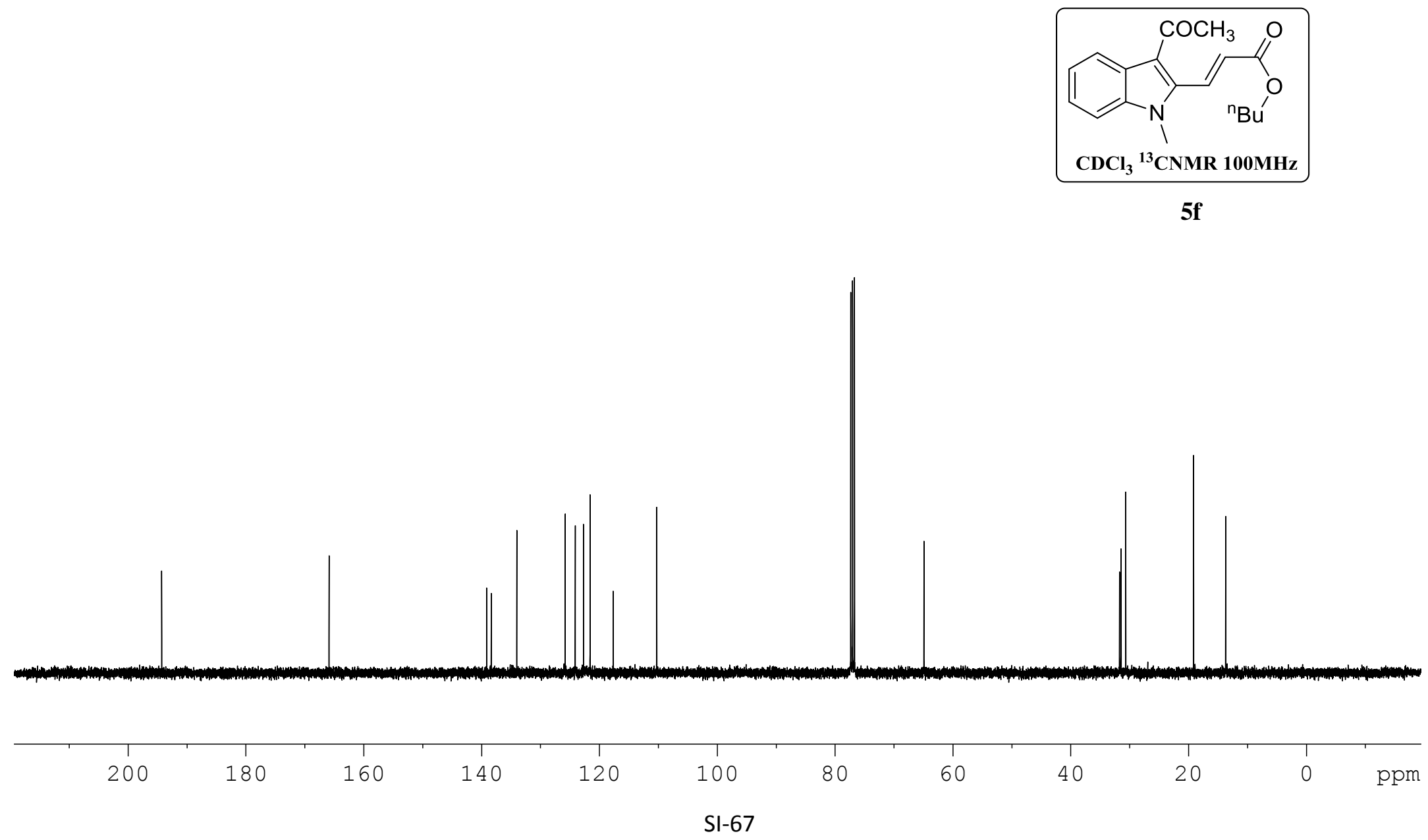


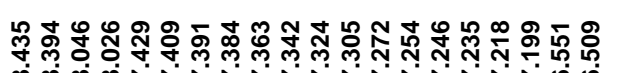

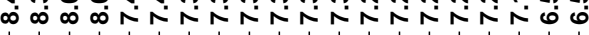

$\left.\right|_{\substack{\infty \\ \infty}} ^{\substack{\text { N } \\ \infty}}$

:

LVR-06194A

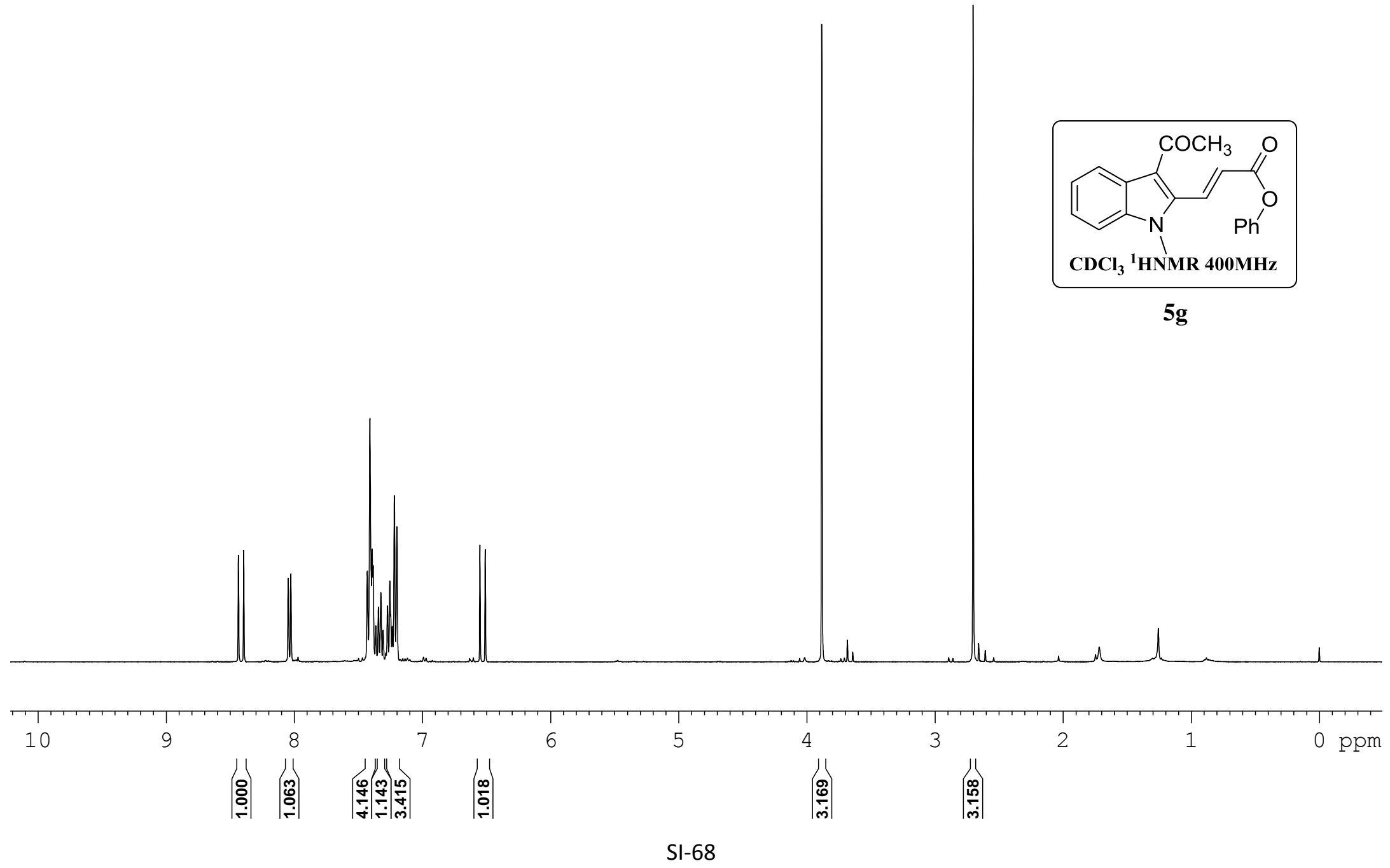




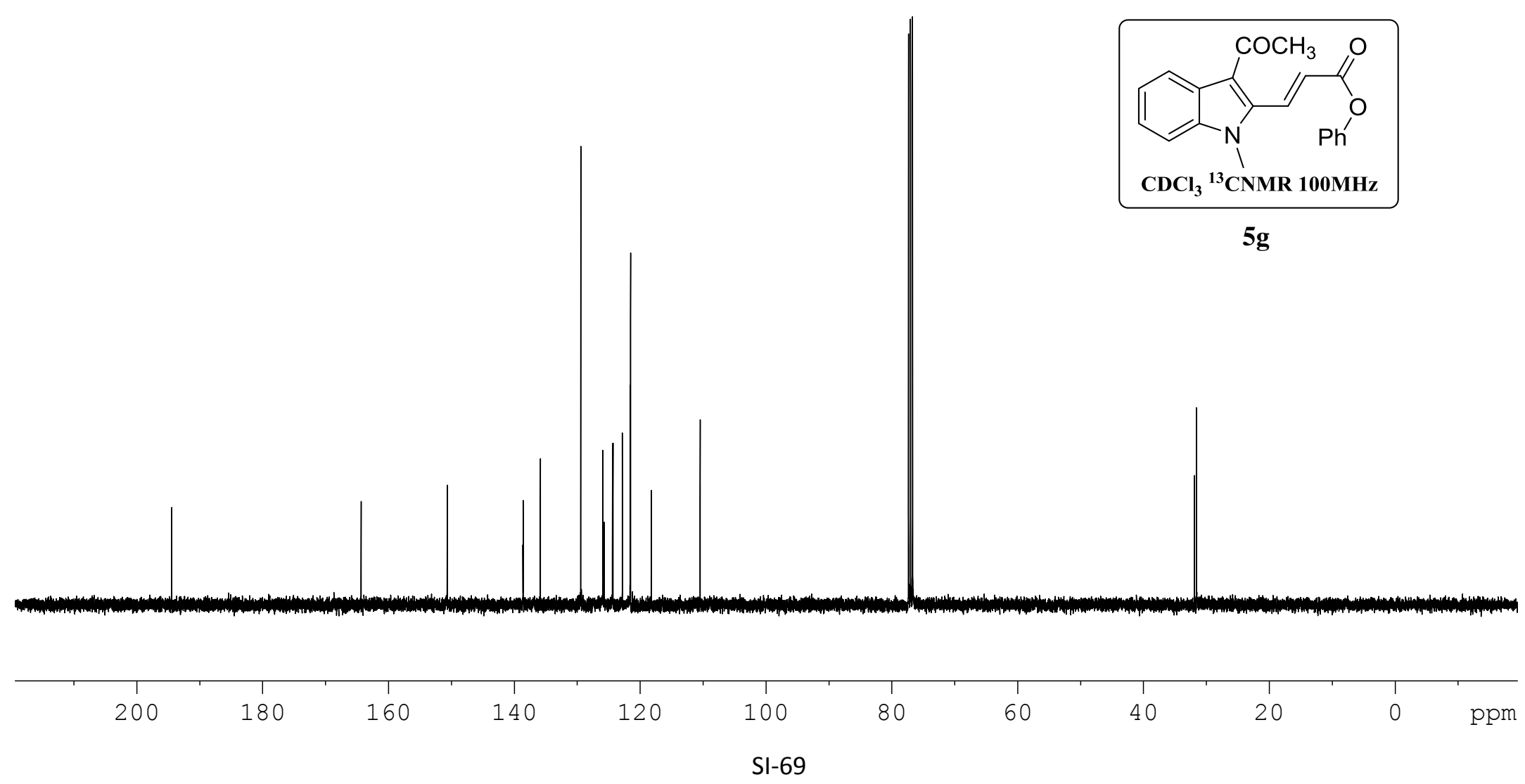



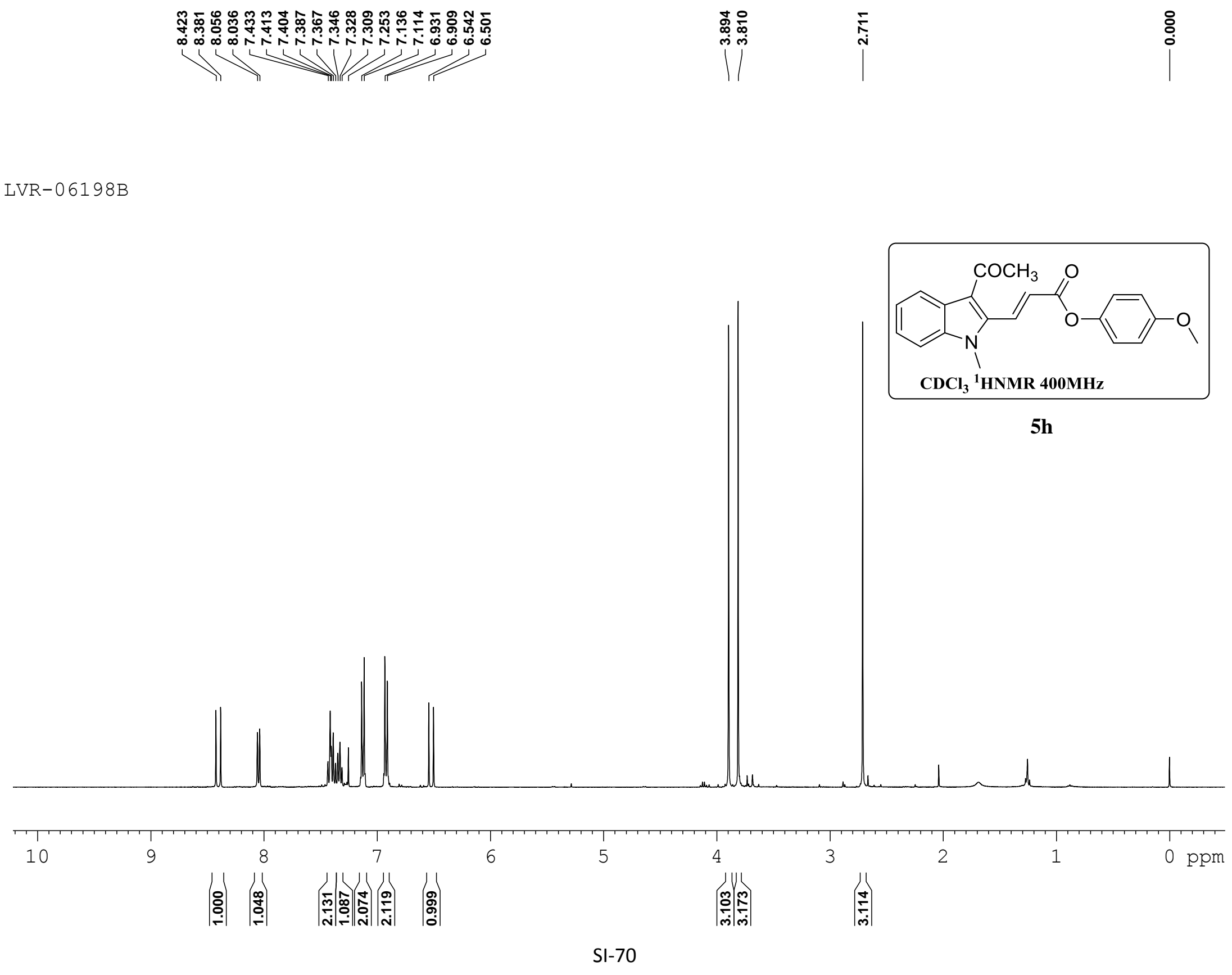


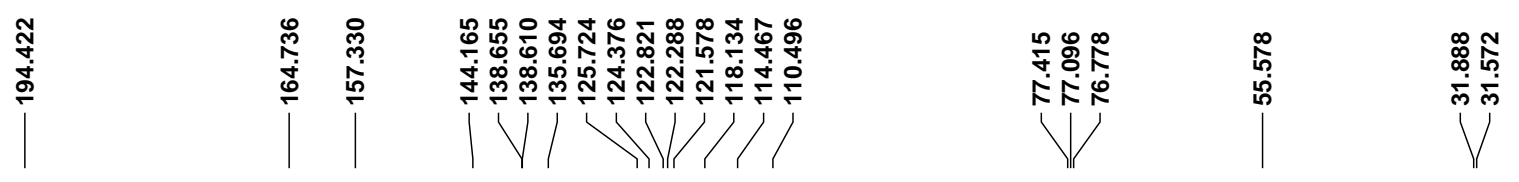

LVR-06198B

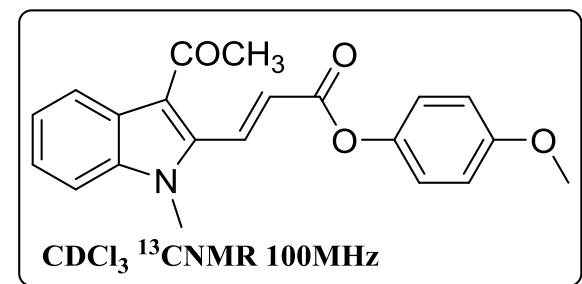

$5 h$

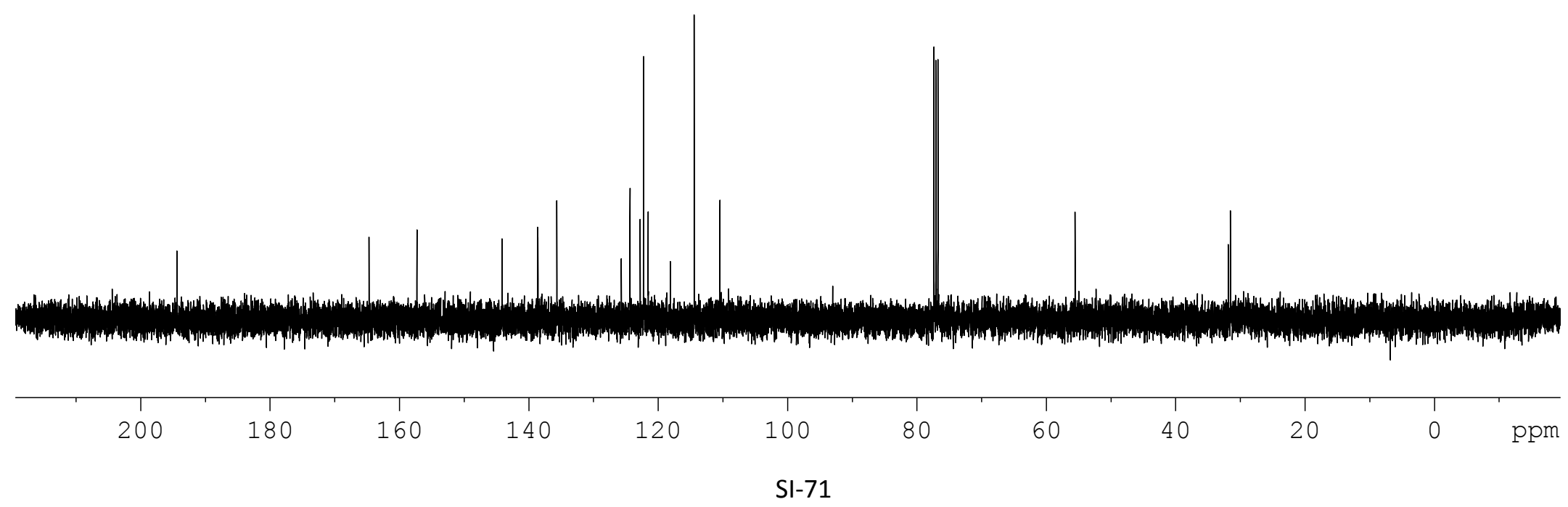




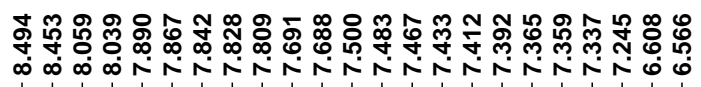

ֻั

$\stackrel{\text { ลิ }}{\text { งิ }}$

๕

LVR-06198A

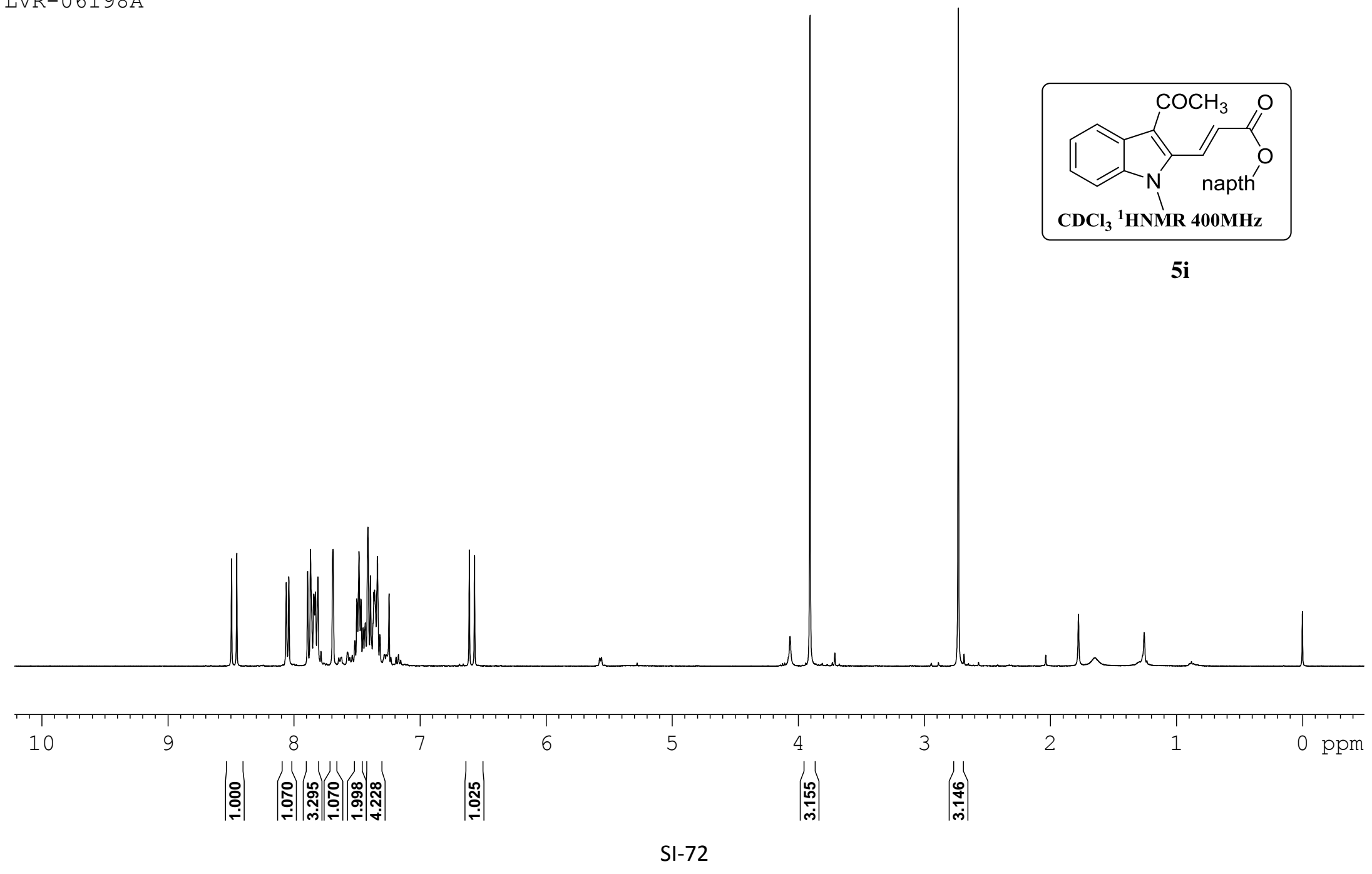




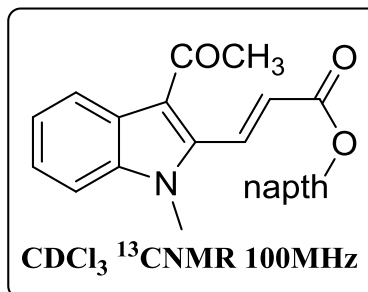

$5 \mathbf{i}$

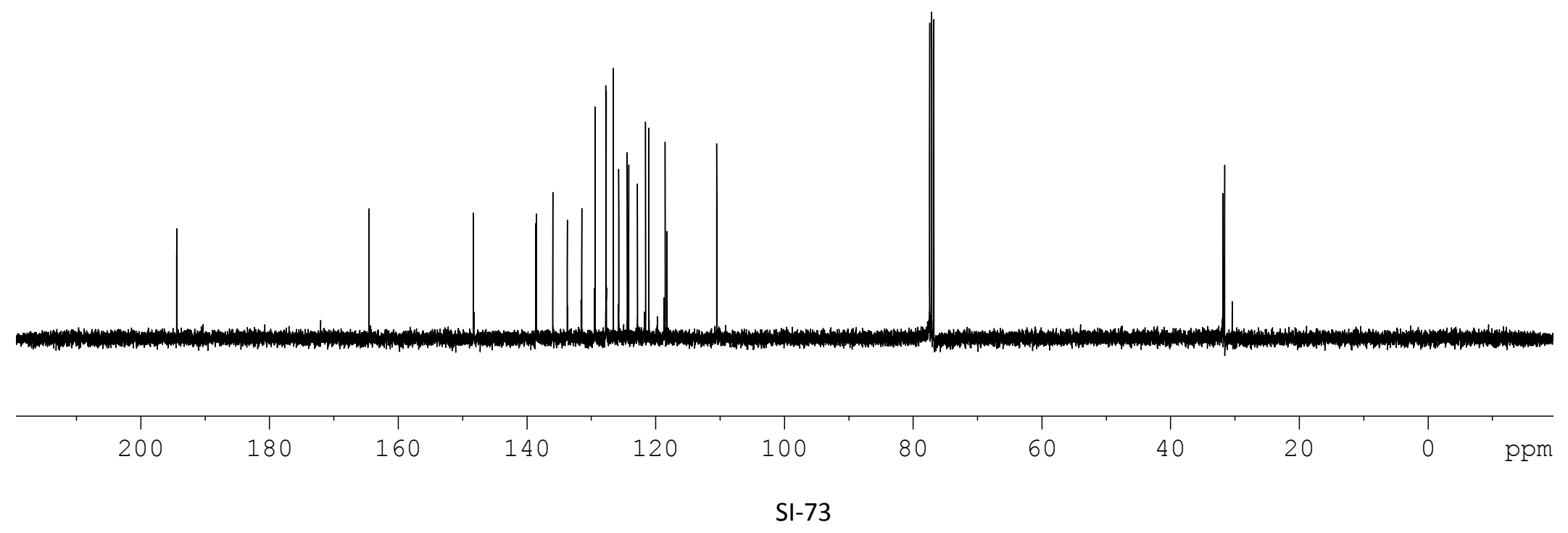


Lanke, Bettadapur, and Prabhu/Org Chem/IISc

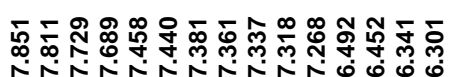

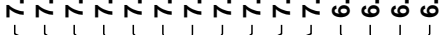

VIIII

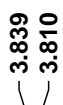

ฐั๊

ō

LVR-06114B

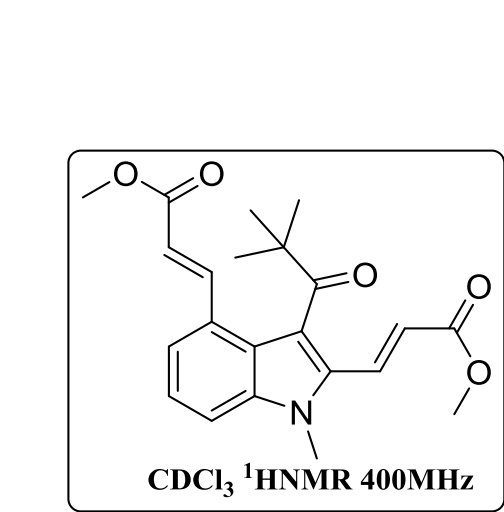

6a

V

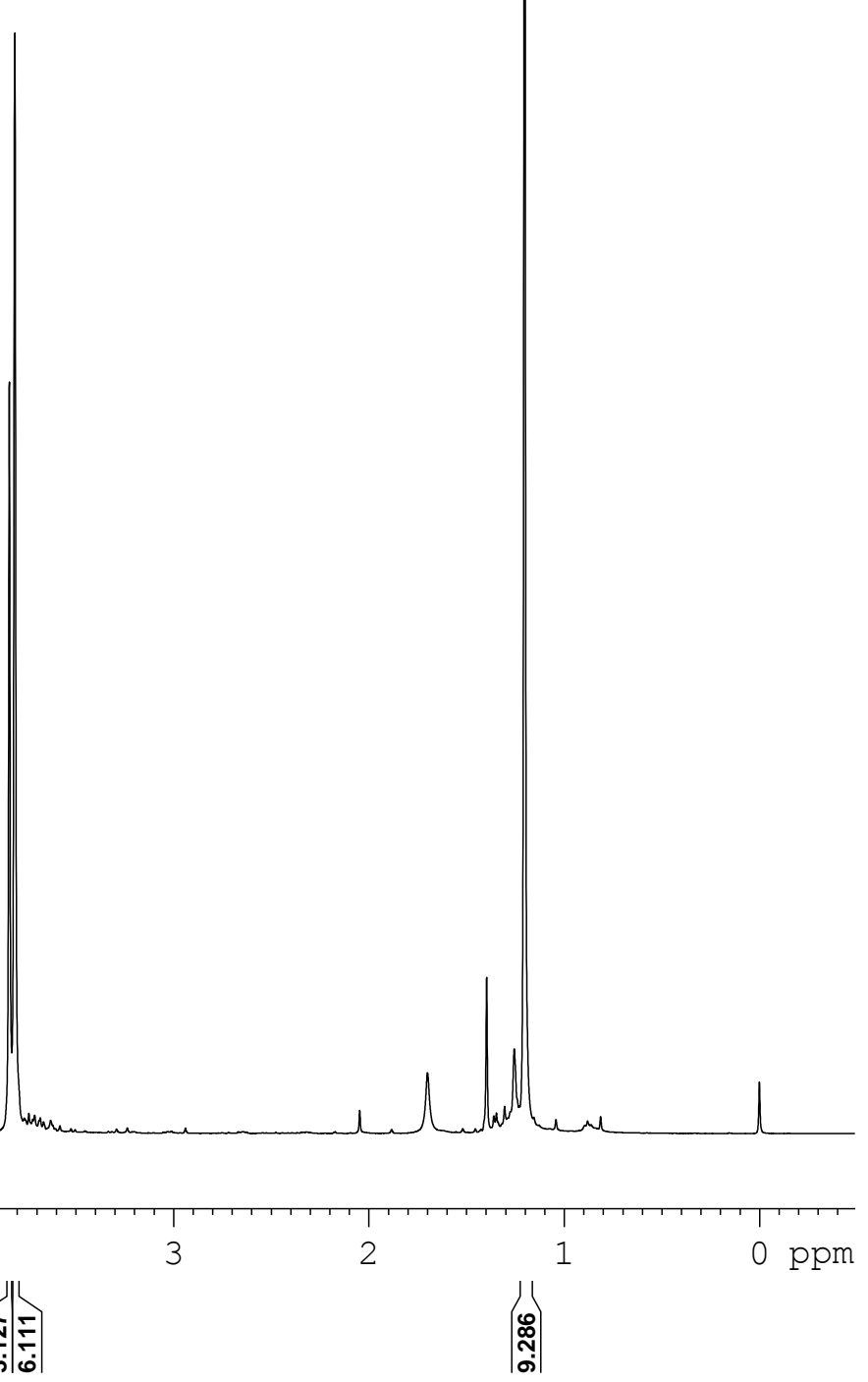

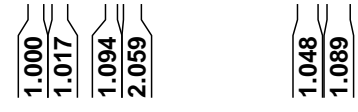

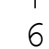

5

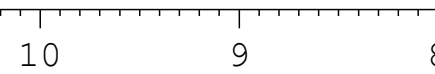

बَ̄ 


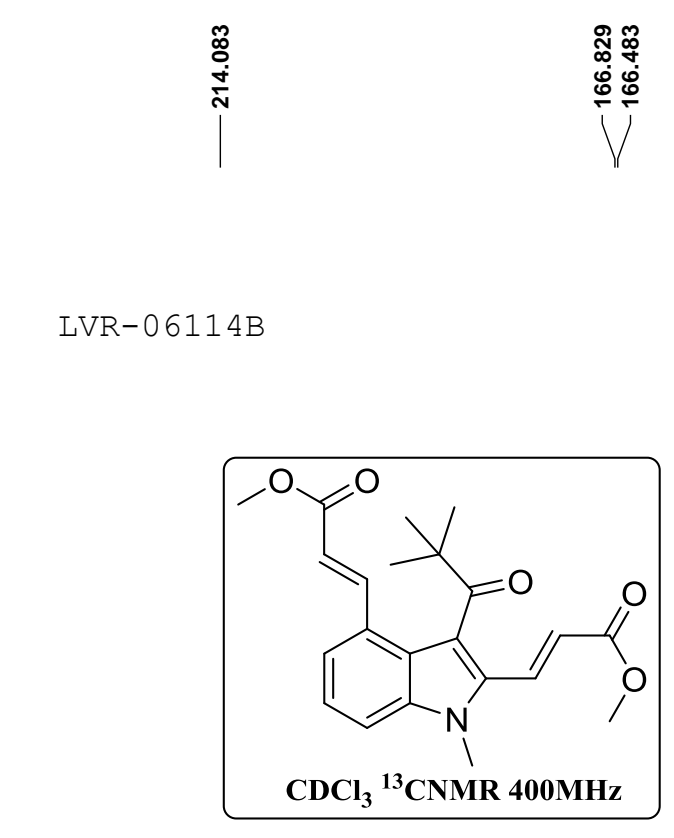

$6 a$

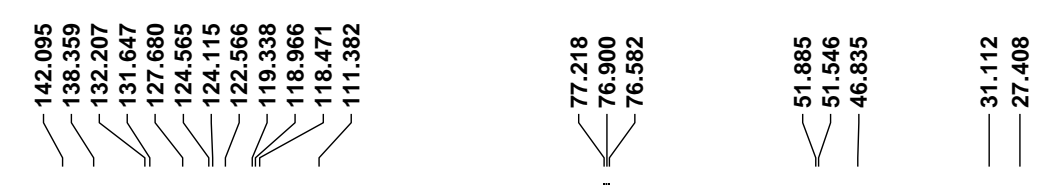

LVR-06114B

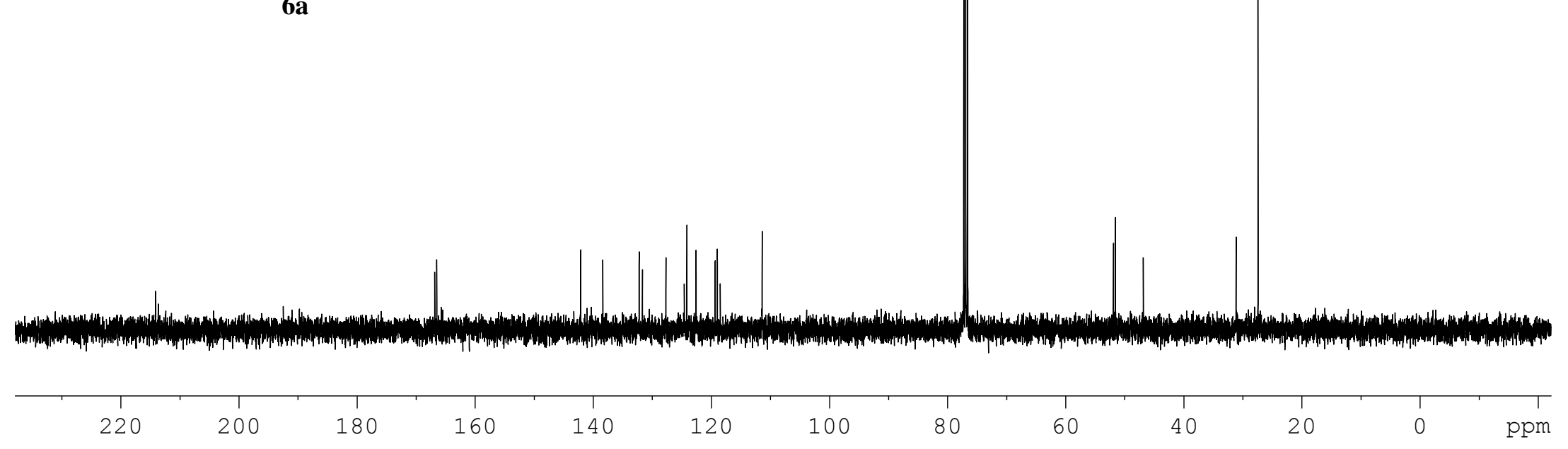



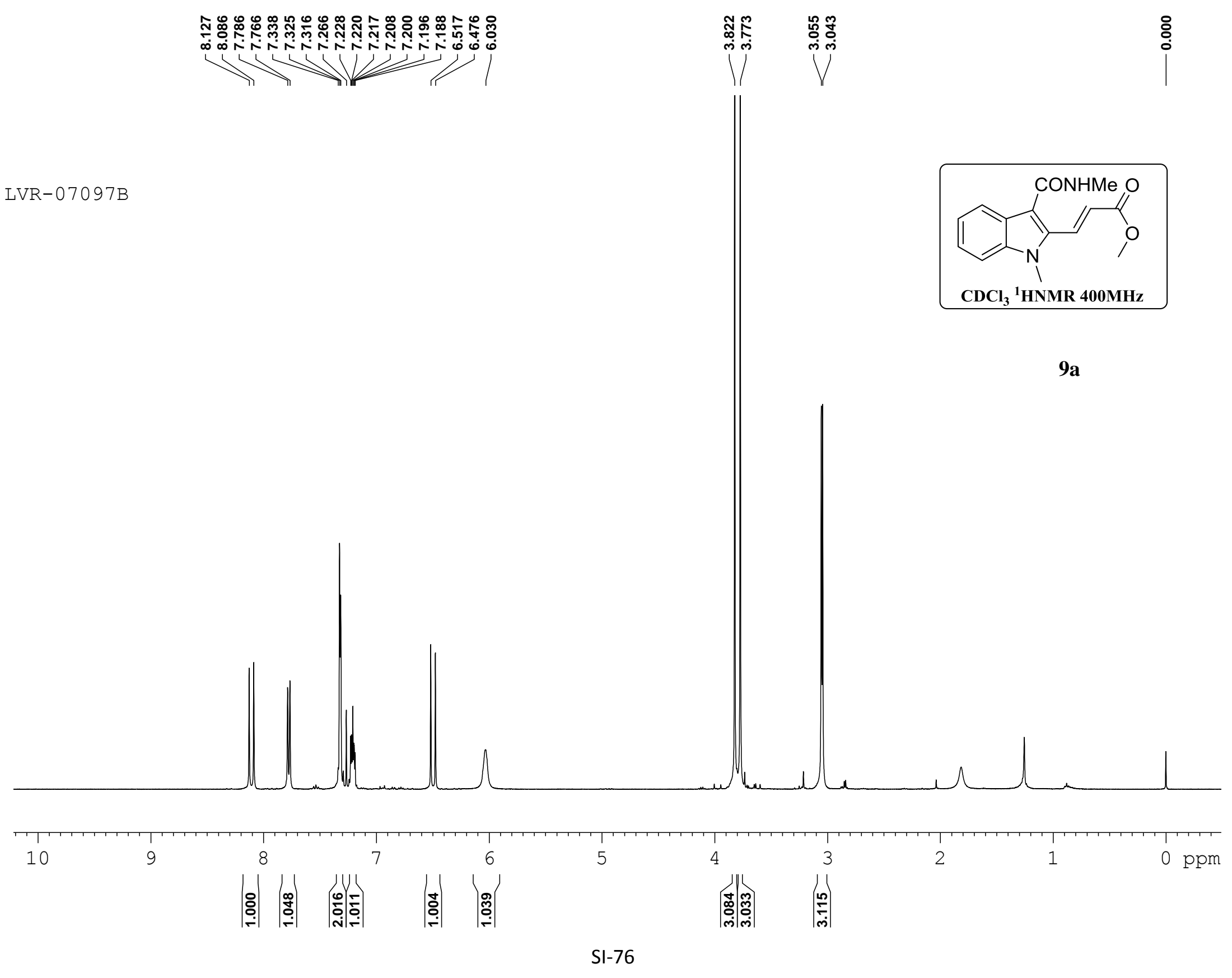


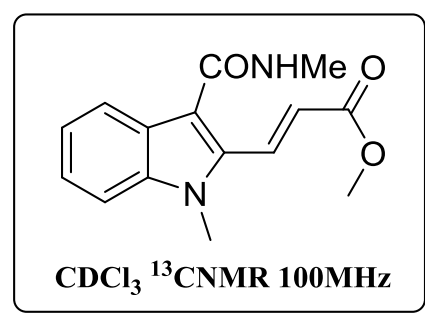

9a

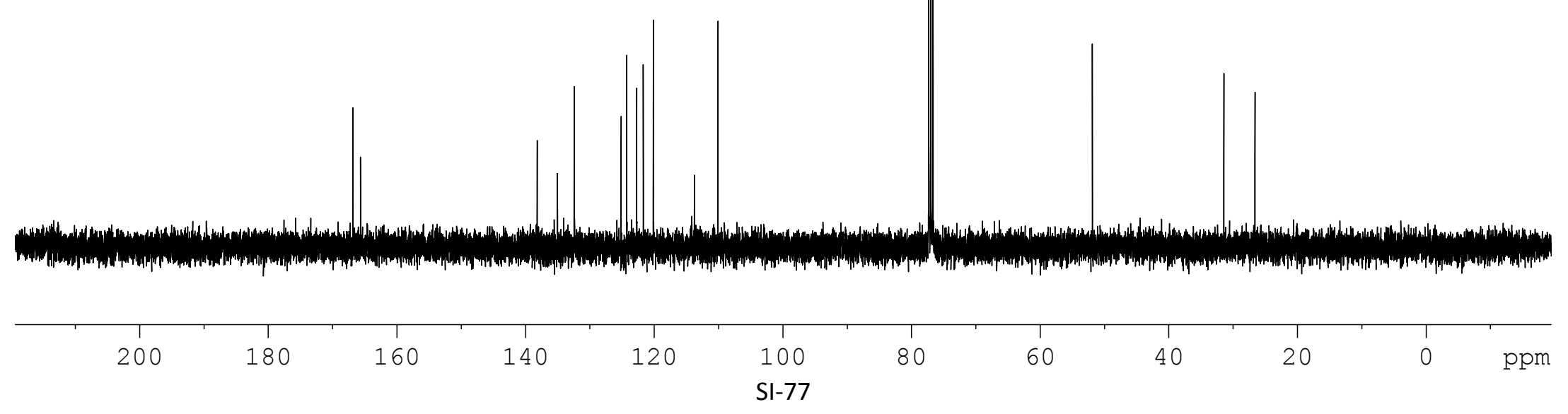



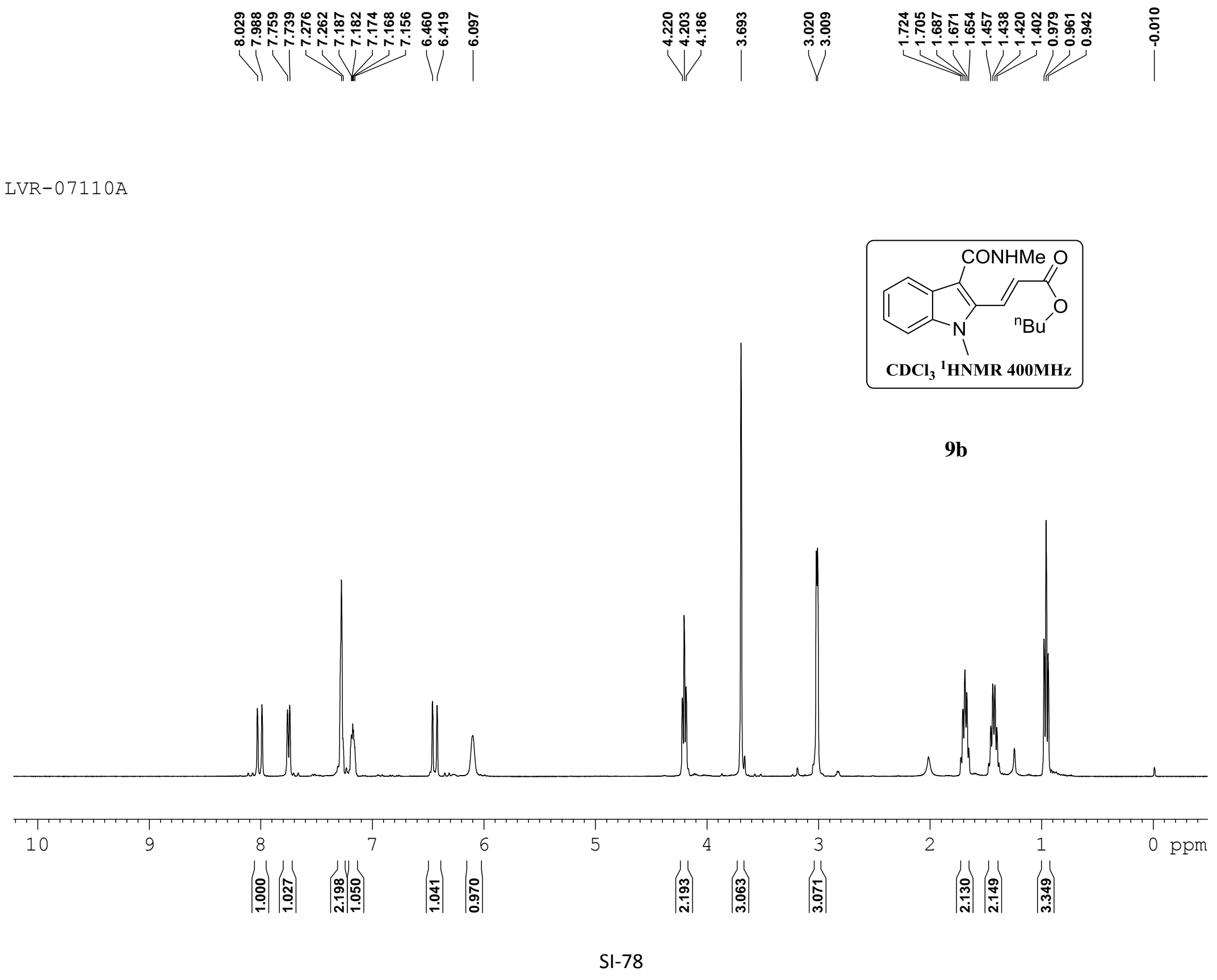


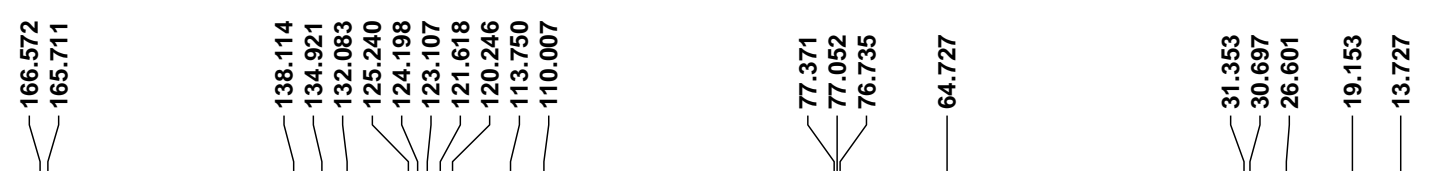

LVR-07110A

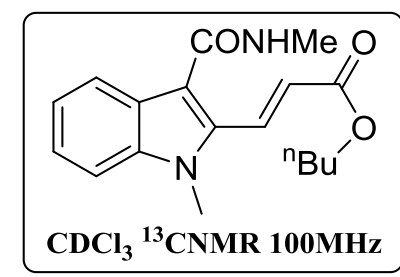

9b

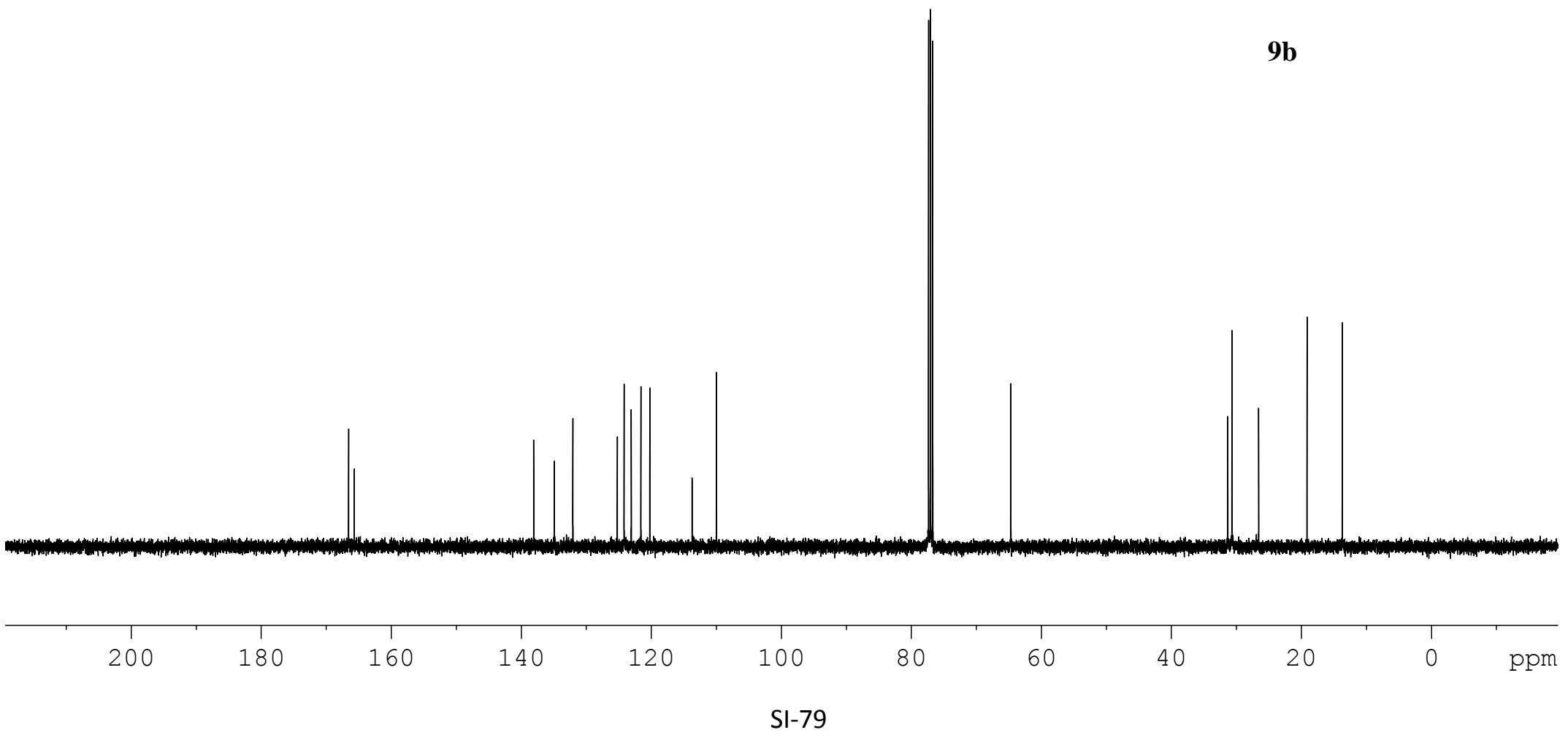




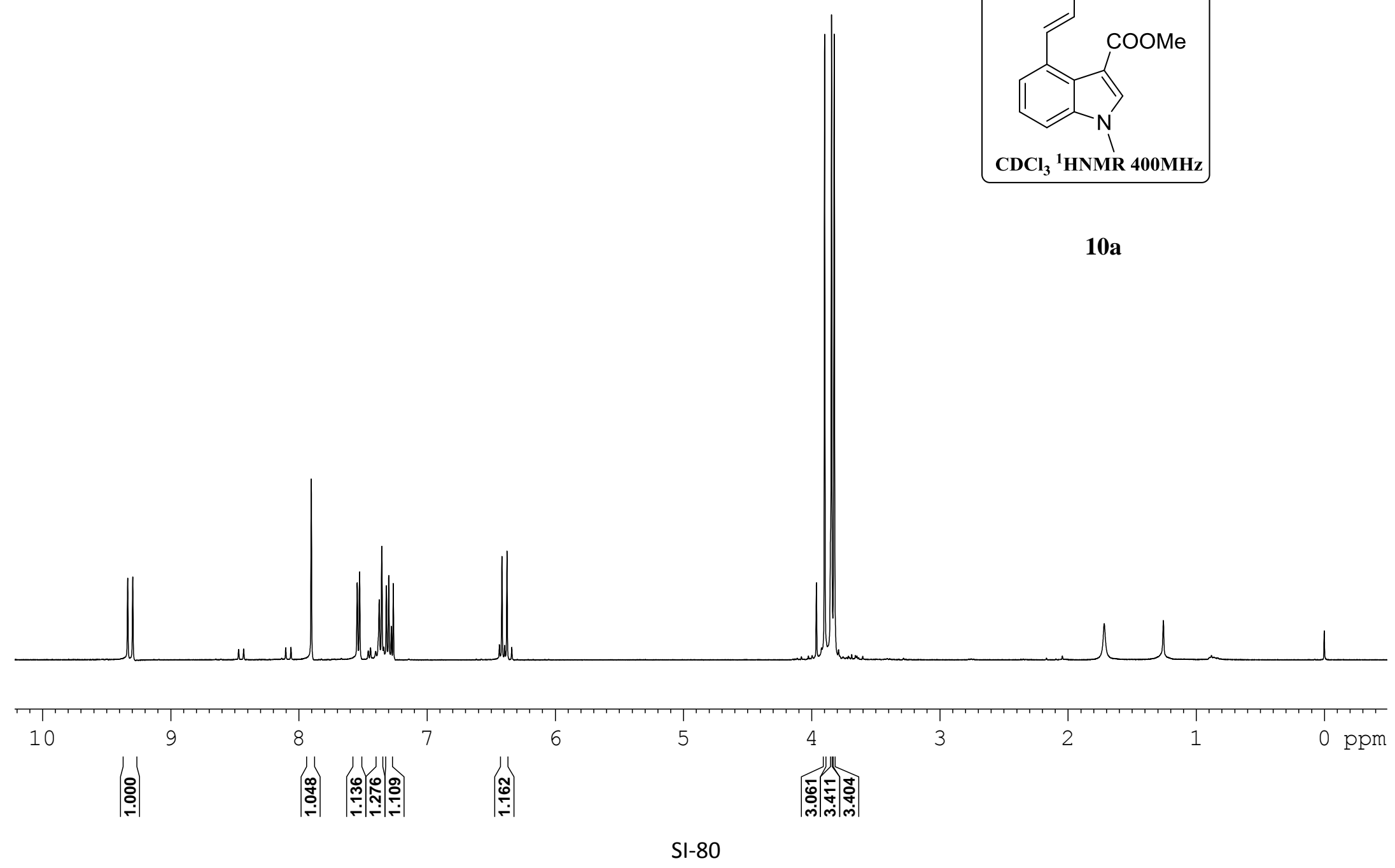




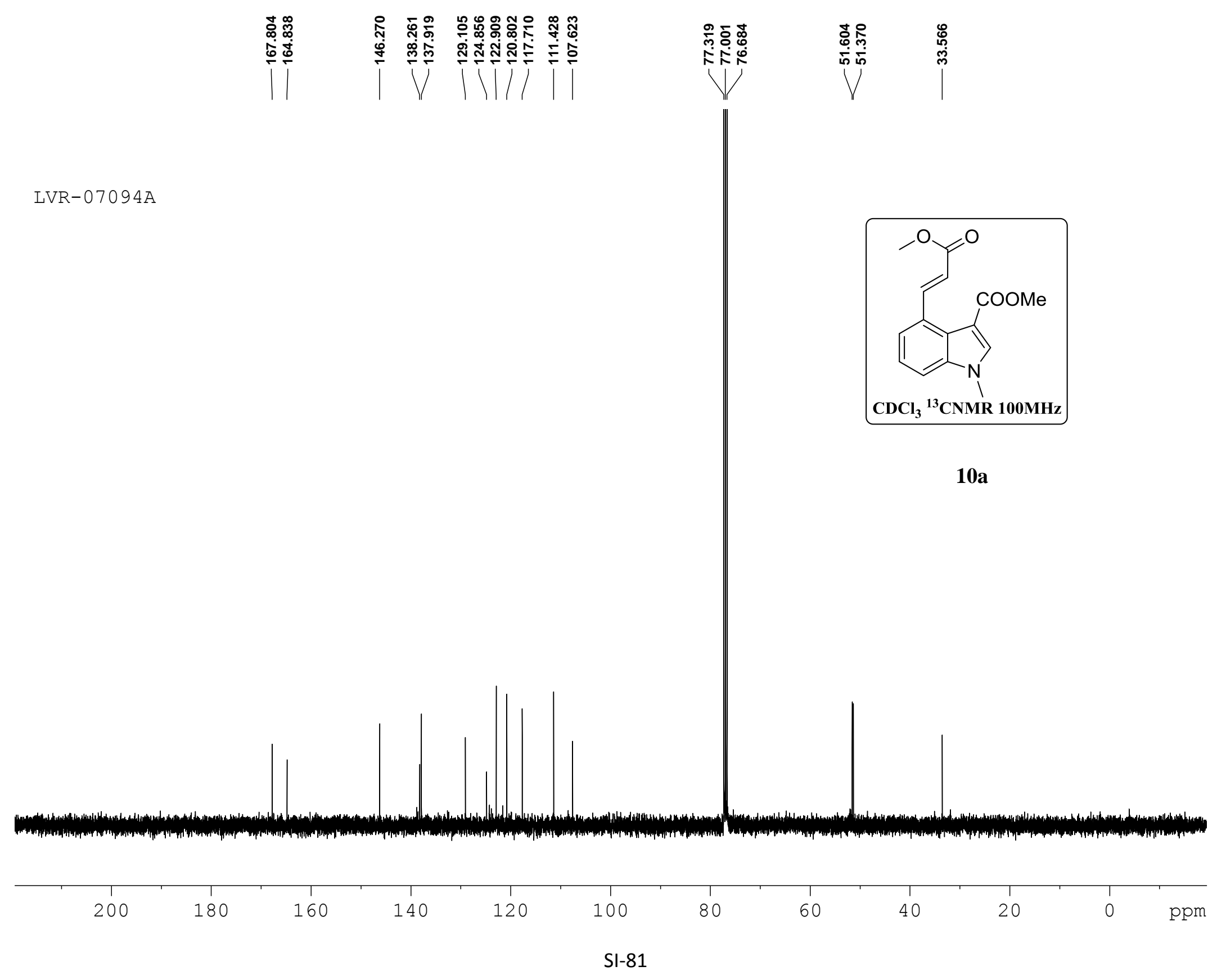




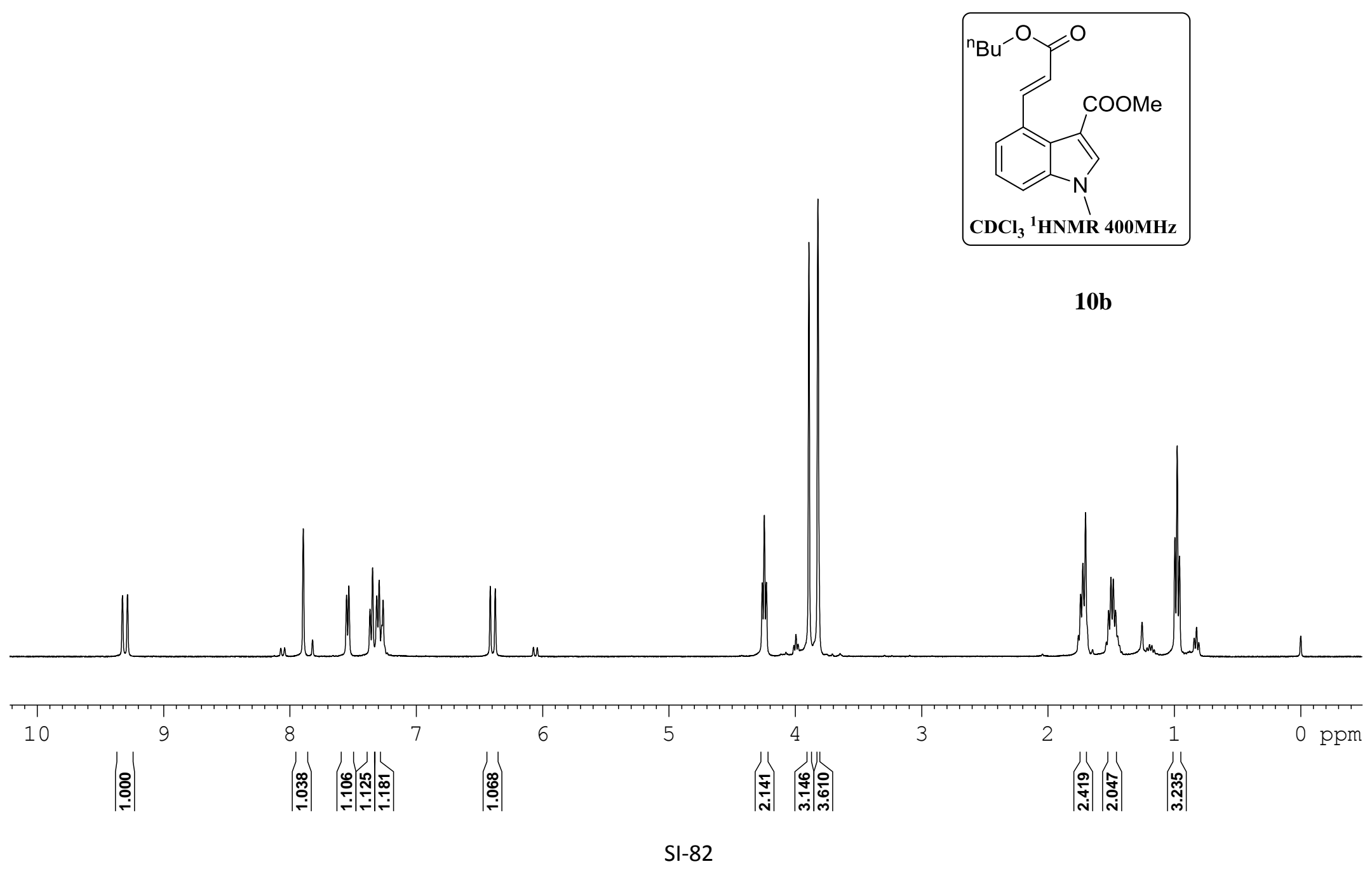



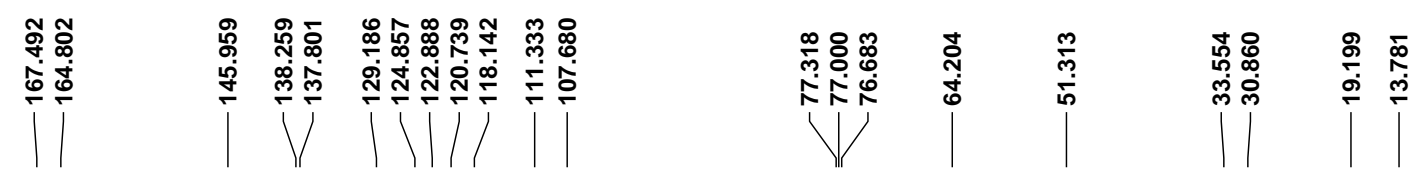

JVR-07108A

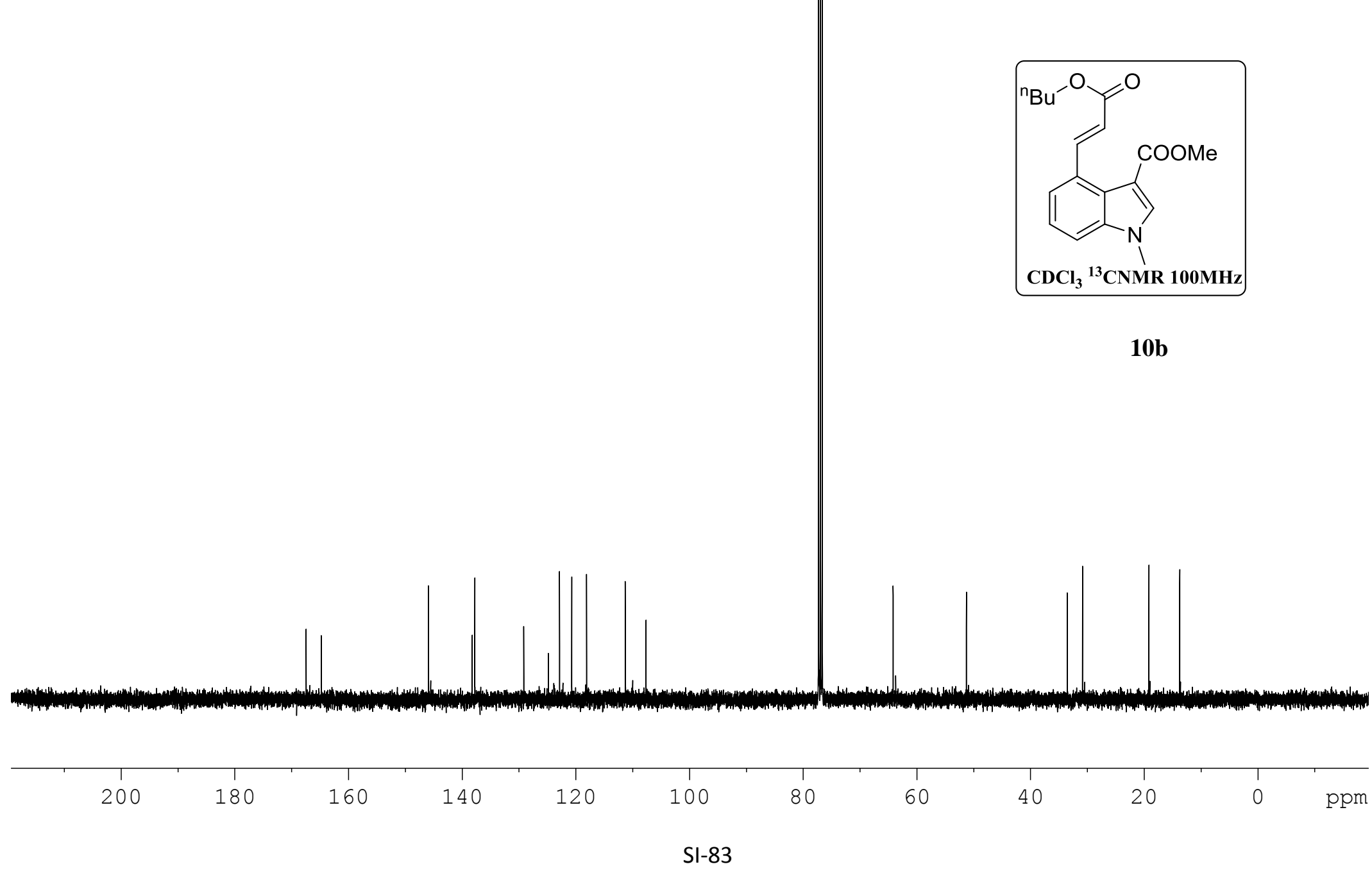


Starting Material - 1a

Deuterating agent $-\mathrm{D}_{2} \mathrm{O}$

C-4 deuteration $-70 \%$

C-2 deuteration $-3 \%$

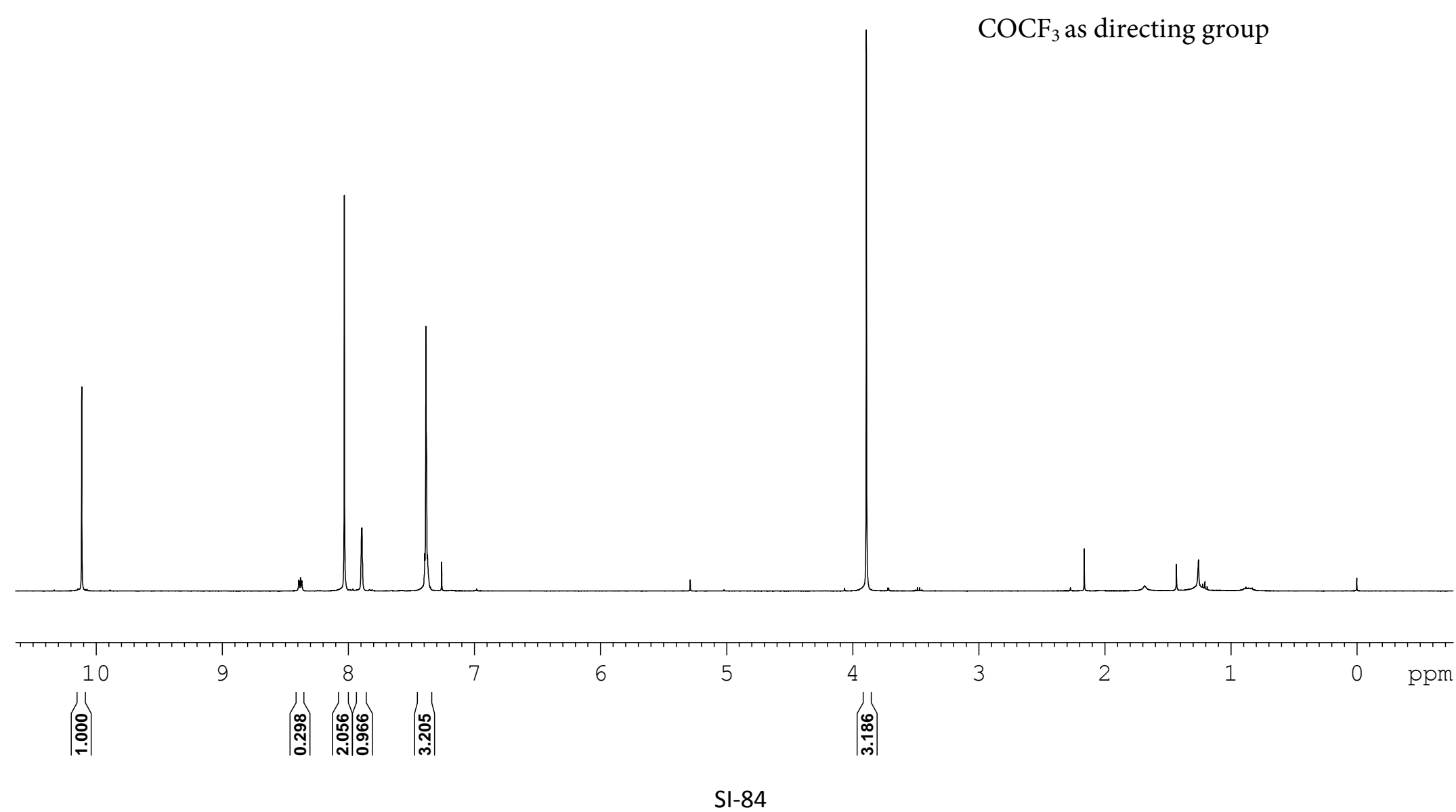


Lanke, Bettadapur, and Prabhu/Org Chem/IISc

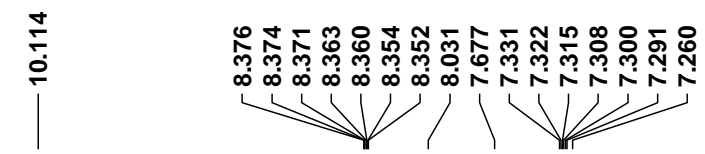

LVR-07179B

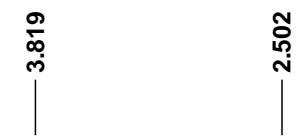

\section{Deuterium Incorporation studies:}

Internal Standard - terephthaldehyde ( 0.5 equiv)

Starting Material - 2a

Deuterating agent $-\mathrm{D}_{2} \mathrm{O}$

C-4 deuteration $-78 \%$

C-2 deuteration $-77 \%$

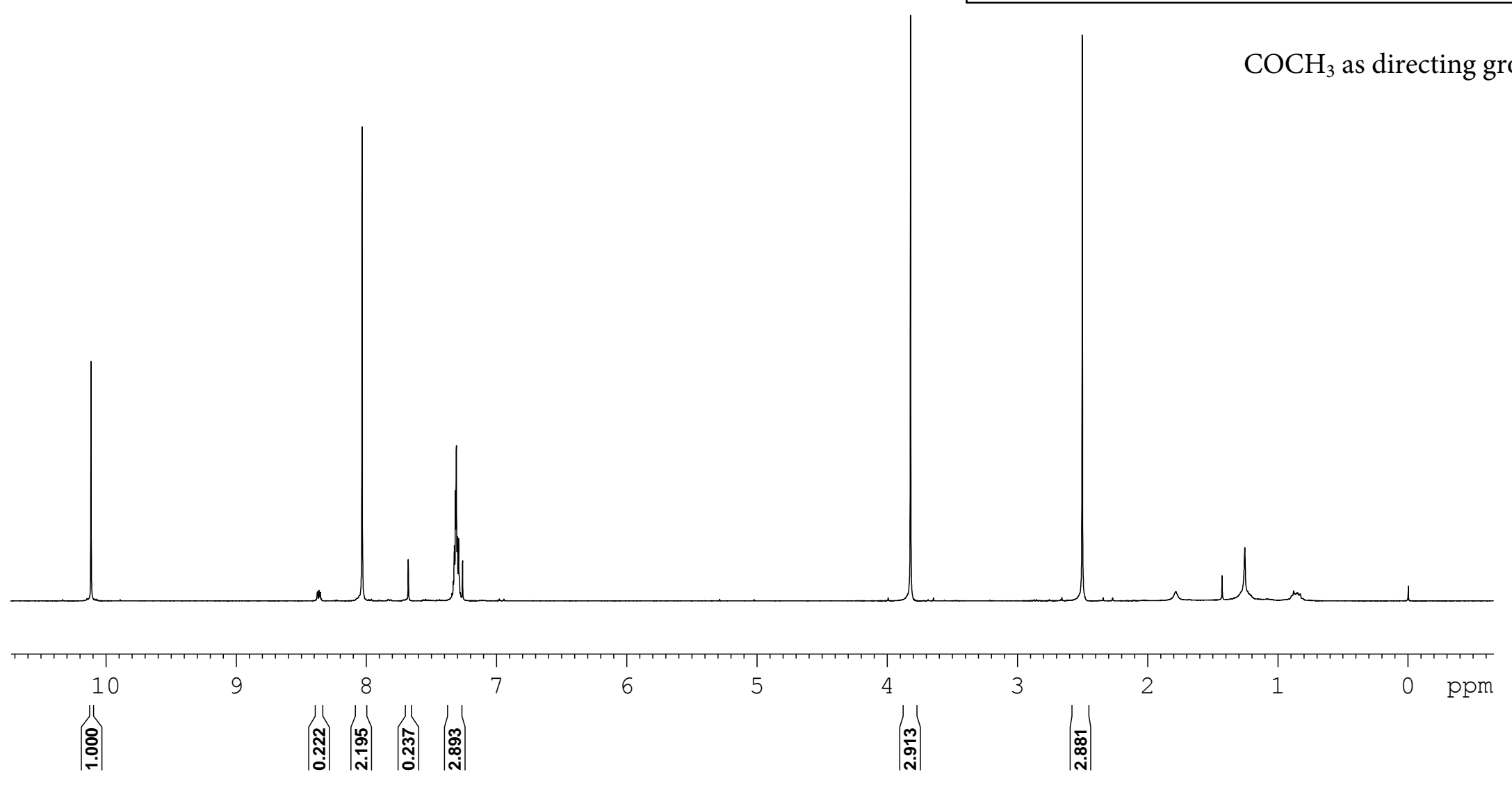


Lanke, Bettadapur, and Prabhu/Org Chem/IISc

立

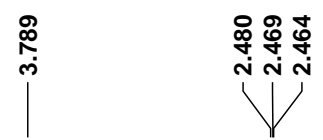

웅

LVR-07171B

\section{Deuterium Incorporation studies:}

Internal Standard - terephthaldehyde ( 0.5 equiv)

Starting Material - 2a

Deuterating agent $-\mathrm{AcOD}$

C-4 deuteration $-60 \%$

C-2 deuteration $-60 \%$

$\alpha$ deuteration $-30 \%$

$\mathrm{COCH}_{3}$ as directing group

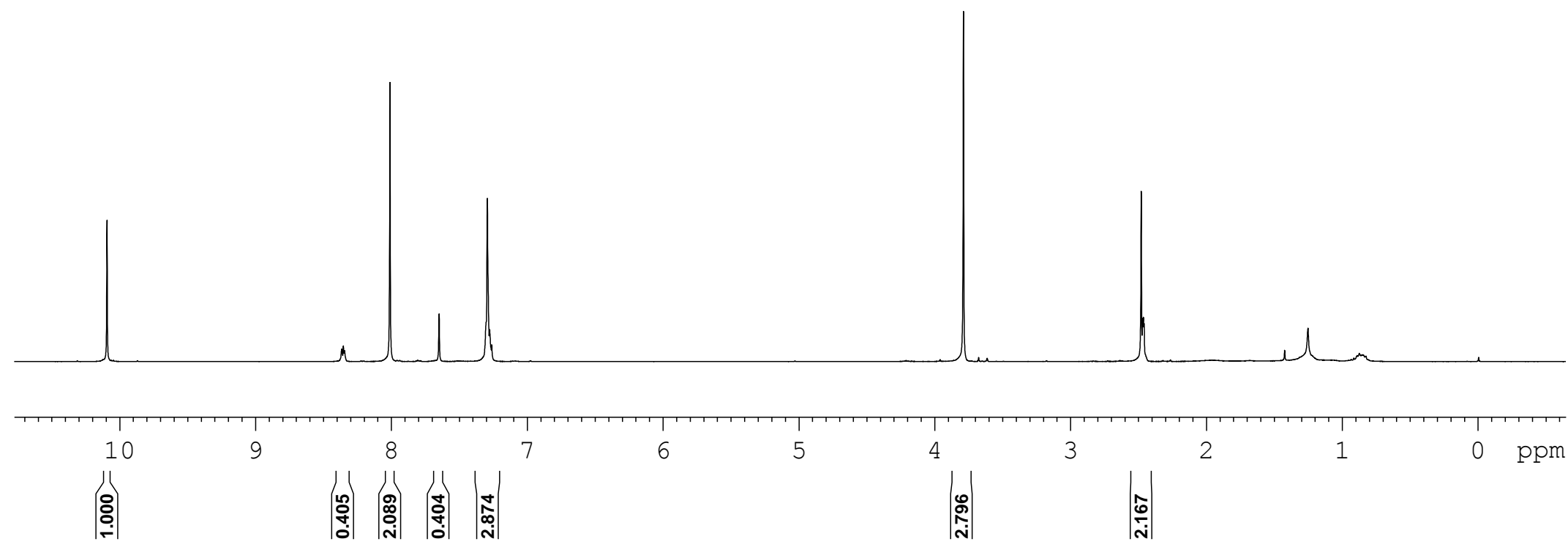


Lanke, Bettadapur, and Prabhu/Org Chem/IISc

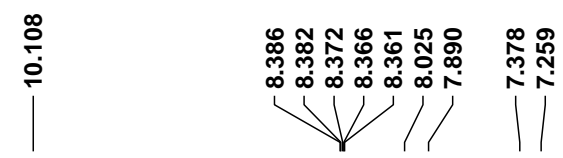

\section{Deuterium Incorporation studies:}

Internal Standard - terephthaldehyde ( 0.5 equiv)

Starting Material - 1a

Deuterating agent $-\mathrm{AcOD}$

C-4 deuteration $-66 \%$

C-2 deuteration $-15 \%$

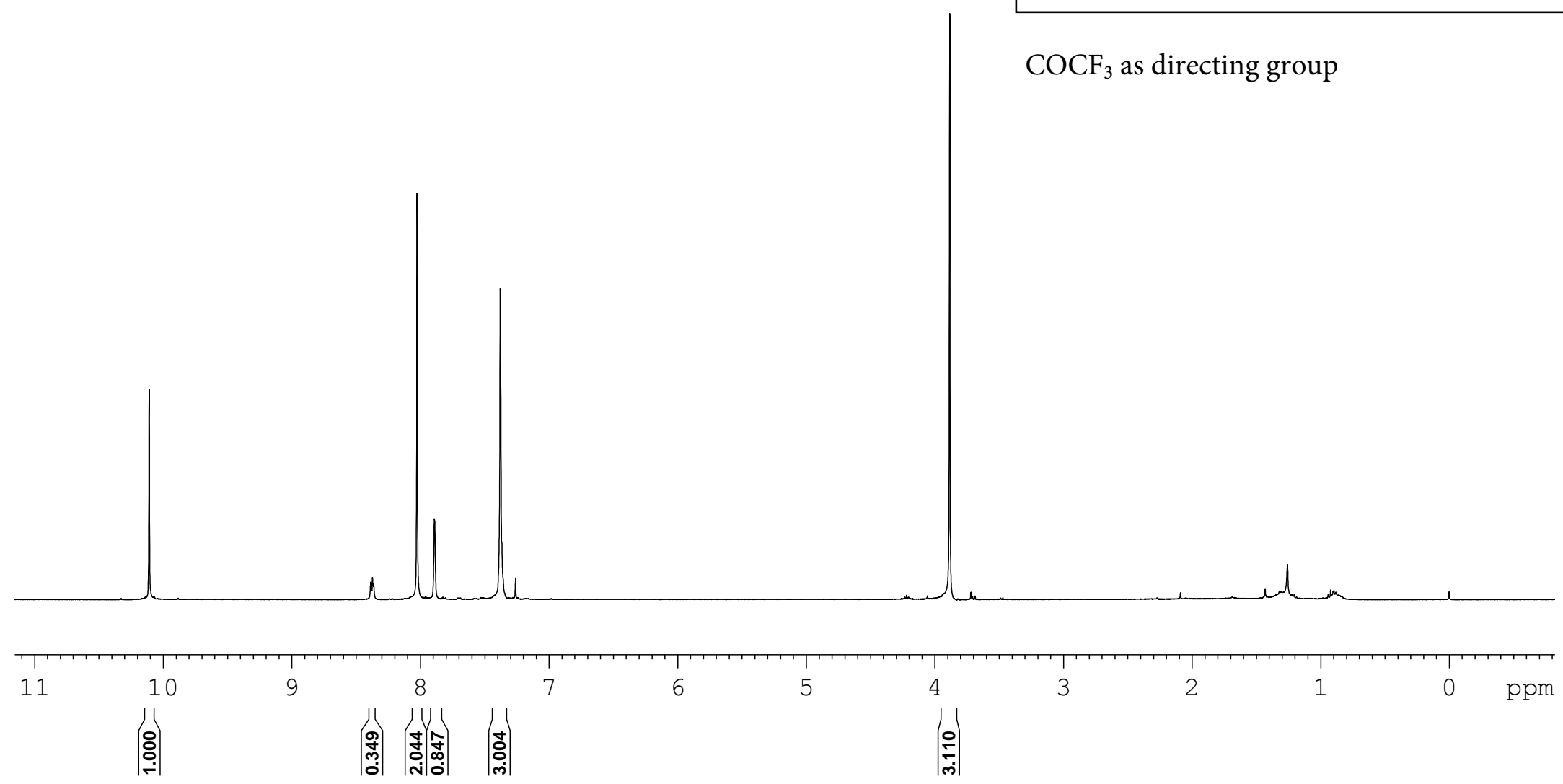




\section{Crystal Structures of Product 4a and 5a (cif file attached as separate} file)

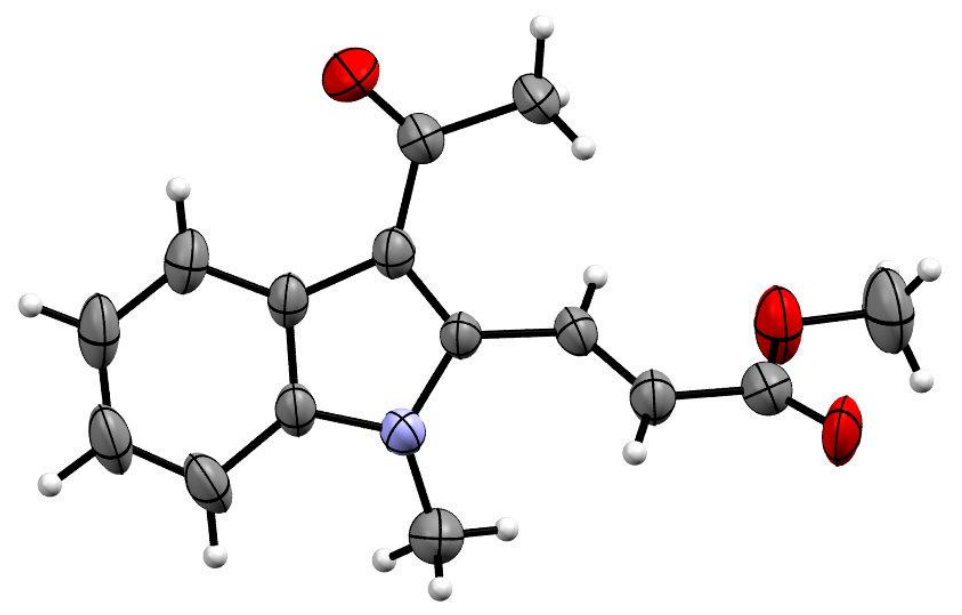

$5 a$

CCDC Number - 1438625

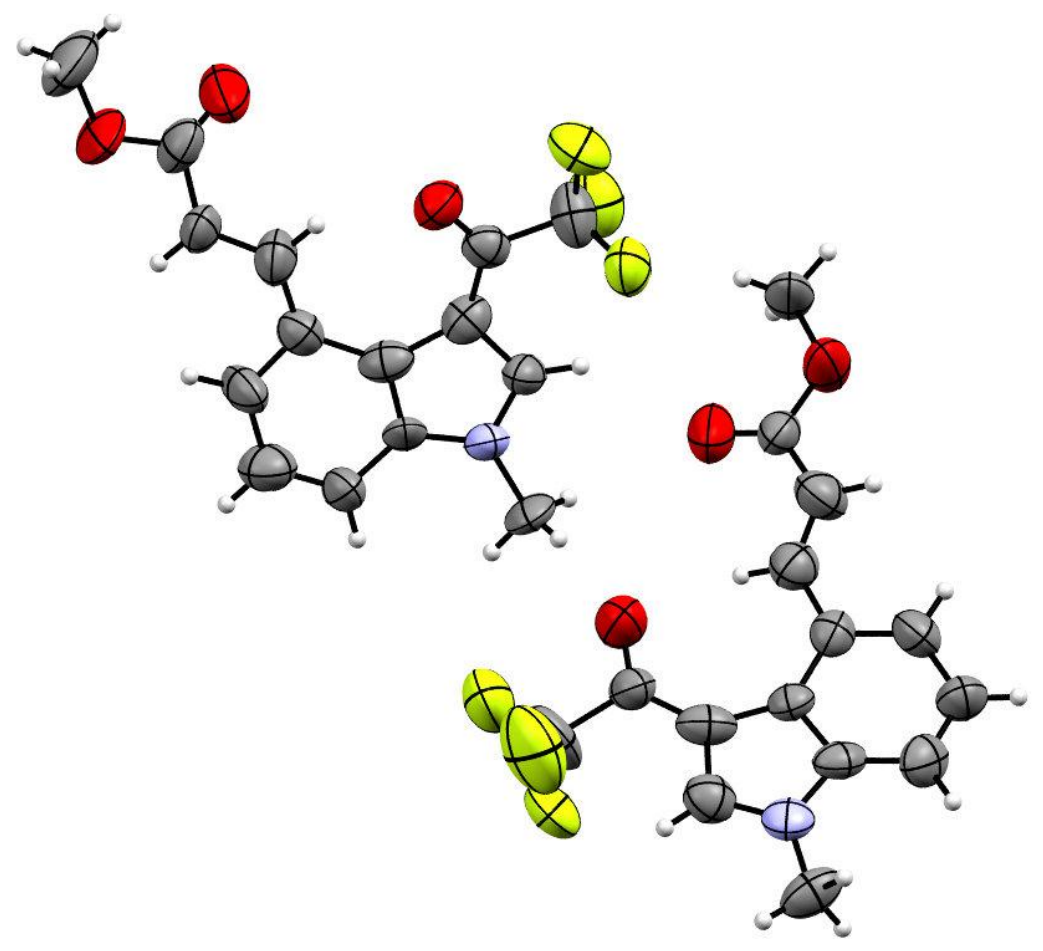

4a

CCDC Number - 1438626 